\title{
Trans Influence of Ligands on the Oxidation of Gold(I) Complexes
}

Yangyang Yang,${ }^{\dagger}$ Lukas Eberle,${ }^{\dagger}$ Florian F. Mulks, ${ }^{\dagger}$ Jonas F. Wunsch,${ }^{\dagger}$ Marc Zimmer,${ }^{\dagger}$ Frank Rominger,${ }^{\dagger}$ Matthias Rudolph ${ }^{\dagger}$ and A. Stephen K. Hashmi*,†,

*Organisch-Chemisches Institut, Universitat Heidelberg, Im Neuenheimer Feld 270, 69120 Heidelberg, Germany

*Chemistry Department, Faculty of Science, King Abdulaziz University (KAU), Jeddah 21589, Saudi Arabia

*Email: hashmi@hashmi.de

\section{Table of Contents}

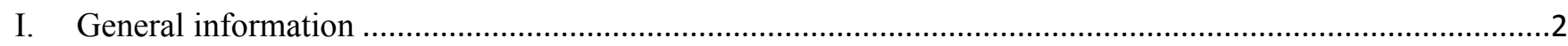

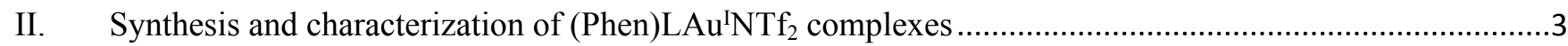

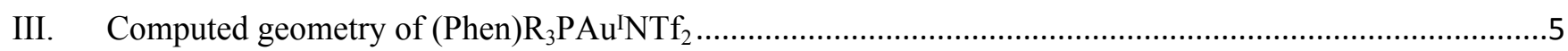

IV. Oxidative addition to asymmetric tri-coordinate gold(I) complexes ....................................................9

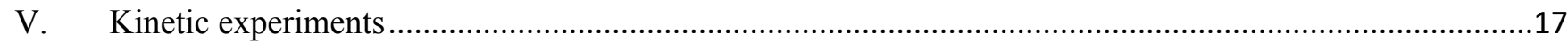

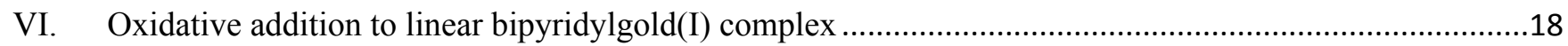

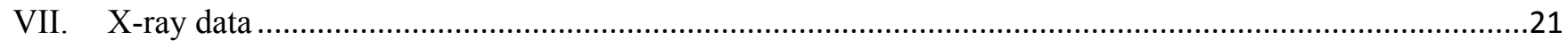

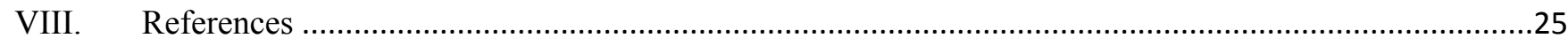

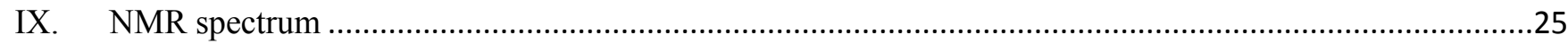




\section{General information}

Chemicals were purchased from commercial suppliers and used as delivered. Dry solvents were dispensed from solvent purification system MB SPS-800. Oxygen-free solvents were employed. Unless otherwise stated, all reactions and manipulations were carried out under an atmosphere of dry $\mathrm{N}_{2}$ using standard Schlenk techniques or in a glovebox under an inert atmosphere. ${ }^{1} \mathrm{H},{ }^{13} \mathrm{C}\left\{{ }^{1} \mathrm{H}\right\}$, and ${ }^{19} \mathrm{~F}$ NMR spectra were recorded on a Avance III, Avance 400, Avance-III-300, Avance DRX-300, Avance 500 or Avance 600. Chemical shifts are expressed as parts per million (ppm, $\delta$ ) downfield of tetramethylsilane (TMS) and are referenced to $\mathrm{CDCl}_{3}$ (7.26 / $\left.77.32 \mathrm{ppm}\right), \mathrm{CD}_{3} \mathrm{CN}(1.94 / 1.32,118.26 \mathrm{ppm}), \mathrm{CD}_{2} \mathrm{Cl}_{2}(5.32$ / $53.84 \mathrm{ppm})$ and DMSO $(2.50 / 39.52 \mathrm{ppm})$ as internal standards. The description of signals include: $\mathrm{s}=$ singlet, $\mathrm{d}=$ doublet, $\mathrm{t}=$ triplet, $\mathrm{q}=$ quartet, $\mathrm{m}=$ multiplet and $\mathrm{br}$. = broad. All coupling constants are absolute values and $J$ values are expressed in Hertz (Hz). Mass spectra (MS and HRMS) were determined in the chemistry department of the University Heidelberg under the direction of Dr. J. Gross. FAB (+) spectra were obtained using a JEOL JMS-700 spectrometer. For the FAB-matrix, 3nitrobenzyl alcohol (NBA) or o-nitrophenyl octyl ether (NPOE) was used. For ESI (+) spectra, a ApexQe FT-ICR-MS spectrometer was used. Infrared Spectroscopy (IR) was processed on an FT-IR (IF528), IR (283) or FT-IR Vektor 22. Melting points were measured in open glass capillaries in a Büchi melting point apparatus (according to Dr. Tottoli) and were not corrected. Bis(trifluoromethyl) benziodoxole ${ }^{1}$ and Au complex $7^{2}$ were prepared according to the reported procedures. 


\section{Synthesis and characterization of (Phen)LAu ${ }^{\mathrm{I} N T f_{2}}$ complexes}

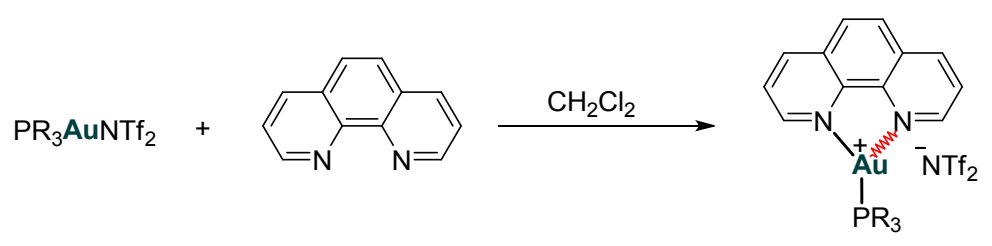

Phen $(3.6 \mathrm{mg}, 0.02 \mathrm{mmol})$ and $\mathrm{R}_{3} \mathrm{PAu}^{\mathrm{I}} \mathrm{NTf}_{2}(0.02 \mathrm{mmol})$ were dissolved in DCM $(1 \mathrm{~mL})$. The solvent was removed under vacuum and the residue was purified by crystallization with $\mathrm{DCM}$ and $\mathrm{Et}_{2} \mathrm{O}$ to afford the product of $(\mathrm{Phen}) \mathrm{PR}_{3} \mathrm{Au}^{\mathrm{I}} \mathrm{NTf}_{2}$ quantitatively.

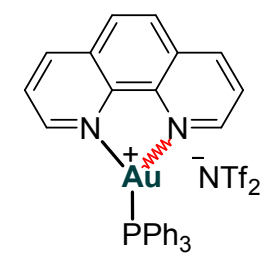

1a

M.p. $235-236{ }^{\circ} \mathrm{C} .{ }^{1} \mathbf{H}$ NMR $\left(400 \mathrm{MHz}, \mathrm{CD}_{2} \mathrm{Cl}_{2}\right) \delta 9.00(\mathrm{dd}, J=4.7,1.5 \mathrm{~Hz}, 2 \mathrm{H}), 8.66(\mathrm{dd}, J=8.2,1.6 \mathrm{~Hz}, 2 \mathrm{H}), 8.11(\mathrm{~s}, 2 \mathrm{H}), 8.03$ (dd, $J=8.2,4.7 \mathrm{~Hz}, 2 \mathrm{H}), 7.75-7.54(\mathrm{~m}, 15 \mathrm{H}) .{ }^{13} \mathbf{C}$ NMR $\left(101 \mathrm{MHz}, \mathrm{CD}_{2} \mathrm{Cl}_{2}\right) \delta 151.88$ (d, 2C), 143.28 (s, 2C), 140.14 (d, 2C), $134.43\left(\mathrm{~d}, \mathrm{~d}: J_{C-P}=14.0 \mathrm{~Hz}, 6 \mathrm{C}\right), 132.71\left(\mathrm{~d}, \mathrm{~d}: J_{C-P}=2.6 \mathrm{~Hz}, 3 \mathrm{C}\right), 130.75$ (s, 2C), 129.92 (d, d: $\left.J_{C-P}=11.9 \mathrm{~Hz}, 6 \mathrm{C}\right), 129.66$ (s, d: $\left.J_{C-P}=64.0 \mathrm{~Hz}, 3 \mathrm{C}\right), 128.17(\mathrm{~d}, 2 \mathrm{C}), 125.84(\mathrm{~d}, 2 \mathrm{C}), 120.40\left(\mathrm{CF}_{3}\right.$, q: $\left.J_{C-F}=321.4 \mathrm{~Hz}, 2 \mathrm{C}\right) .{ }^{31} \mathbf{P} \mathbf{N M R}(122 \mathrm{MHz}, \mathrm{CD} 2 \mathrm{Cl} 2) \delta 31.17$ (s). IR (ATR): $v\left(\mathrm{~cm}^{-1}\right)=3061,1776,1621,1593,1513,1481,1435,1352,1334,1177,1135,1102,1047,999,864,844$, 793, 748, 724, 711, 694, 612. HRMS [ESI, $\left(\mathrm{M}-\mathrm{NTf}_{2}\right)^{+}$]: m/z calcd. for $\mathrm{C}_{30} \mathrm{H}_{23} \mathrm{AuN}_{2} \mathrm{P}$ 639.1267, found 639.1264.

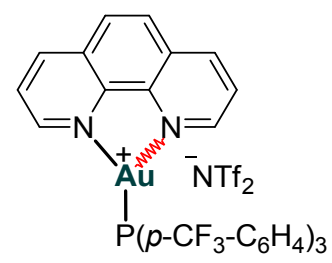

1b

M.p. $187-188{ }^{\circ} \mathrm{C} .{ }^{1} \mathbf{H}$ NMR $\left(600 \mathrm{MHz}, \mathrm{CD}_{2} \mathrm{Cl}_{2}\right) \delta 8.94(\mathrm{dd}, J=4.7,1.4 \mathrm{~Hz}, 2 \mathrm{H}), 8.62(\mathrm{dd}, J=8.2,1.4 \mathrm{~Hz}, 2 \mathrm{H}), 8.06(\mathrm{~s}, 2 \mathrm{H}), 8.01$ (dd, $J=8.1,4.7 \mathrm{~Hz}, 2 \mathrm{H}), 7.85-7.79(\mathrm{~m}, 6 \mathrm{H}), 7.75(\mathrm{dd}, J=13.0,8.3 \mathrm{~Hz}, 6 \mathrm{H}) .{ }^{13} \mathbf{C} \mathbf{N M R}\left(151 \mathrm{MHz}, \mathrm{CD}_{2} \mathrm{Cl}_{2}\right) \delta 152.00(\mathrm{~d}, 2 \mathrm{C})$, 143.03 (s, 2C), 140.48 (d, 2C), 134.92 (d, d: $\left.J_{C-P}=15.0 \mathrm{~Hz}, 6 \mathrm{C}\right), 134.80$ (s, qd: $J_{C-F, C-P}=33.3,2.6 \mathrm{~Hz}, 3 \mathrm{C}$ ), 132.99 (s, d: $J_{C-P}=60.8$ $\mathrm{Hz}, 3 \mathrm{C}), 130.74$ (s, 2C), 128.21 (d, 2C), $127.19-126.95$ (s, dq: $\left.J_{C-P, C-F}=12.4,3.8 \mathrm{~Hz}, 3 \mathrm{C}\right), 126.11$ (d, 2C), $123.74\left(\mathrm{CF}_{3}\right.$, q: $J_{C-F}=$ $272.3 \mathrm{~Hz}), 120.28\left(\mathrm{CF}_{3}, \mathrm{q}: J_{C-F}=321.5 \mathrm{~Hz}\right) .{ }^{31} \mathbf{P}$ NMR $\left(122 \mathrm{MHz}, \mathrm{CD}_{2} \mathrm{Cl}_{2}\right) \delta 31.26$ (s). IR (ATR): $v\left(\mathrm{~cm}^{-1}\right)=1611,1516,1429$, 1401, 1323, 1230, 1189, 1125, 1059, 1014, 836, 788, 775, 726, 707, 638, 613. HRMS [ESI, $\left.\left(\mathrm{M}-\mathrm{NTf}_{2}\right)^{+}\right]$: m/z calcd. for $\mathrm{C}_{33} \mathrm{H}_{20} \mathrm{AuF}_{9} \mathrm{~N}_{2} \mathrm{P}$ 843.0881, found 843.0899.

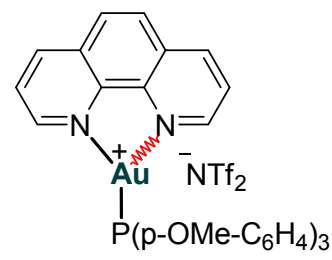

1c

M.p. $134-135^{\circ} \mathrm{C} .{ }^{1} \mathrm{H}$ NMR $\left(600 \mathrm{MHz}, \mathrm{CD}_{2} \mathrm{Cl}_{2}\right) \delta 8.93(\mathrm{dd}, J=4.6,1.3 \mathrm{~Hz}, 2 \mathrm{H}), 8.56(\mathrm{~d}, J=8.1 \mathrm{~Hz}, 2 \mathrm{H}), 8.01(\mathrm{~s}, 2 \mathrm{H}), 7.93(\mathrm{dd}, J$ $=8.1,4.7 \mathrm{~Hz}, 2 \mathrm{H}), 7.50(\mathrm{dd}, J=12.9,8.8 \mathrm{~Hz}, 6 \mathrm{H}), 6.99(\mathrm{dd}, J=8.7,1.7 \mathrm{~Hz}, 6 \mathrm{H}), 3.79(\mathrm{~s}, 9 \mathrm{H}) .{ }^{13} \mathbf{C} \mathbf{~ N M R}\left(151 \mathrm{MHz}, \mathrm{CD}_{2} \mathrm{Cl}_{2}\right) \delta$ 
163.07 (s, d: $J_{C-P}=2.4 \mathrm{~Hz}, 3 \mathrm{C}$ ), 151.78 (d, 2C), 143.38 (s, 2C), 139.87 (d, 2C), 135.80 (d, d: $\left.J_{C-P}=15.6 \mathrm{~Hz}, 6 \mathrm{C}\right), 130.63$ (s, 2C), $128.06(\mathrm{~d}, 2 \mathrm{C}), 125.66$ (d, 2C), $121.05\left(\mathrm{~s}, \mathrm{~d}: J_{C-P}=69.3 \mathrm{~Hz}, 3 \mathrm{C}\right), 120.34\left(\mathrm{CF}_{3}\right.$, q: $\left.J_{C-F}=321.5 \mathrm{~Hz}, 2 \mathrm{C}\right), 115.31$ (d, d: $J_{C-P}=13.1 \mathrm{~Hz}$, 6C), 55.99 (q, 3C). ${ }^{31} \mathbf{P}$ NMR (122 MHz, $\left.\mathrm{CD}_{2} \mathrm{Cl}_{2}\right) \delta 26.73$. IR (ATR): $v\left(\mathrm{~cm}^{-1}\right)=2844,1591,1570,1497,1460,1442,1427$, 1407, 1349, 1290, 1253, 1224, 1176, 1133, 1104, 1053, 1033, 1016, 863, 843, 833, 799, 771, 739, 724, 652, 639, 615. HRMS [ESI, $\left.(\mathrm{M}-\mathrm{NTf})_{2}\right)^{+}$]: m/z calcd. for $\mathrm{C}_{33} \mathrm{H}_{29} \mathrm{AuN}_{2} \mathrm{O}_{3} \mathrm{P} 729.1576$, found 729.1586 .

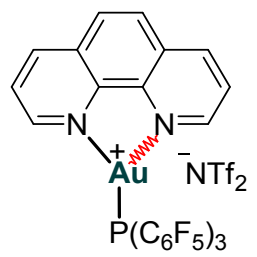

1d

${ }^{1}$ H NMR $(600 \mathrm{MHz}, \mathrm{DMSO}) \delta 9.18(\mathrm{~d}, \mathrm{~J}=4.6 \mathrm{~Hz}, 2 \mathrm{H}), 8.98(\mathrm{dd}, \mathrm{J}=8.2,1.2 \mathrm{~Hz}, 2 \mathrm{H}), 8.33(\mathrm{~s}, 2 \mathrm{H}), 8.28(\mathrm{dd}, \mathrm{J}=8.2,4.8 \mathrm{~Hz}, 2 \mathrm{H})$. The compound is not stable enough for ${ }^{13} \mathrm{C}$ NMR. IR (ATR): $v\left(\mathrm{~cm}^{-1}\right)=1647,1522,1479,1433,1391,1353,1336,1297$, $1181,1138,1097,1052,980,845,766,739,723,652,615$. Elemental anal. calcd. for $\mathrm{C}_{32} \mathrm{H}_{8} \mathrm{AuF}_{21} \mathrm{~N}_{3} \mathrm{O}_{4} \mathrm{PS}_{2} \cdot 1.5 \mathrm{H}_{2} \mathrm{O}: \mathrm{C} 31.59$, H 0.91, N 3.45, found: C 31.59, H 0.95, N 3.33.

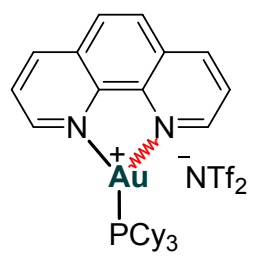

1e

M.p. $169-170{ }^{\circ} \mathrm{C} .{ }^{1} \mathbf{H}$ NMR $\left(600 \mathrm{MHz}, \mathrm{CD}_{2} \mathrm{Cl}_{2}\right) \delta 9.00(\mathrm{dd}, J=4.7,1.6 \mathrm{~Hz}, 2 \mathrm{H}), 8.56(\mathrm{dd}, J=8.1,1.4 \mathrm{~Hz}, 2 \mathrm{H}), 8.01(\mathrm{~s}, 2 \mathrm{H}), 7.97$ (dd, $J=8.1,4.7 \mathrm{~Hz}, 2 \mathrm{H}), 2.17-2.00(\mathrm{~m}, 9 \mathrm{H}), 1.89-1.81(\mathrm{~m}, 9 \mathrm{H}), 1.75-1.68(\mathrm{~m}, 3 \mathrm{H}), 1.57-1.47(\mathrm{~m}, 6 \mathrm{H}), 1.34(\mathrm{qt}, J=12.8,3.1$ $\mathrm{Hz}, 6 \mathrm{H}), 1.27-1.17$ (m, 3H). ${ }^{13} \mathrm{C}$ NMR (151 MHz, $\left.\mathrm{CD}_{2} \mathrm{Cl}_{2}\right) \delta 151.39$ (d, 2C), 143.35 (s, 2C), 139.78 (d, 2C), 130.66 (s, 2C), 128.06 $(\mathrm{d}, 2 \mathrm{C}), 125.65(\mathrm{~d}, 2 \mathrm{C}), 120.35\left(\mathrm{CF}_{3}, \mathrm{q}: J_{C-F}=321.5 \mathrm{~Hz}\right), 33.95\left(\mathrm{~d}, \mathrm{~d}: J_{C-P}=31.5 \mathrm{~Hz}, 3 \mathrm{C}\right), 31.56(\mathrm{t}, 3 \mathrm{C}), 27.36\left(\mathrm{t}, \mathrm{d}: J_{C-P}=12.3 \mathrm{~Hz}\right.$, 6C), 26.35 (t, d: $\left.J_{C-P}=1.1 \mathrm{~Hz}, 6 \mathrm{C}\right) .{ }^{31} \mathbf{P}$ NMR $(122 \mathrm{MHz}, \mathrm{CD} 3 \mathrm{CN}) \delta 54.51$ (s). IR (ATR): $v\left(\mathrm{~cm}^{-1}\right)=2931,2854,1624,1515$, 1445, 1427, 1356, 1333, 1264, 1226, 1175, 1137, 1102, 1054, 1004, 889, 846, 791, 772, 760, 740, 725, 663, 637, 615. HRMS [ESI, $\left.\left(\mathrm{M}-\mathrm{NTf}_{2}\right)^{+}\right]: \mathrm{m} / \mathrm{z}$ calcd. for $\mathrm{C}_{30} \mathrm{H}_{41} \mathrm{AuN}_{2} \mathrm{P}$ 657.2668, found 657.2678 .

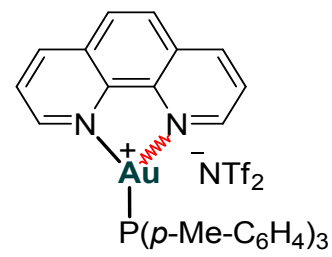

1f

M.p. $178-179{ }^{\circ} \mathrm{C} .{ }^{1} \mathbf{H}$ NMR $\left(600 \mathrm{MHz}, \mathrm{CD}_{2} \mathrm{Cl}_{2}\right) \delta 9.01(\mathrm{dd}, J=4.6,1.5 \mathrm{~Hz}, 2 \mathrm{H}), 8.61(\mathrm{dd}, J=8.1,1.0 \mathrm{~Hz}, 2 \mathrm{H}), 8.07(\mathrm{~s}, 1 \mathrm{H}), 7.98$ (dd, $J=8.1,4.6 \mathrm{~Hz}, 2 \mathrm{H}), 7.54(\mathrm{dd}, J=13.3,8.1 \mathrm{~Hz}, 6 \mathrm{H}), 7.37$ (dd, $J=8.1,2.1 \mathrm{~Hz}, 6 \mathrm{H}), 2.45(\mathrm{~s}, 9 \mathrm{H}) .{ }^{13} \mathbf{C} \mathbf{N M R}\left(151 \mathrm{MHz}, \mathrm{CD}_{2} \mathrm{Cl}_{2}\right)$ $\delta 151.64(\mathrm{~d}, 2 \mathrm{C}), 143.69$ (s, 2C), 143.41 (s, d: $\left.J_{C-P}=2.6 \mathrm{~Hz}, 3 \mathrm{C}\right), 139.55$ (d, 2C), 134.21 (d, d: $\left.J_{C-P}=14.2 \mathrm{~Hz}, 6 \mathrm{C}\right), 130.49$ (d, d: $\left.J_{C-P}=12.4 \mathrm{~Hz}, 6 \mathrm{C}\right), 130.48(\mathrm{~s}, 2 \mathrm{C}), 127.96(\mathrm{~d}, 2 \mathrm{C}), 126.57\left(\mathrm{~s}, \mathrm{~d}: J_{C-P}=65.5 \mathrm{~Hz}, 3 \mathrm{C}\right), 125.44(\mathrm{~d}, 2 \mathrm{C}), 120.34\left(\mathrm{CF}_{3}, \mathrm{q}: J_{C-F}=321.5\right.$ $\mathrm{Hz}, 2 \mathrm{C}), 21.63\left(\mathrm{t}, \mathrm{d}: J_{C-P}=1.2 \mathrm{~Hz}, 3 \mathrm{C}\right) .{ }^{31} \mathbf{P}$ NMR $\left(122 \mathrm{MHz}, \mathrm{CD}_{2} \mathrm{Cl}_{2}\right) \delta 28.91$ (s). IR (ATR): $v\left(\mathrm{~cm}^{-1}\right)=3070,2922,1599,1513$, 1499, 1427, 1400, 1348, 1225, 1177, 1135, 1102, 1054, 846, 807, 738, 725, 708, 651, 612. HRMS [ESI, (M-NTf $)^{+}{ }^{+}$: $\mathrm{m} / \mathrm{z}$ calcd. for $\mathrm{C}_{33} \mathrm{H}_{29} \mathrm{AuN}_{2} \mathrm{P} 681.1734$, found 681.1739 . 


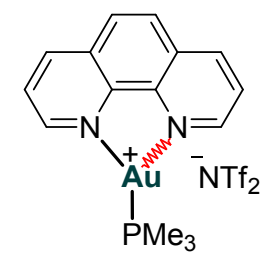

$1 \mathrm{~g}$

M.p. $212-213{ }^{\circ} \mathrm{C} .{ }^{1} \mathbf{H}$ NMR $\left(400 \mathrm{MHz}, \mathrm{CD}_{2} \mathrm{Cl}_{2}\right) \delta 9.12(\mathrm{dd}, J=4.7,1.6 \mathrm{~Hz}, 2 \mathrm{H}), 8.62(\mathrm{dd}, J=8.2,1.6 \mathrm{~Hz}, 2 \mathrm{H}), 8.07(\mathrm{~s}, 2 \mathrm{H}), 8.03$ $(\mathrm{dd}, J=8.2,4.7 \mathrm{~Hz}, 2 \mathrm{H}), 1.84(\mathrm{~d}, J=11.4 \mathrm{~Hz}, 9 \mathrm{H}) .{ }^{13} \mathbf{C} \mathbf{N M R}\left(101 \mathrm{MHz}, \mathrm{CD}_{2} \mathrm{Cl}_{2}\right) \delta 151.92(\mathrm{~d}, 2 \mathrm{C}), 143.31(\mathrm{~s}, 2 \mathrm{C}), 139.82(\mathrm{~d}, 2 \mathrm{C})$, $130.65(\mathrm{~s}, 2 \mathrm{C}), 128.03$ (d, 2C), $125.63(\mathrm{~d}, 2 \mathrm{C}), 120.41$ (q: $\left.J_{C-F}=321.5 \mathrm{~Hz}, 2 \mathrm{C}\right), 17.11$ (q, d: $\left.J_{C-P}=41.3 \mathrm{~Hz}, 3 \mathrm{C}\right) .{ }^{31} \mathbf{P}$ NMR (122 $\left.\mathrm{MHz}, \mathrm{CD}_{2} \mathrm{Cl}_{2}\right) \delta-15.60$. IR (ATR): $v\left(\mathrm{~cm}^{-1}\right)=3077,1802,1622,1574,1514,1427,1348,1328,1312,1228,1195,1178,1131$, 1056, 968, 862, 842, 788, 770, 741, 723, 692, 653, 618. HRMS [ESI, $\left(\mathrm{M}-\mathrm{NTf}_{2}\right)^{+}$]: m/z calcd. for $\mathrm{C}_{15} \mathrm{H}_{17} \mathrm{AuN}_{2} \mathrm{P} 453.0790$, found 453.0791 .

\section{Computed geometry of (Phen) $\mathrm{R}_{3} \mathrm{PAu}^{\mathrm{I}} \mathrm{NTf}_{2}$}

KS-DFT ( $\omega$ B97X-D3 functional ${ }^{3}$ with Ahlrichs def2-TZVP 4 basis set). Standard effective core potentials, ${ }^{5}$ CPCM solvent model with DCM and program of Orca 4.1.2 was used. ${ }^{6}$

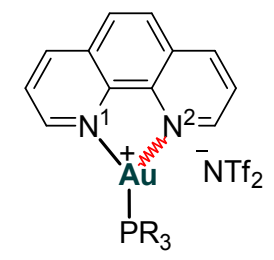

\begin{tabular}{lllll}
\hline $\mathrm{PR}_{3}$ & $\mathrm{Au}-\mathrm{N}^{1 /} \AA$ & $\mathrm{Au}-\mathrm{N}^{2} / \AA$ & $\mathrm{Au}-\mathrm{P} / \AA$ & $\theta /{ }^{\circ}$ \\
\hline $\mathrm{P}\left(p-\mathrm{OMe}-\mathrm{C}_{6} \mathrm{H}_{4}\right)_{3}$ & 2.16405 & 2.60588 & 2.26571 & 171.838 \\
$\mathrm{P}\left(p-\mathrm{Me}-\mathrm{C}_{6} \mathrm{H}_{4}\right)_{3}$ & 2.16064 & 2.60584 & 2.26491 & 172.782 \\
$\mathrm{PPh}_{3}$ & 2.16369 & 2.59973 & 2.26402 & 170.573 \\
$\mathrm{P}\left(p-\mathrm{F}-\mathrm{C}_{6} \mathrm{H}_{4}\right)_{3}$ & 2.16026 & 2.59658 & 2.26275 & 171.524 \\
$\mathrm{P}\left(p-\mathrm{Cl}_{-}-\mathrm{C}_{6} \mathrm{H}_{4}\right)_{3}$ & 2.15923 & 2.59114 & 2.26119 & 171.02 \\
$\mathrm{P}\left(p-\mathrm{CF}_{3}-\mathrm{C}_{6} \mathrm{H}_{4}\right)_{3}$ & 2.16186 & 2.57562 & 2.25819 & 169.118 \\
$\mathrm{P}\left(p-\mathrm{CN}-\mathrm{C}_{6} \mathrm{H}_{4}\right)_{3}$ & 2.15867 & 2.57256 & 2.25710 & 171.524 \\
$\mathrm{P}\left(\mathrm{C}_{6} \mathrm{~F}_{5}\right)_{3}$ & 2.27323 & 2.32891 & 2.24089 & 144.234 \\
$\mathrm{PCy}$ & 2.16837 & 2.64071 & 2.27695 & 170.18 \\
$\mathrm{PMe}$ & 2.17515 & 2.62113 & 2.26305 & 168.202 \\
$\mathrm{P}\left(o-\mathrm{Me}-\mathrm{C}_{6} \mathrm{H}_{4}\right)_{3}$ & 2.15348 & 2.63407 & 2.27694 & 174.387 \\
\hline
\end{tabular}


1) Computed geometry of (Phen)PPh $\mathrm{Au}^{+}$(1a)

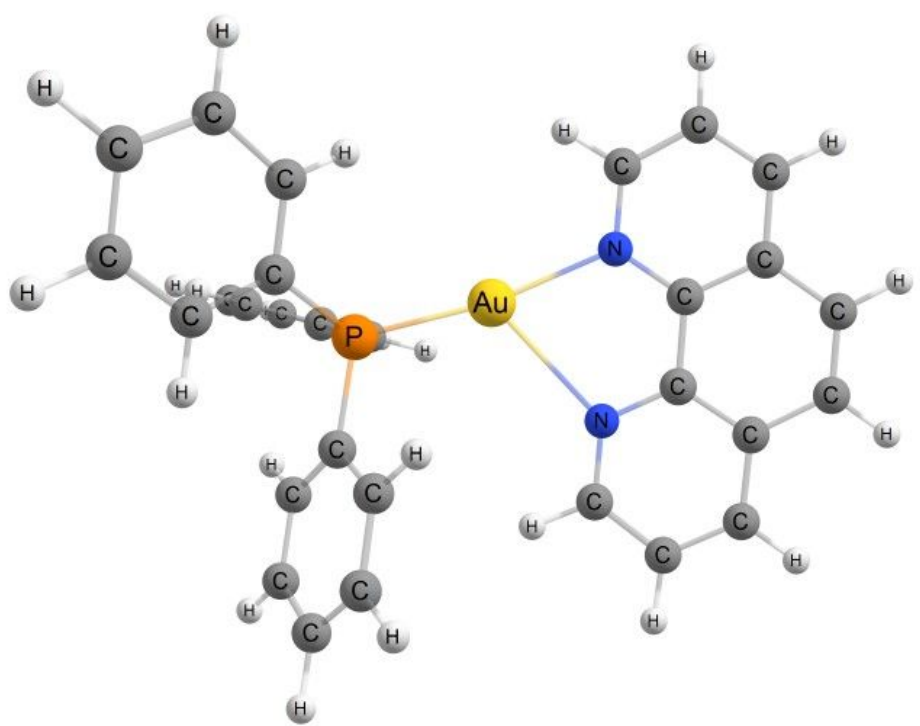

2) Computed geometry of (Phen) $\mathrm{P}\left(p-\mathrm{CF}_{3}-\mathrm{C}_{6} \mathrm{H}_{4}\right)_{3} \mathrm{Au}^{+}$(1b)

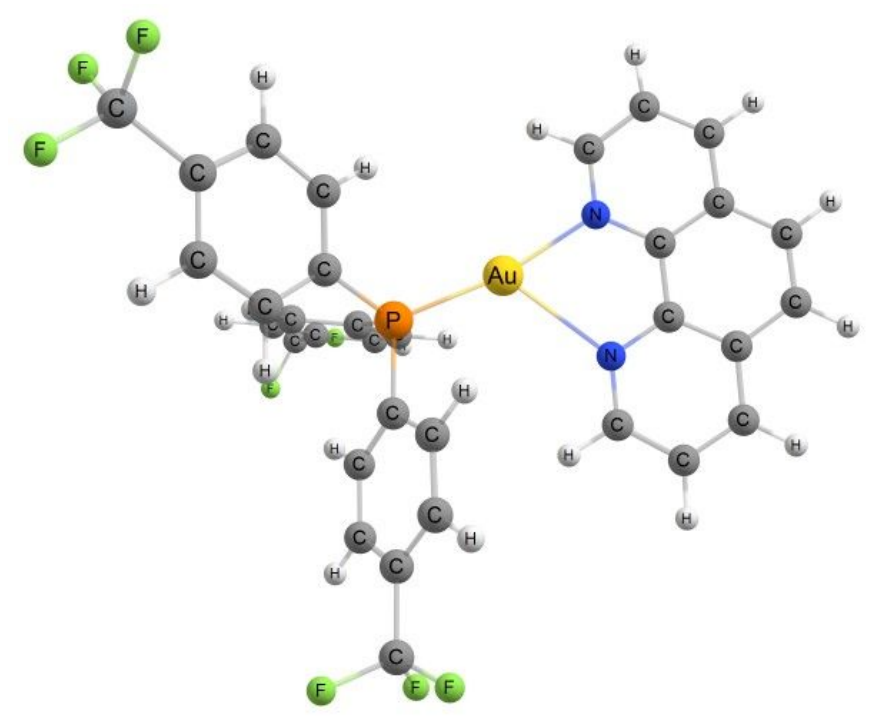

3) Computed geometry of (Phen) $\mathrm{P}\left(p-\mathrm{OMe}-\mathrm{C}_{6} \mathrm{H}_{4}\right)_{3} \mathrm{Au}^{+}$(1c) 


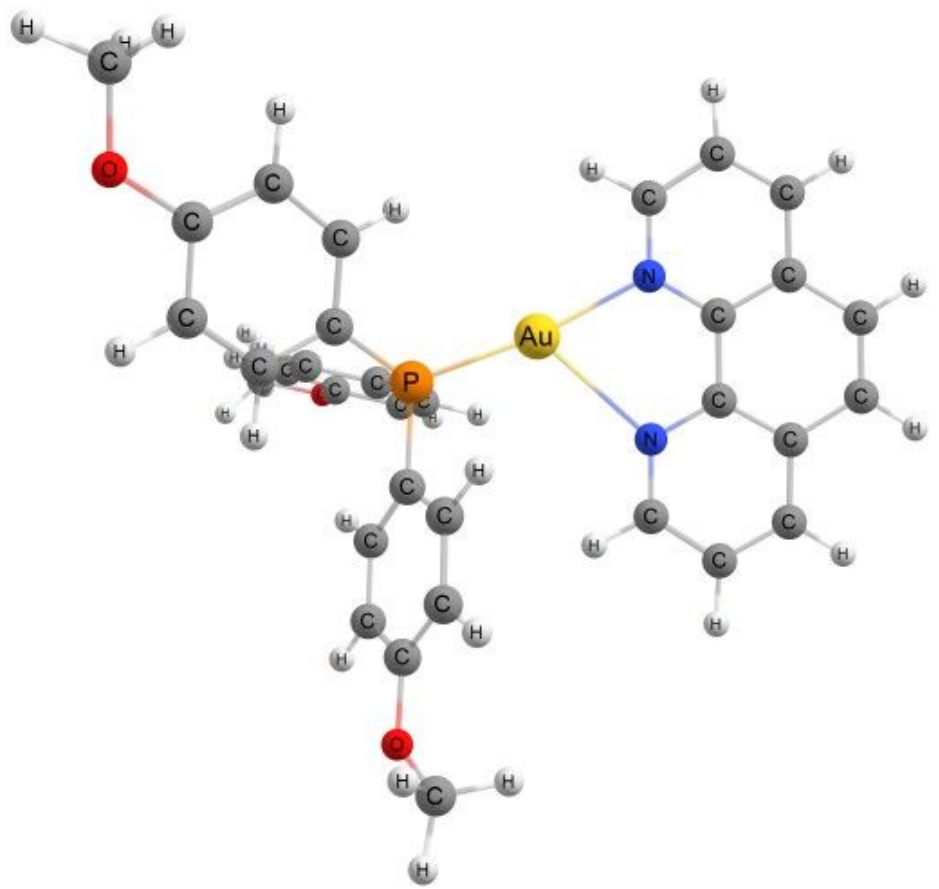

4) Computed geometry of (Phen) $\mathrm{P}\left(\mathrm{C}_{6} \mathrm{~F}_{5}\right)_{3} \mathrm{Au}^{+}$(1d)

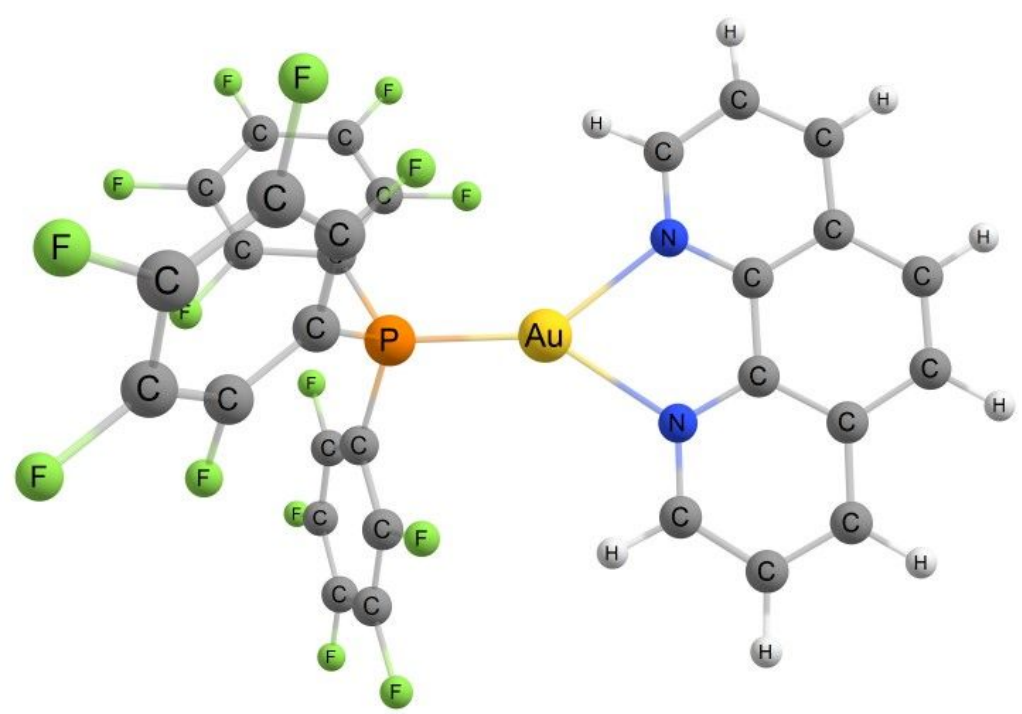

5) Computed geometry of (Phen) $\mathrm{PCy}_{3} \mathrm{Au}^{+}(\mathbf{1 e})$ 


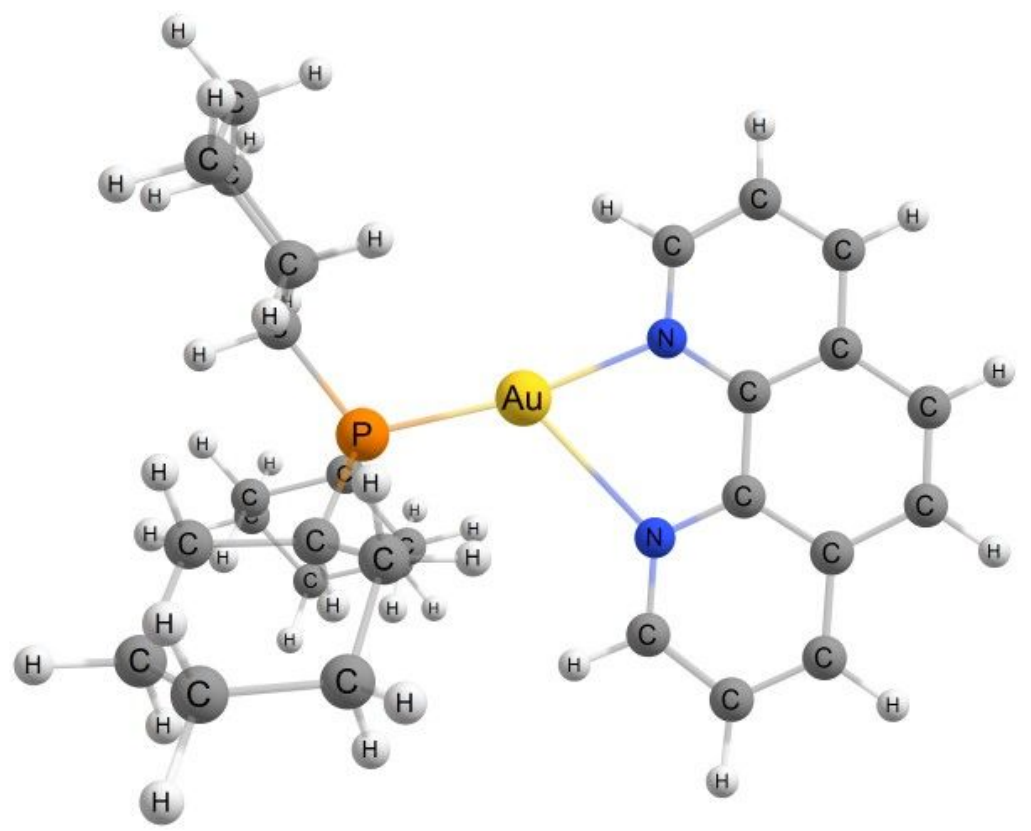

6) Computed geometry of (Phen) $\mathrm{P}\left(p-\mathrm{Me}-\mathrm{C}_{6} \mathrm{H}_{4}\right)_{3} \mathrm{Au}^{+}$(1f)

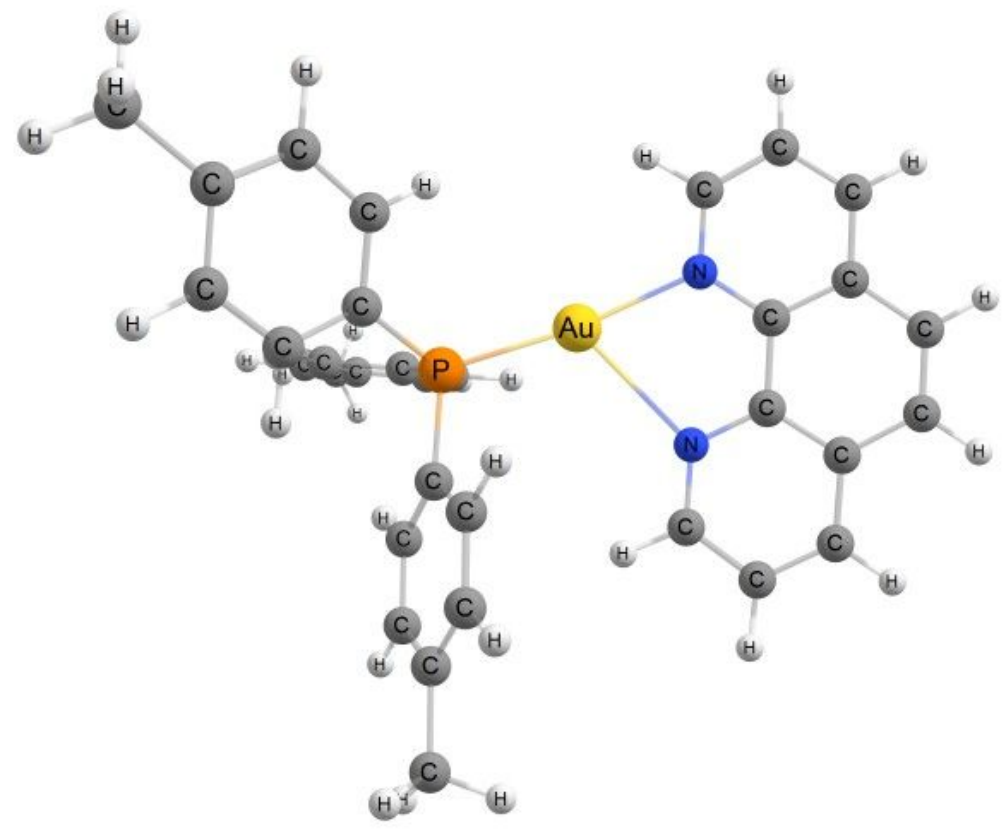

7) Computed geometry of (Phen) $\mathrm{PMe}_{3} \mathrm{Au}^{+}(\mathbf{1 g})$ 


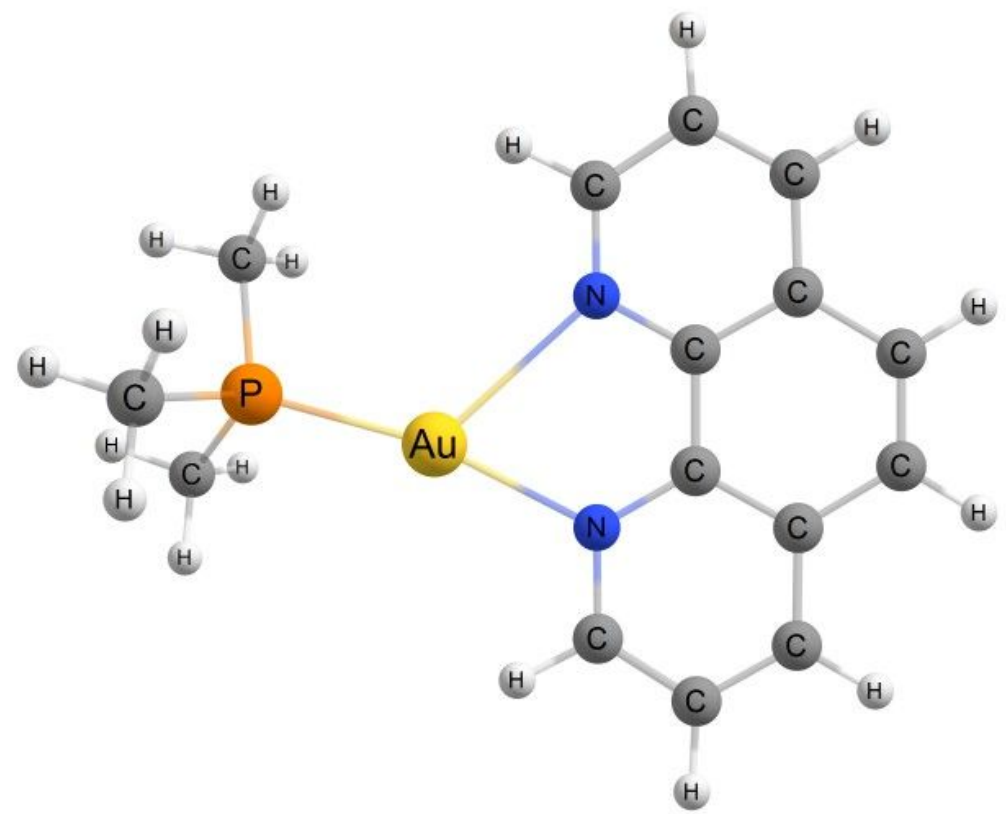

8) Computed geometry of (Phen) $\mathrm{P}\left(p-\mathrm{F}-\mathrm{C}_{6} \mathrm{H}_{4}\right)_{3} \mathrm{Au}^{+}$(1h)

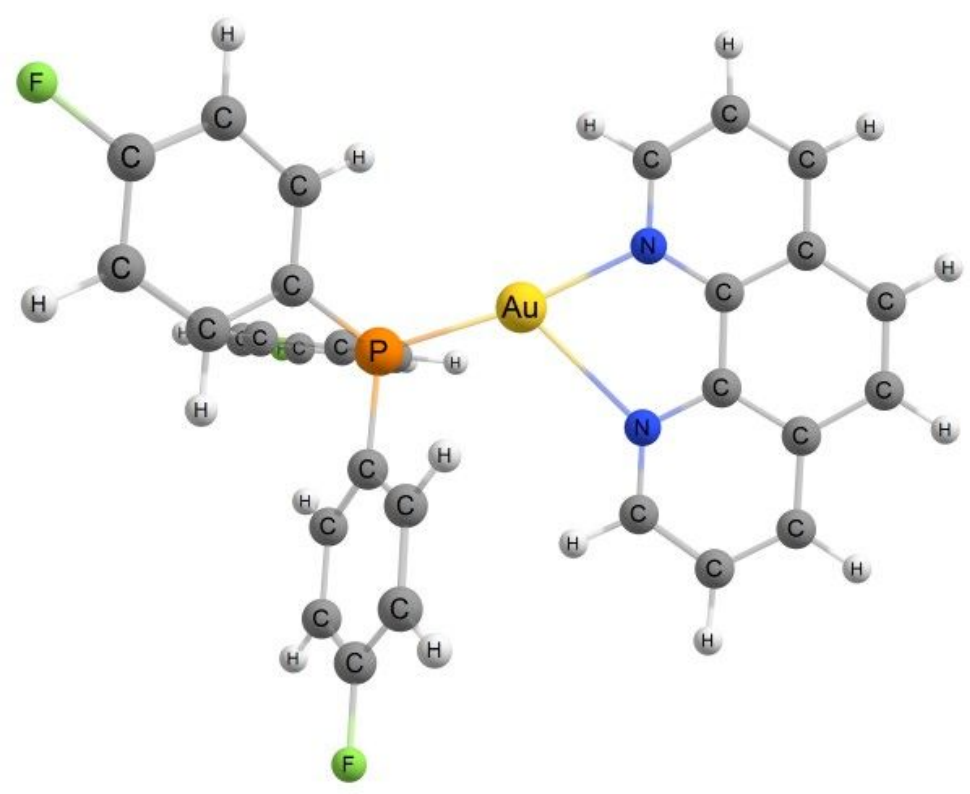

9) Computed geometry of (Phen) $\mathrm{P}\left(\mathrm{p}-\mathrm{Cl}-\mathrm{C}_{6} \mathrm{H}_{4}\right)_{3} \mathrm{Au}^{+}$(1i) 


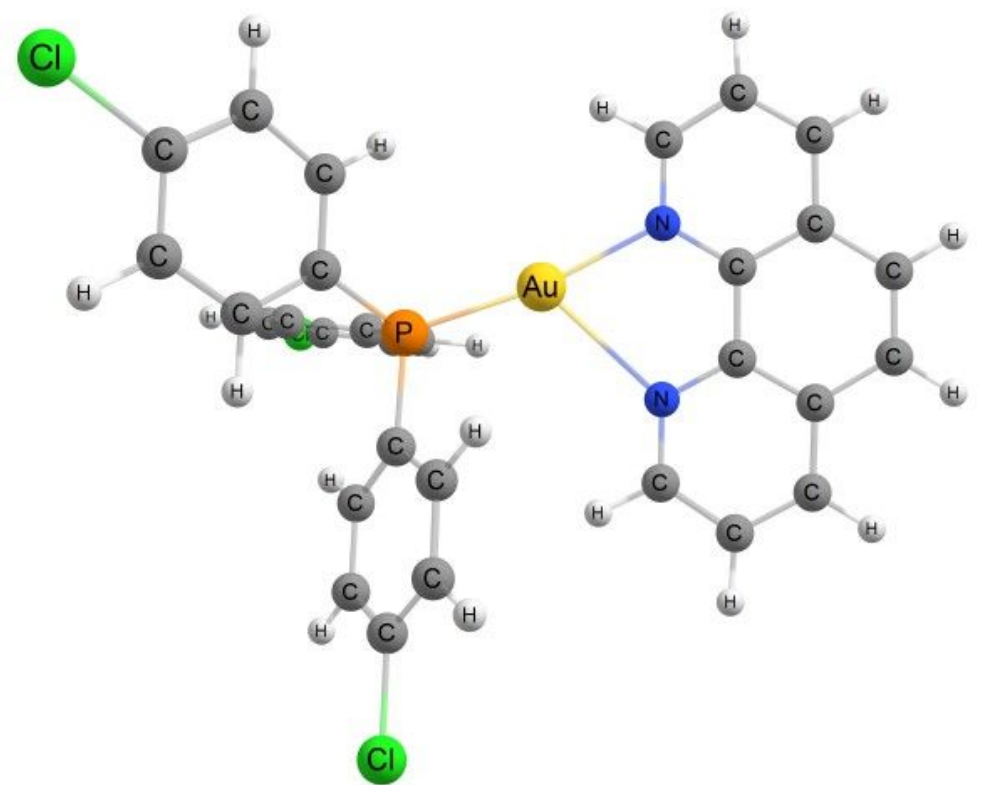

10) Computed geometry of (Phen) $\mathrm{P}\left(\mathrm{p}-\mathrm{CN}-\mathrm{C}_{6} \mathrm{H}_{4}\right)_{3} \mathrm{Au}^{+}(\mathbf{1 j})$

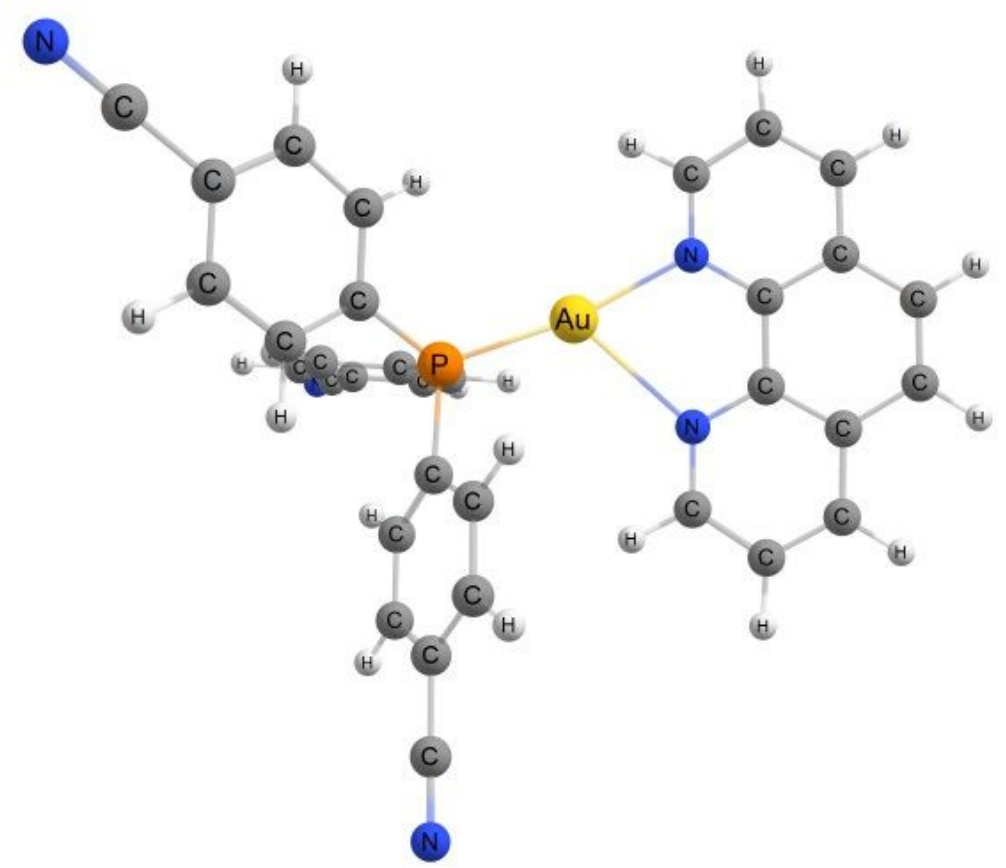

11) Computed geometry of (Phen) $\mathrm{P}\left(0-\mathrm{OMe}-\mathrm{C}_{6} \mathrm{H}_{4}\right)_{3} \mathrm{Au}^{+}(\mathbf{1} \mathbf{k})$ 


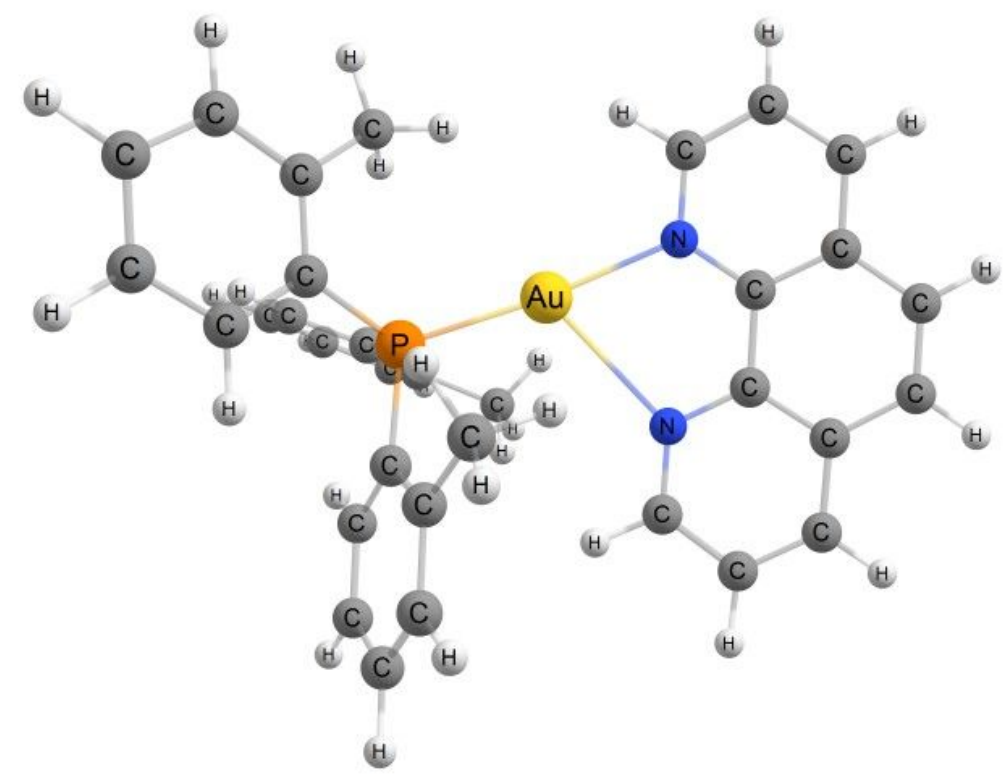

\section{Oxidative addition to asymmetric tri-coordinate gold(I) complexes}

4.1 Oxidative addition to (Phen) $\mathrm{Ph}_{3} \mathrm{PAu}{ }^{\mathrm{INTf}} \mathrm{N}_{2}$ in $\mathrm{CH}_{2} \mathrm{Cl}_{2}$

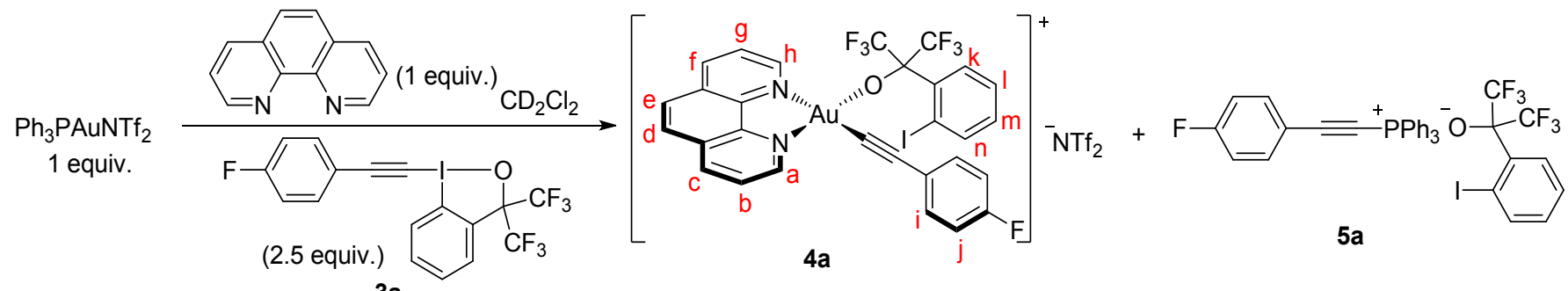

3a

In a glovebox, a J. Young NMR tube was charged with $\mathrm{Ph}_{3} \mathrm{PAuNTf}_{2}$ (14.8 mg, $0.02 \mathrm{mmol}$ ) and Phen (3.6 mg, $0.02 \mathrm{mmol}$ ). $\mathrm{CD}_{2} \mathrm{Cl}_{2}(0.5 \mathrm{~mL})$ was added to the tube, followed by addition of $\mathbf{3 a}(25 \mathrm{mg}, 0.05 \mathrm{mmol})$ and hexamethylbenzene (3.9 mg, $0.024 \mathrm{mmol}$ ) as an internal standard. The reaction was monitored by ${ }^{1} \mathrm{H},{ }^{19} \mathrm{~F}$, and ${ }^{31} \mathrm{P}$ NMR spectroscopy. HRMS (ESI, M ${ }^{+}$): $\mathrm{m} / \mathrm{z}$ calcd. for $\mathrm{C}_{29} \mathrm{H}_{16} \mathrm{AuF}_{7} \mathrm{IN}_{2} \mathrm{O} 846.9856$, found 846.9867.

${ }^{1} \mathbf{H}$ NMR $\left(\mathrm{CD}_{2} \mathrm{Cl}_{2}\right)$ spectrum of oxidative addition to (Phen) $\mathrm{Ph}_{3} \mathrm{PAu}^{\mathrm{INTf}}$ 


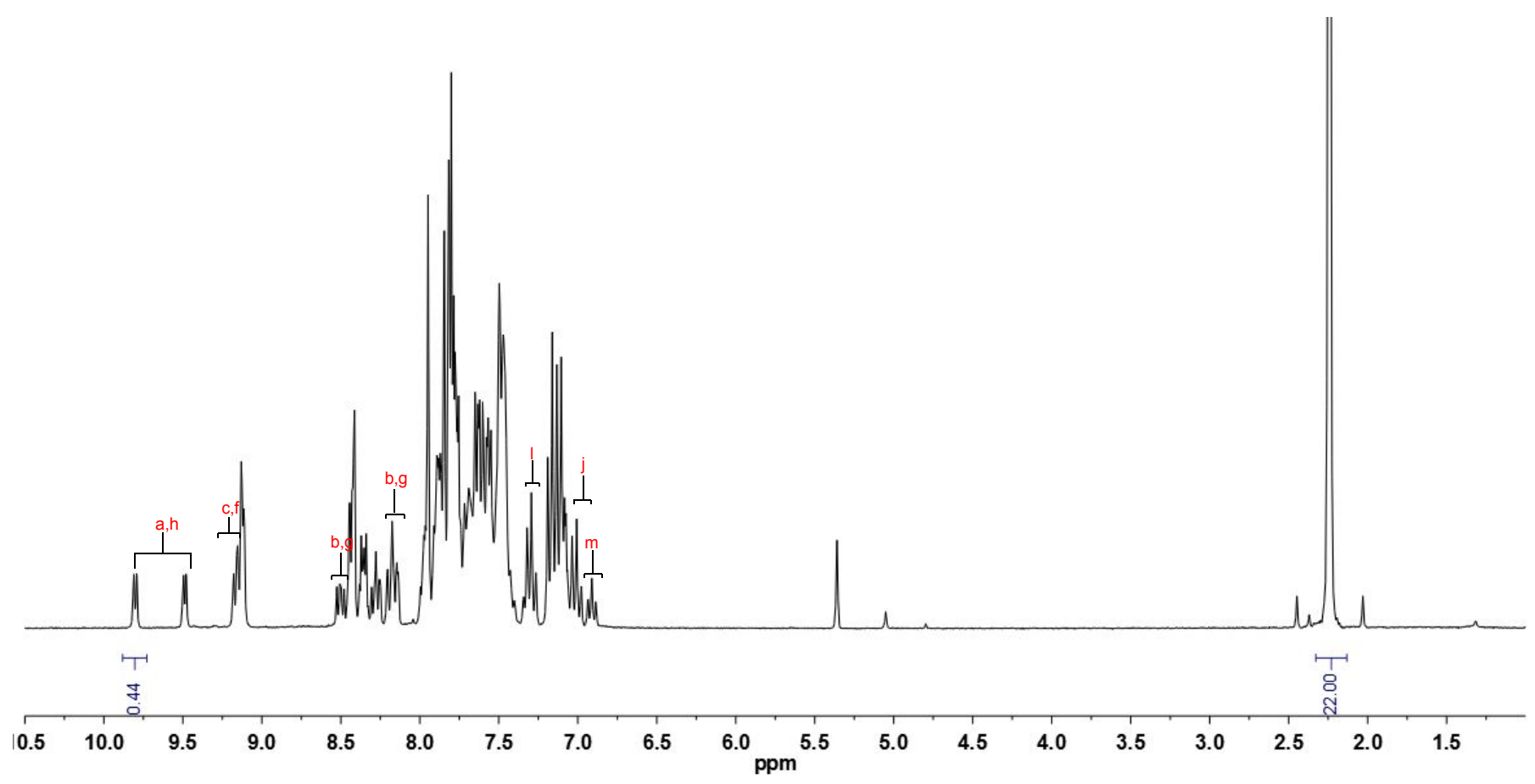

${ }^{31} \mathbf{P}$ NMR $\left(\mathrm{CD}_{2} \mathrm{Cl}_{2}\right)$ spectrum of oxidative addition to $(\mathrm{Phen}) \mathrm{Ph}_{3} \mathrm{PAu}^{\mathrm{I}} \mathrm{NTf}_{2}$

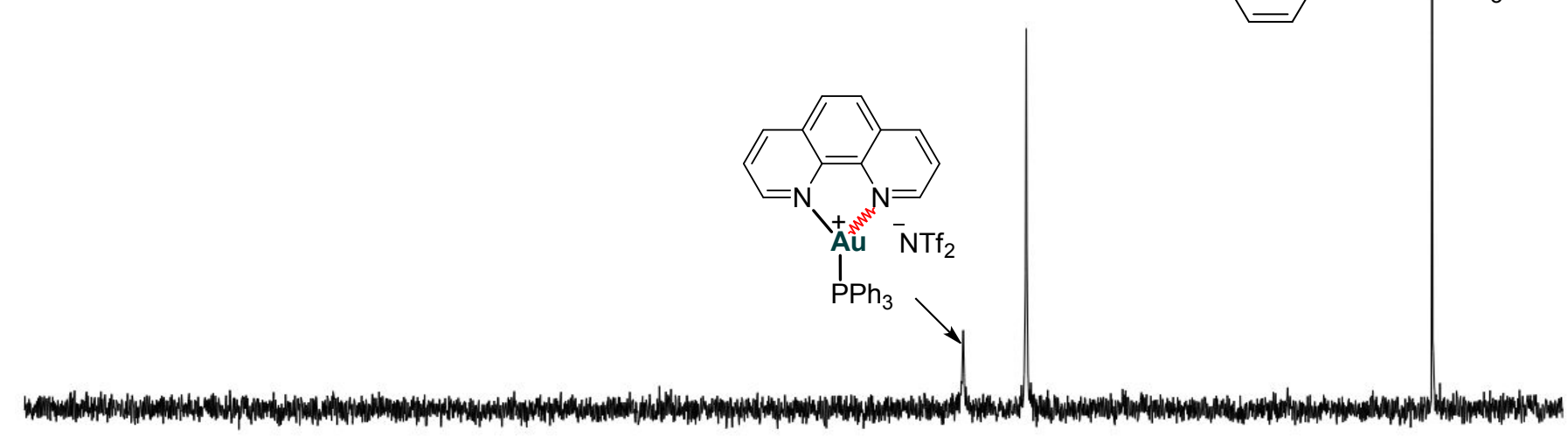

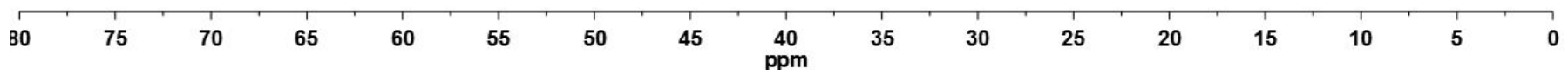


${ }^{19} \mathbf{F}$ NMR $\left(\mathrm{CD}_{2} \mathrm{Cl}_{2}\right)$ spectrum of oxidative addition to (Phen) $\mathrm{Ph}_{3} \mathrm{PAu}^{\mathrm{I}} \mathrm{NTf}_{2}$

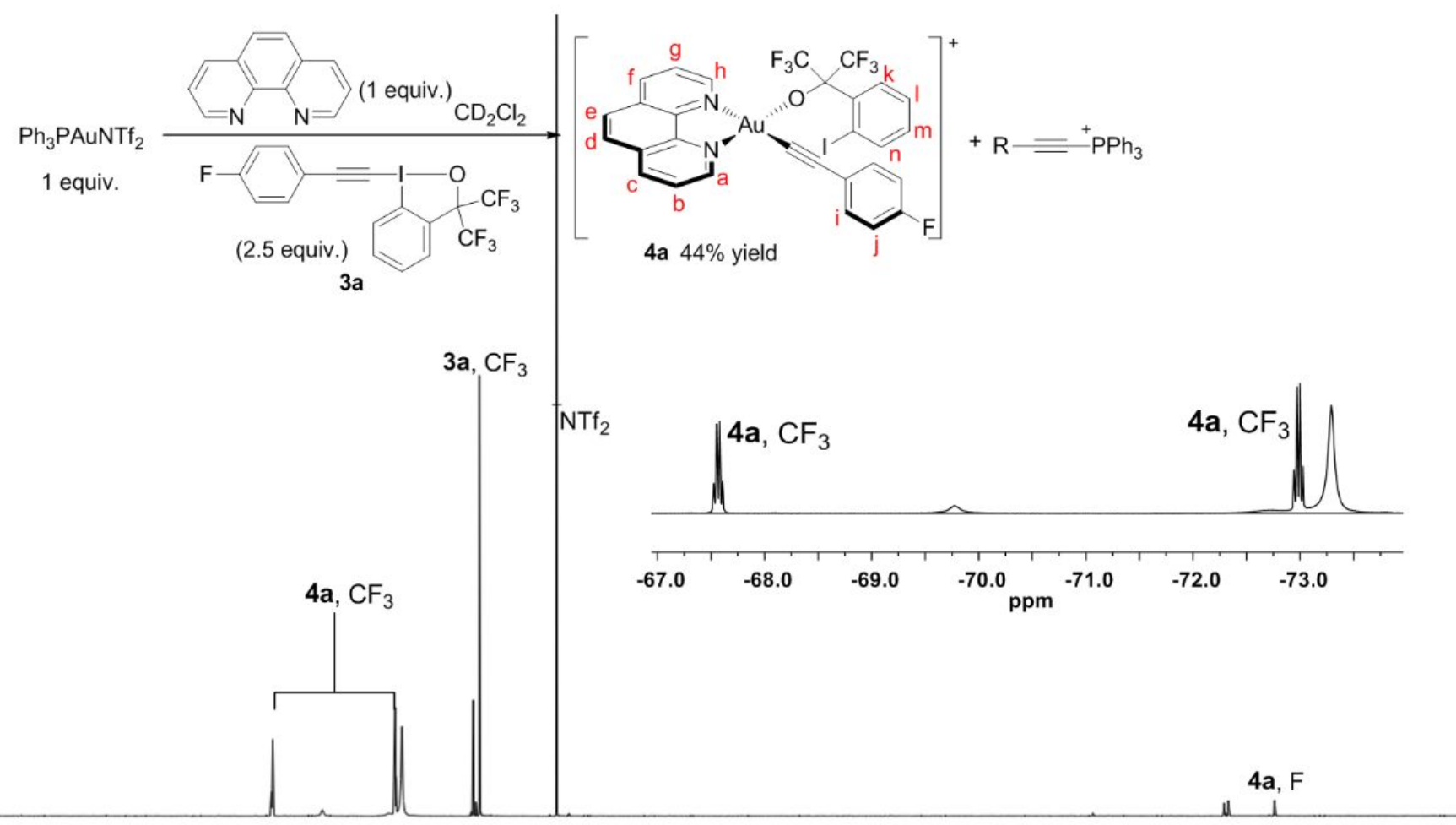

\begin{tabular}{|c|c|c|c|c|c|c|c|c|c|c|c|c|c|c|}
\hline-58 & -62 & -66 & -70 & -74 & -78 & -82 & -86 & -90 & -94 & -98 & -102 & -106 & -110 & -114 \\
\hline
\end{tabular}
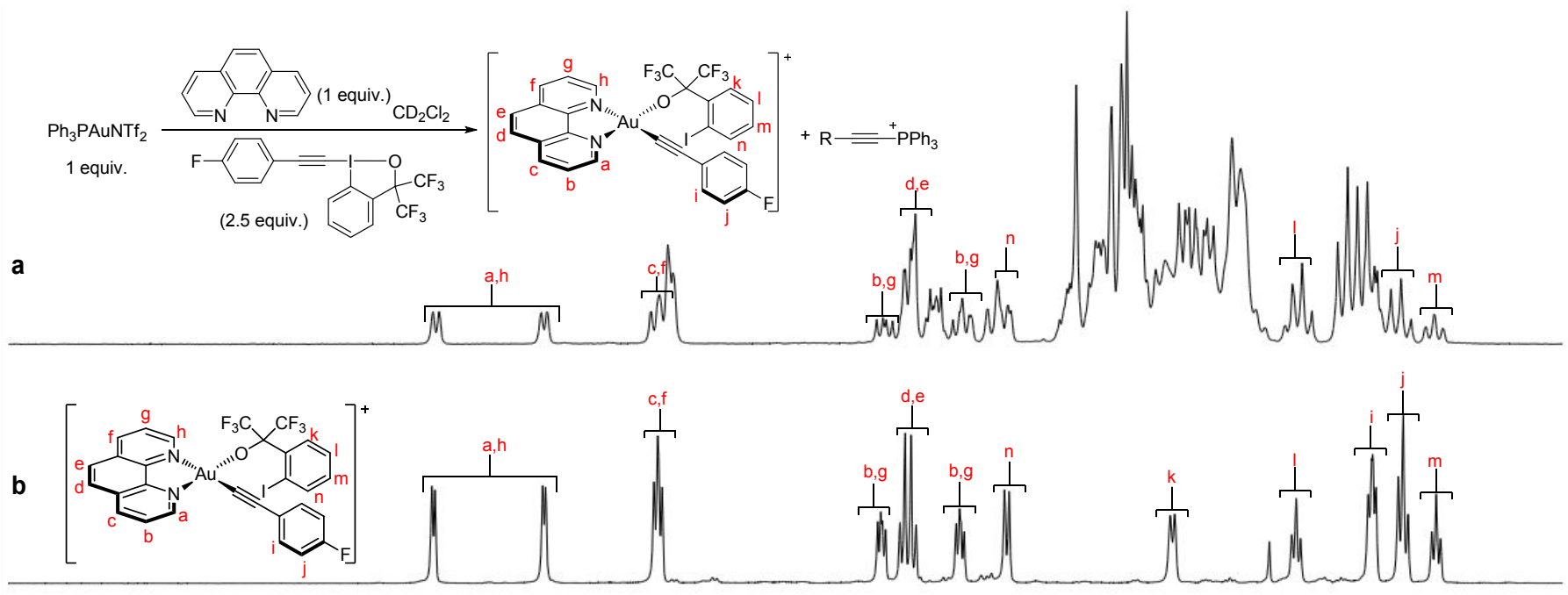

Figure S1. a) ${ }^{1} \mathrm{H}$ NMR spectrum of oxidative addition to (Phen) $\mathrm{Ph}_{3} \mathrm{PAu}^{\mathrm{I} N T f}$; b) ${ }^{1} \mathrm{H}$ NMR spectrum of gold(III) complex 4a. 


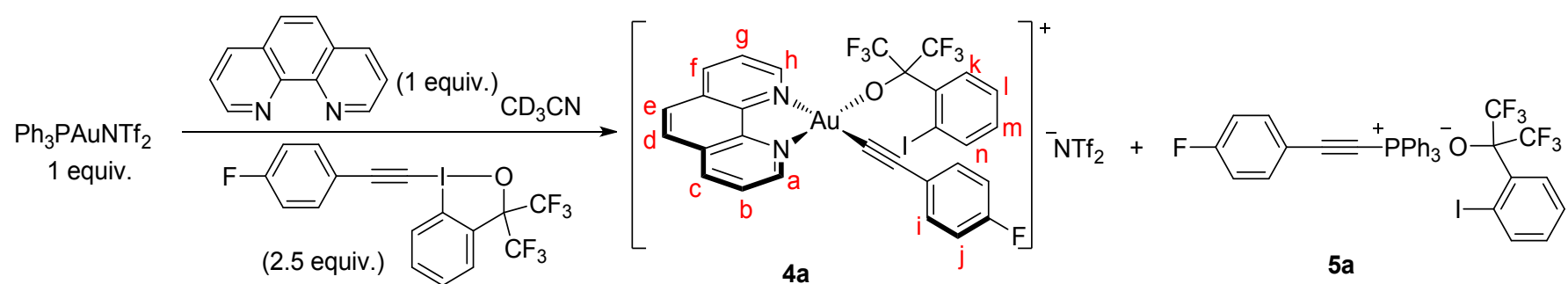

$3 a$

In a glovebox, a J. Young NMR tube was charged with $\mathrm{Ph}_{3} \mathrm{PAuNTf}_{2}(14.8 \mathrm{mg}, 0.02 \mathrm{mmol})$ and Phen $(3.6 \mathrm{mg}, 0.02 \mathrm{mmol})$. $\mathrm{CD}_{3} \mathrm{CN}(0.5 \mathrm{~mL})$ was added to the tube, followed by addition of $3 \mathrm{a}(25 \mathrm{mg}, 0.05 \mathrm{mmol})$ and $\mathrm{CH}_{2} \mathrm{Cl}_{2}(1.3 \mu \mathrm{L}, 0.02 \mathrm{mmol})$ as internal standard. The reaction was monitored by ${ }^{1} \mathrm{H}$ and ${ }^{31} \mathrm{P}$ NMR spectroscopy.

${ }^{1} \mathrm{H}$ NMR $\left(\mathrm{CD}_{3} \mathrm{CN}\right)$ spectrum of oxidative addition to (Phen) $\mathrm{Ph}_{3} \mathrm{PAu}^{\mathrm{I}} \mathrm{NTf}_{2}$ after 90 min reaction

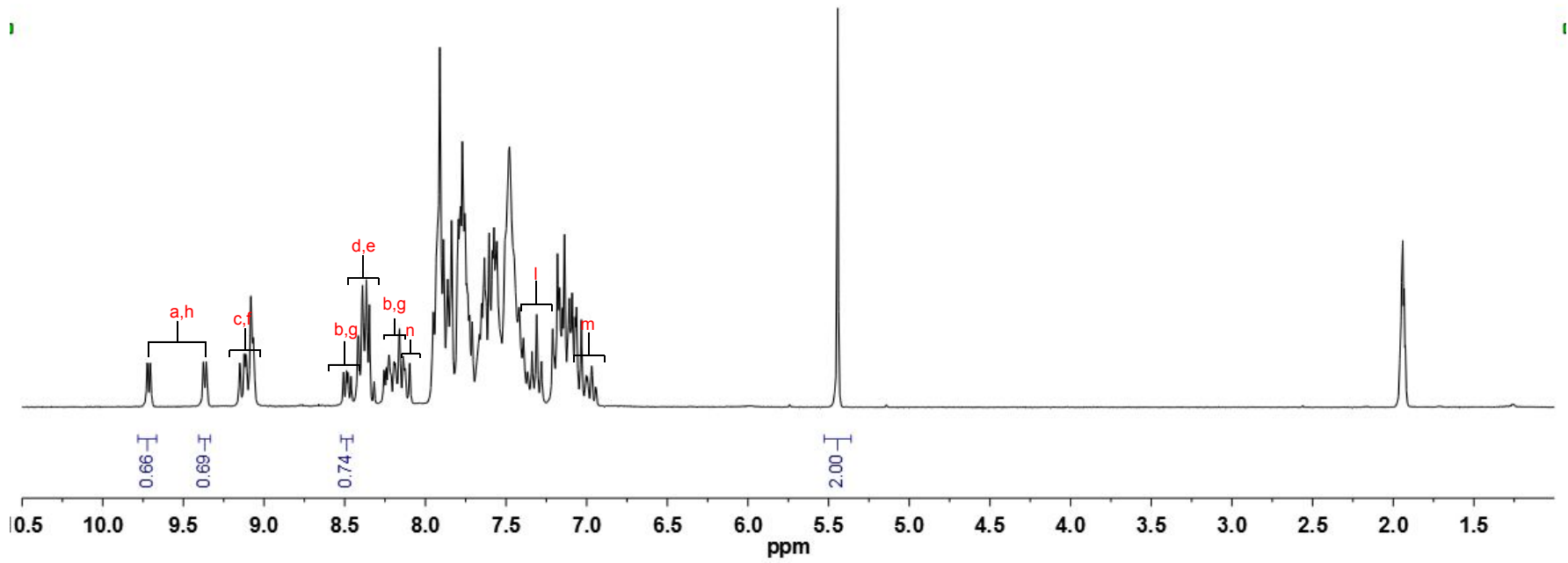

${ }^{31} \mathbf{P}$ NMR $\left(\mathrm{CD}_{3} \mathrm{CN}\right)$ spectrum of the oxidative addition to $(\mathrm{Phen}) \mathrm{Ph}_{3} \mathrm{PAu}^{\mathrm{I} N T f_{2}}$ after 90 min reaction

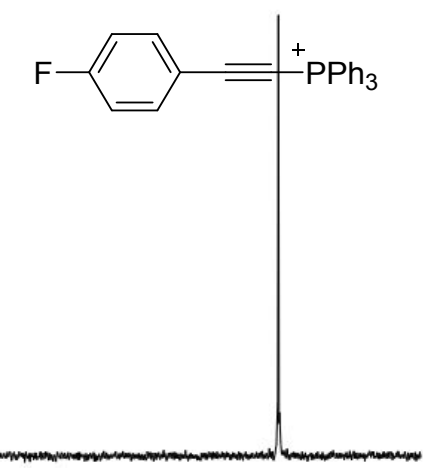

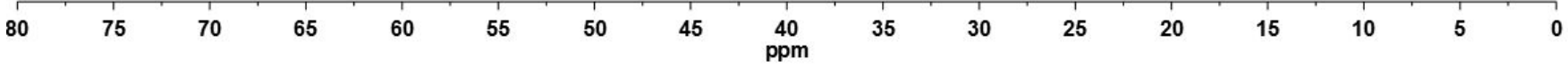


4.3 Oxidative addition to ( $\mathrm{Phen}) \mathrm{Ph}_{3} \mathrm{PAu}^{\mathrm{I} N T f_{2}}$ in $\mathrm{CH}_{3} \mathrm{CN}\left(0.6\right.$ eq. of $\left.\mathrm{H}_{2} \mathrm{O}\right)$

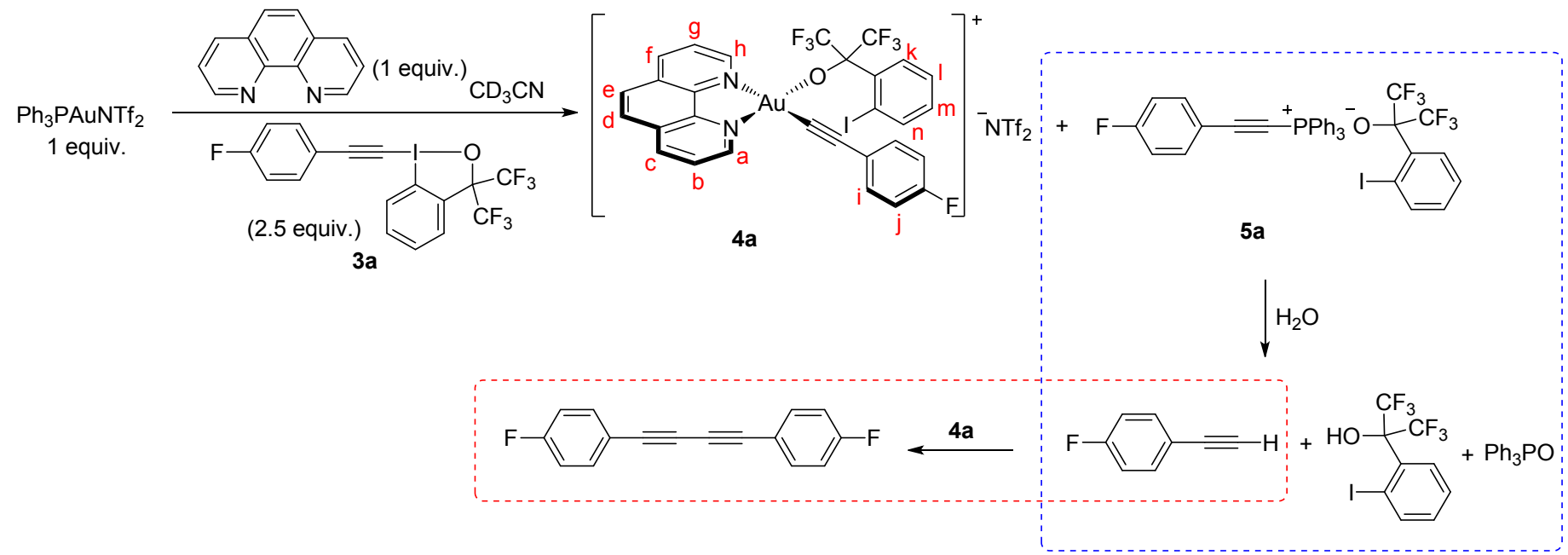

${ }^{1} \mathrm{H}$ NMR $\left(\mathrm{CD}_{3} \mathrm{CN}\right)$ spectrum of oxidative addition to ( $\left.\mathrm{Phen}\right) \mathrm{Ph}_{3} \mathrm{PAu}^{\mathrm{I}} \mathrm{NTf}_{2}\left(0.6\right.$ eq. of $\left.\mathrm{H}_{2} \mathrm{O}\right)$ after $1 \mathrm{~h}$ reaction

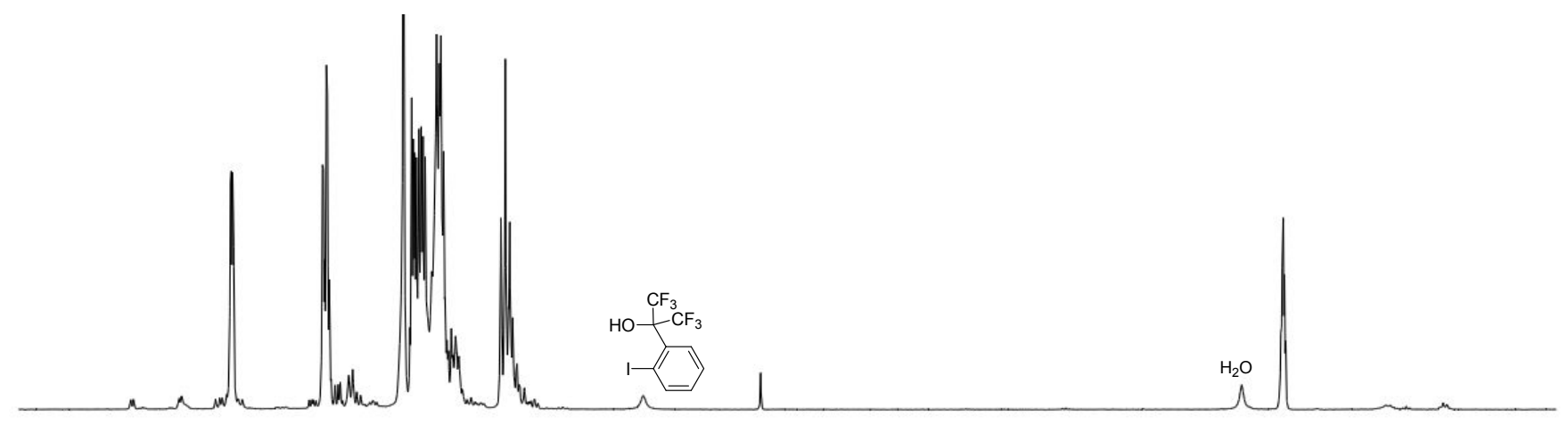

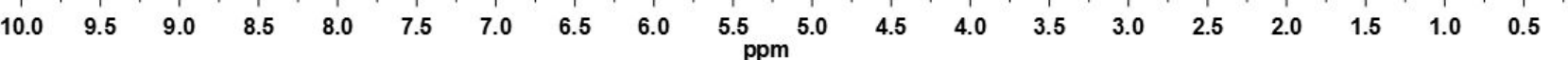

${ }^{31} \mathbf{P}$ NMR $\left(\mathrm{CD}_{3} \mathrm{CN}\right)$ spectrum of oxidative addition to ( $\left.\mathrm{Phen}\right) \mathrm{Ph}_{3} \mathrm{PAu}^{\mathrm{I}} \mathrm{NTf}_{2}\left(0.6\right.$ eq. of $\left.\mathrm{H}_{2} \mathrm{O}\right)$ after $1 \mathrm{~h}$ reaction

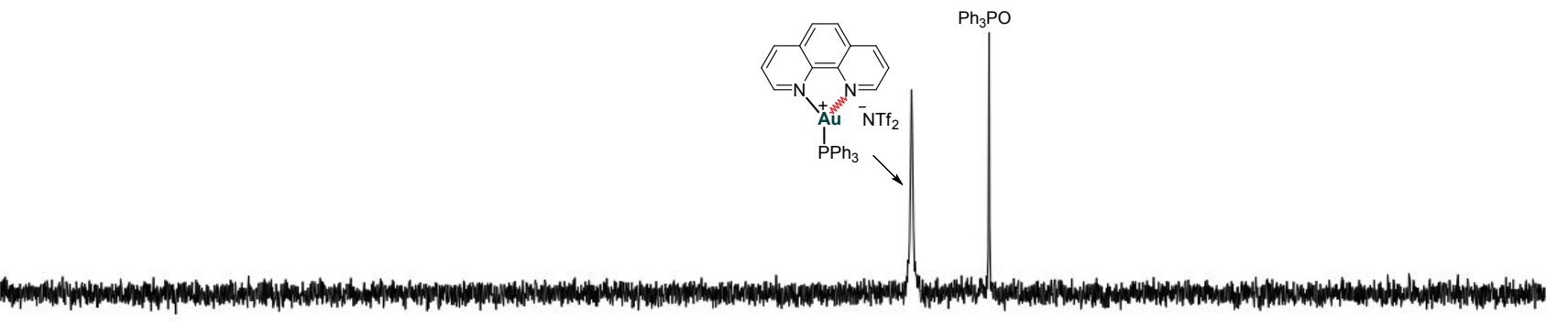

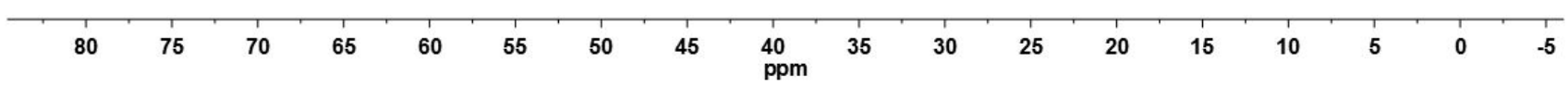


${ }^{1} \mathbf{H}$ NMR $\left(\mathrm{CD}_{3} \mathrm{CN}\right)$ spectrum of oxidative addition to (Phen) $\mathrm{Ph}_{3} \mathrm{PAu}^{\mathrm{I} N T f} \mathrm{~N}_{2}\left(0.6\right.$ eq. of $\left.\mathrm{H}_{2} \mathrm{O}\right)$ after 2 h reaction

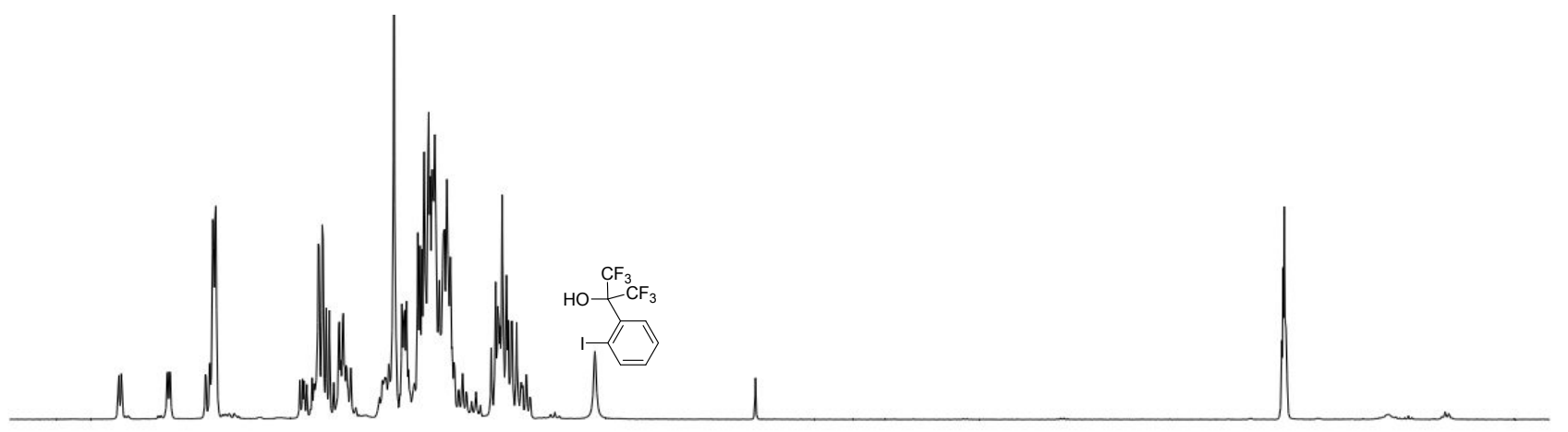

$\begin{array}{llllllllllllllllllll}10.0 & 9.5 & 9.0 & 8.5 & 8.0 & 7.5 & 7.0 & 6.5 & 6.0 & 5.5 & 5.0 & 4.5 & 4.0 & 3.5 & 3.0 & 2.5 & 2.0 & 1.5 & 1.0 & 0.5\end{array}$

${ }^{31} \mathrm{P}$ NMR $\left(\mathrm{CD}_{3} \mathrm{CN}\right)$ spectrum of oxidative addition to (Phen) $\mathrm{Ph}_{3} \mathrm{PAu}^{\mathrm{I} N T f} \mathrm{NT}_{2}\left(0.6\right.$ eq. of $\left.\mathrm{H}_{2} \mathrm{O}\right)$ after 2 h reaction

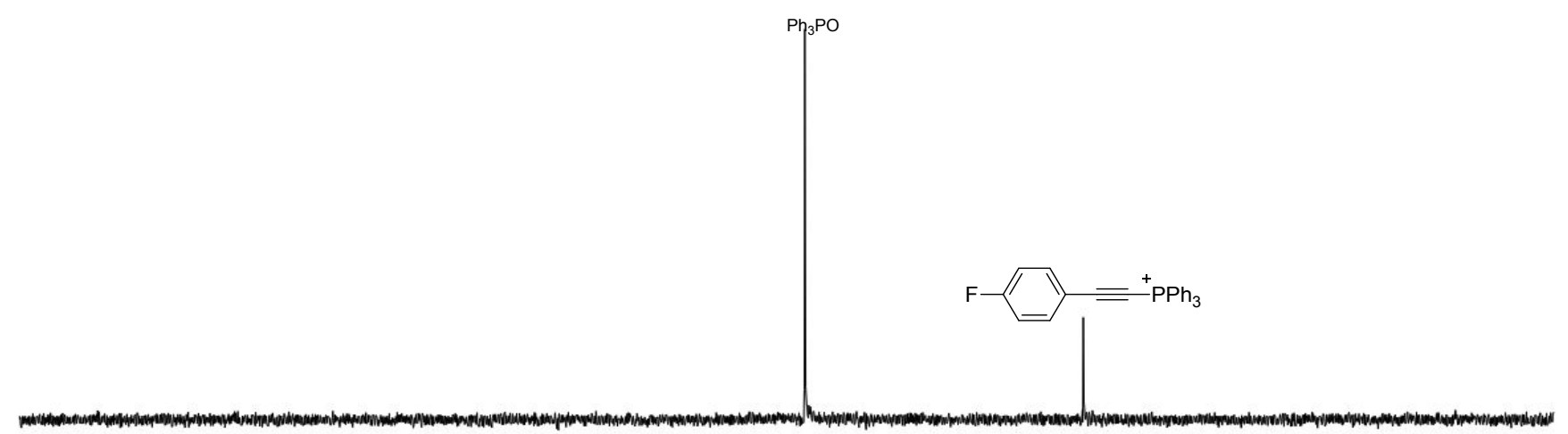

\subsection{Oxidative addition to (2,2'-bipyridyl) $\mathrm{Ph}_{3} \mathrm{PAu}^{\mathrm{I}} \mathrm{NTf}_{2}$}

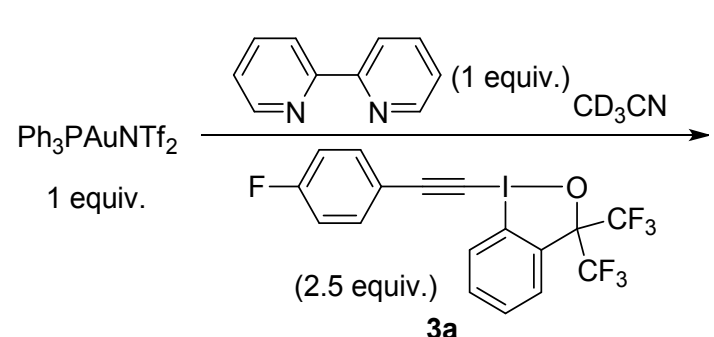

$3 a$

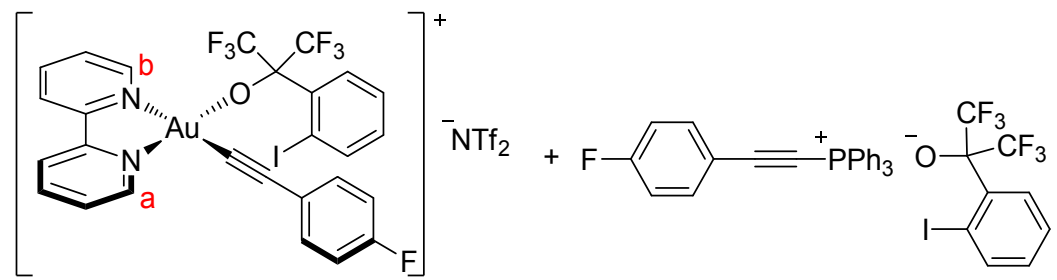

4b $35 \%$ yield

In a glovebox, a J. Young NMR tube was charged with $\mathrm{Ph}_{3} \mathrm{PAuNTf}_{2}$ (14.8 mg, $0.02 \mathrm{mmol}$ ) and bpy (3.12 mg, $0.02 \mathrm{mmol}$ ). $\mathrm{CD}_{3} \mathrm{CN}(0.5 \mathrm{~mL})$ was added to the tube, followed by addition of $\mathbf{3 a}(25 \mathrm{mg}, 0.05 \mathrm{mmol})$ and hexamethylbenzene (3.25 mg, $0.02 \mathrm{mmol}$ ) as internal standard. The reaction was monitored by ${ }^{1} \mathrm{H}$ and ${ }^{31} \mathrm{P}$ NMR spectroscopy. 
${ }^{1} \mathbf{H}$ NMR $\left(\mathrm{CD}_{3} \mathrm{CN}\right)$ spectrum of the oxidative addition to (2,2'-bipyridyl) $\mathrm{Ph}_{3} \mathrm{PAu}^{\mathrm{I}} \mathrm{NTf}_{2}$

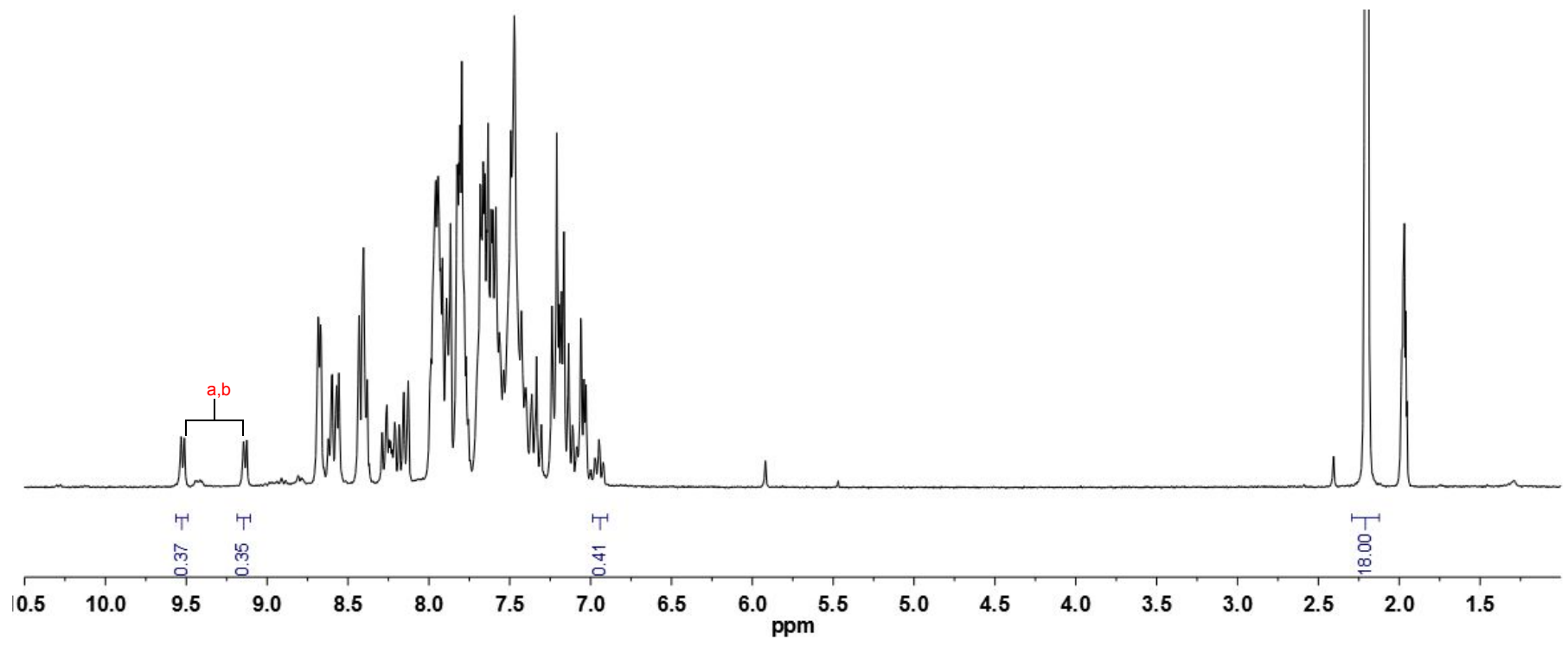

${ }^{31} \mathbf{P}$ NMR $\left(\mathrm{CD}_{3} \mathrm{CN}\right)$ spectrum of the oxidative addition to (2,2'-bipyridyl) $\mathrm{Ph}_{3} \mathrm{PAu}^{\mathrm{I}} \mathrm{NTf}_{2}$

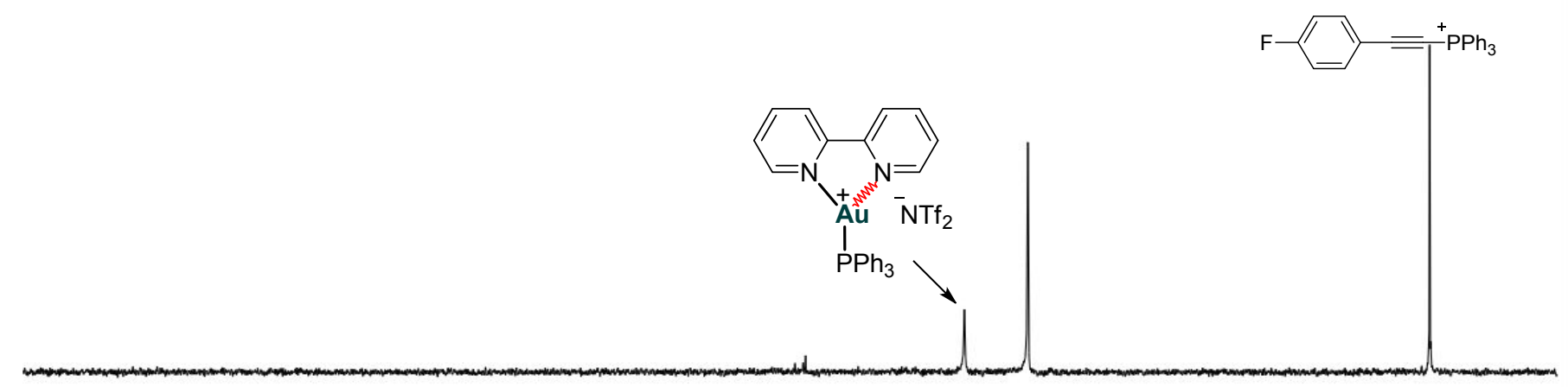

\begin{tabular}{|c|c|c|c|c|c|c|c|c|c|c|c|c|c|c|c|}
\hline 80 & 75 & 70 & 65 & 60 & 55 & 50 & 45 & $\begin{array}{c}1 \\
40 \\
\mathrm{ppm}\end{array}$ & 35 & 30 & 25 & 20 & 15 & 10 & 5 \\
\hline
\end{tabular}

\subsection{Oxidative addition to (Phen) $\left(\mathrm{C}_{6} \mathrm{~F}_{5}\right)_{3} \mathrm{PAu}^{\mathrm{I}} \mathrm{NTf}_{2}$ in $\mathrm{CH}_{2} \mathrm{Cl}_{2}$}

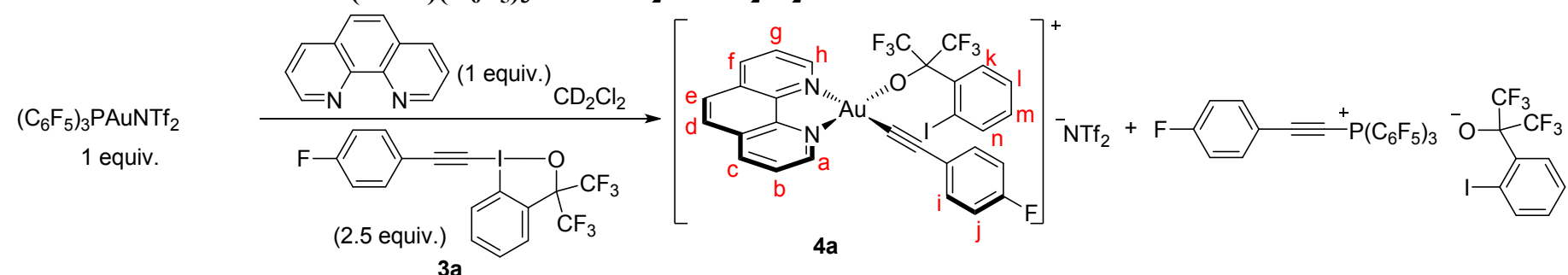

In a glovebox, a J. Young NMR tube was charged with $\left(\mathrm{C}_{6} \mathrm{~F}_{5}\right)_{3} \mathrm{PAuNTf}_{2}(5 \mathrm{mg}, 0.005 \mathrm{mmol})$ and hexamethylbenzene $(0.81$ $\mathrm{mg}, 0.005 \mathrm{mmol})$ as internal standard. $\mathrm{CD}_{2} \mathrm{Cl}_{2}(0.3 \mathrm{~mL})$ was added to the tube, followed by slowly addition of solution of $3 \mathbf{a}$ (6 mg, $0.013 \mathrm{mmol})$ and Phen $(0.9 \mathrm{mg}, 0.005 \mathrm{mmol})$ in $\mathrm{CD}_{2} \mathrm{Cl}_{2}\left((\mathrm{Phen})\left(\mathrm{C}_{6} \mathrm{~F}_{5}\right)_{3} \mathrm{PAu}(\mathrm{I}) \mathrm{NTf}_{2}\right.$ is insoluble in the $\left.\mathrm{CD}_{2} \mathrm{Cl}_{2}\right)$. The reaction was monitored by ${ }^{1} \mathrm{H}$ and ${ }^{31} \mathrm{P}$ NMR spectroscopy. 
${ }^{1} \mathrm{H}$ NMR $\left(\mathrm{CD}_{2} \mathrm{Cl}_{2}\right)$ spectrum of oxidative addition to $(\mathrm{Phen})\left(\mathrm{C}_{6} \mathrm{~F}_{5}\right)_{3} \mathrm{PAu}^{\mathrm{I}} \mathrm{NTf}_{2}$

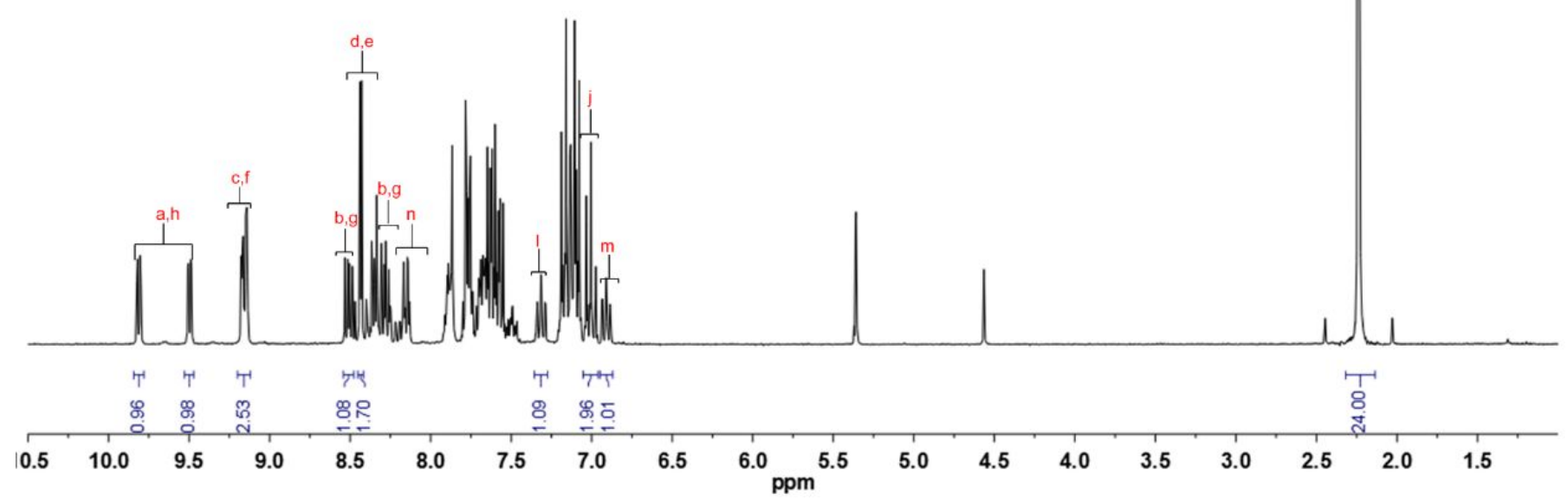

${ }^{31} \mathbf{P}$ NMR $\left(\mathrm{CD}_{2} \mathrm{Cl}_{2}\right)$ spectrum of oxidative addition to $(\mathrm{Phen})\left(\mathrm{C}_{6} \mathrm{~F}_{5}\right)_{3} \mathrm{PAu}^{\mathrm{I}} \mathrm{NTf}_{2}$
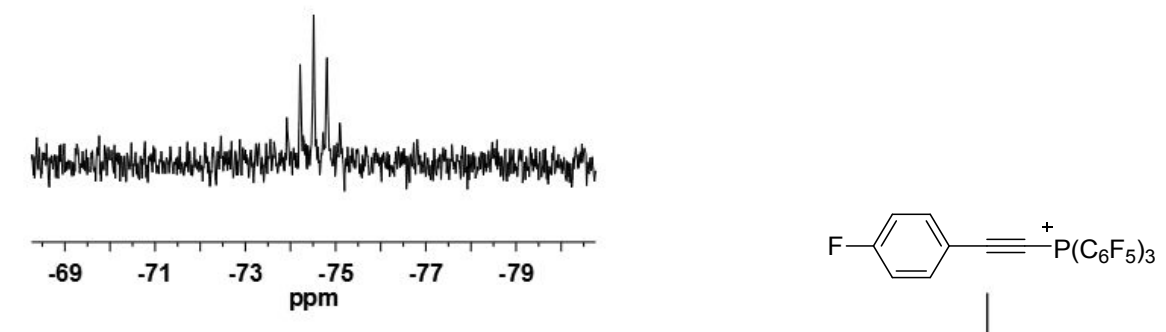

$$
\text { ppm }
$$

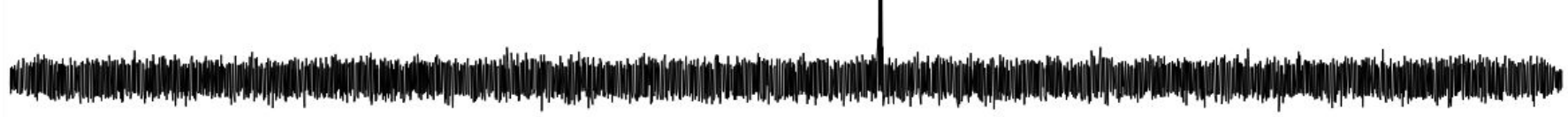

\begin{tabular}{|c|c|c|c|c|c|c|c|c|c|c|c|c|c|}
\hline 140 & 110 & 80 & 60 & 40 & 20 & 0 & -20 & $\begin{array}{c}-50 \\
\mathrm{ppm}\end{array}$ & -80 & -110 & -140 & -180 & -220 \\
\hline
\end{tabular}




\section{Kinetic experiments}

\section{Ligand effects on oxidative addition}

In a glovebox, a J. Young NMR tube was charged with $\mathrm{R}_{3} \mathrm{PAuNTf}_{2}(0.01 \mathrm{mmol})$ and ligand $(0.01 \mathrm{mmol}) \cdot \mathrm{CD}_{2} \mathrm{Cl}_{2}(0.5 \mathrm{~mL})$ was added to the tube, followed by addition of $3 \mathbf{a}(12.2 \mathrm{mg}, 0.025 \mathrm{mmol})$ and hexamethylbenzene $(1.6 \mathrm{mg}, 0.01 \mathrm{mmol})$ as an internal standard. The NMR tube was gently shaken and put into the Avance-III-300 spectrometer immediately. Plots of the yield of $4 \mathbf{a}$ vs time were built.

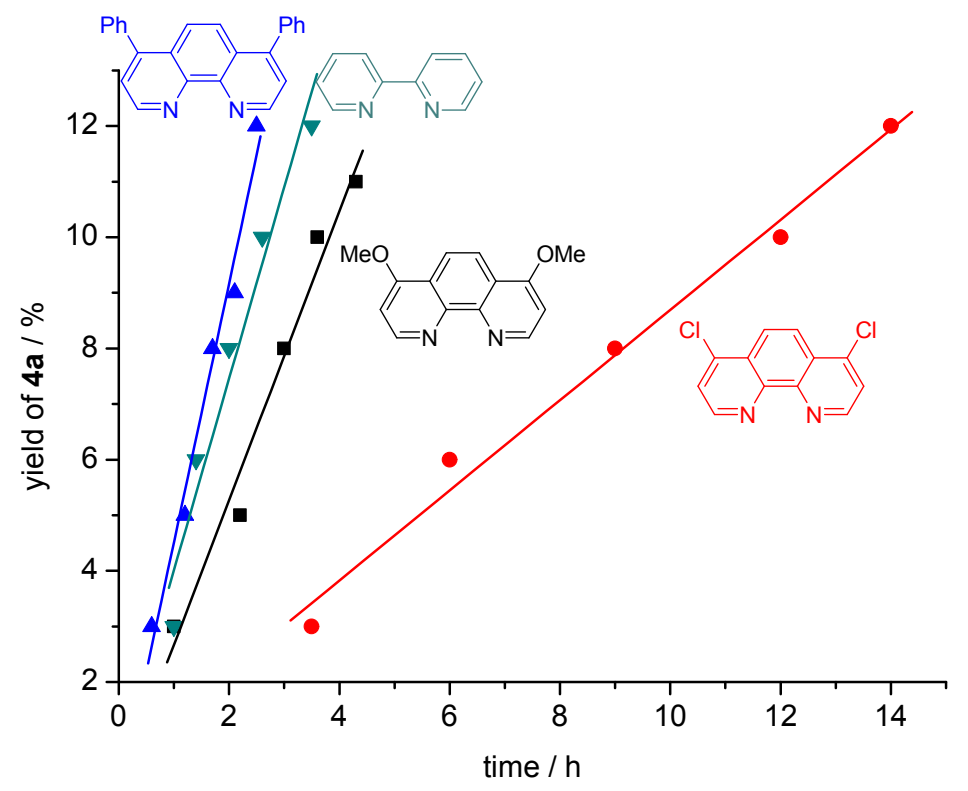

Figure S-2. Effects of bipyridyl ligands for the oxidative addition of alkynyl hypervalent iodine reagent to gold(I).

\section{Solvent effect on oxidative addition}

In a glovebox, a J. Young NMR tube was charged with $\mathrm{Ph}_{3} \mathrm{PAuNTf}_{2}(14.8 \mathrm{mg}, 0.02 \mathrm{mmol})$ and Phen (3.6 mg, $\left.0.02 \mathrm{mmol}\right)$. $\mathrm{CD}_{2} \mathrm{Cl}_{2}$ or $\mathrm{CD}_{3} \mathrm{CN}(0.5 \mathrm{~mL})$ was added to the tube, followed by addition of $3 \mathbf{a}(25 \mathrm{mg}, 0.05 \mathrm{mmol})$ and hexamethylbenzene ( $3.9 \mathrm{mg}, 0.024 \mathrm{mmol}$ ) as an internal standard. The NMR tube was gently shaken and put into the Avance-III-300 spectrometer immediately. Plots of the yield of $\mathbf{4 a}$ vs time were built.

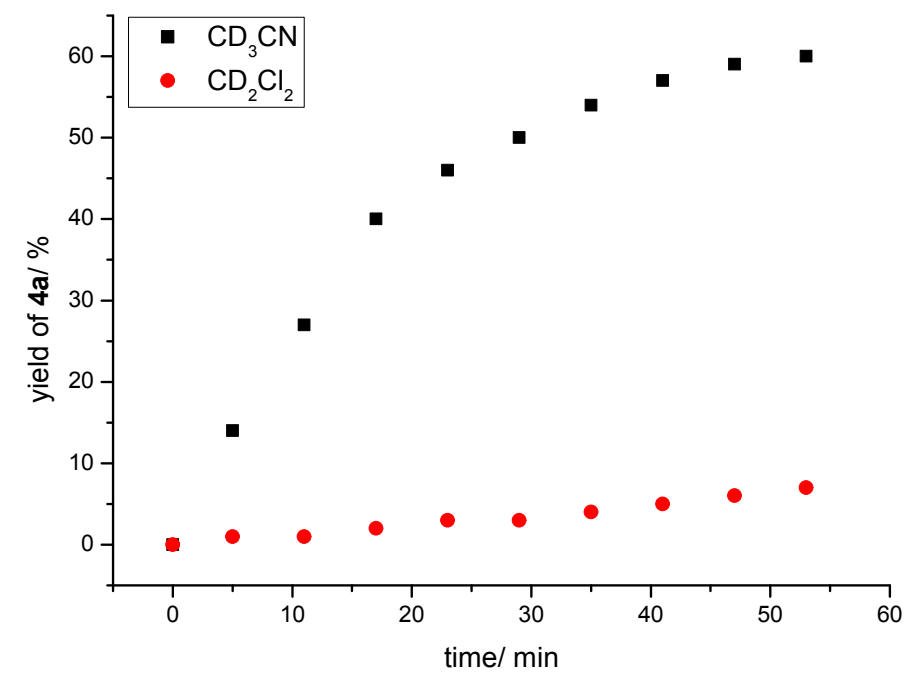




\section{Oxidative addition to linear bis(pyridine)gold(I) complex}

DMSAuCl (60 mg, $0.2 \mathrm{mmol}$ ) was dissolved in DCM (5 M), p-R-pyridine (0.4 mmol) and AgNTf 2 (78 mg, $0.2 \mathrm{mmol})$ was added. After 1 hour of stirring in the dark, the resulting solution was collected by filtration over Celite. The crystalline ( $p$-Rpyridyl $)_{2} \mathrm{AuNTf}_{2}$ was obtained after removing the solvent to $0.5 \mathrm{~mL}$, followed by addition of $2 \mathrm{~mL}$ of $\mathrm{Et}_{2} \mathrm{O}$.

Synthesis of bis(pyridine)gold(I) complex 6a

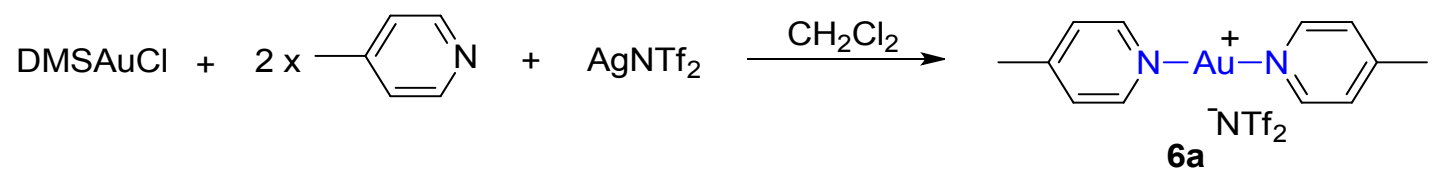

M.p. $161-162{ }^{\circ} \mathrm{C} . \quad{ }^{1} \mathbf{H}$ NMR $\left(301 \mathrm{MHz}, \mathrm{CD}_{2} \mathrm{Cl}_{2}\right) \delta 8.49(\mathrm{dd}, J=5.1,1.5 \mathrm{~Hz}, 4 \mathrm{H}), 7.48(\mathrm{~d}, J=6.0 \mathrm{~Hz}, 4 \mathrm{H}), 2.52(\mathrm{~s}, 6 \mathrm{H}) .{ }^{13} \mathbf{C}$ NMR $\left(126 \mathrm{MHz}, \mathrm{CD}_{2} \mathrm{Cl}_{2}\right) \delta 155.16$ (s), 151.98 (d), 128.30 (d), 120.32 (q: $\left.J=321.8 \mathrm{~Hz}\right), 21.86$ (q). ${ }^{19} \mathbf{F} \mathbf{N M R}\left(471 \mathrm{MHz}, \mathrm{CD}_{2} \mathrm{Cl}_{2}\right) \delta-79.46$ (NTf $)$. IR (ATR): $v\left(\mathrm{~cm}^{-1}\right)=1738,1626,1508,1453,1351,1190,1141,1057,820,790,764,742,718,651,616$. HRMS [ESI, $\left.(\mathrm{M}-\mathrm{NTf})_{2}\right)^{+}$]: $\mathrm{m} / \mathrm{z}$ calcd. for $\mathrm{C}_{12} \mathrm{H}_{14} \mathrm{AuN}_{2}$ 383.0823, found 383.0825 .

Synthesis of bis(pyridine)gold(I) complex $6 \mathrm{~b}$
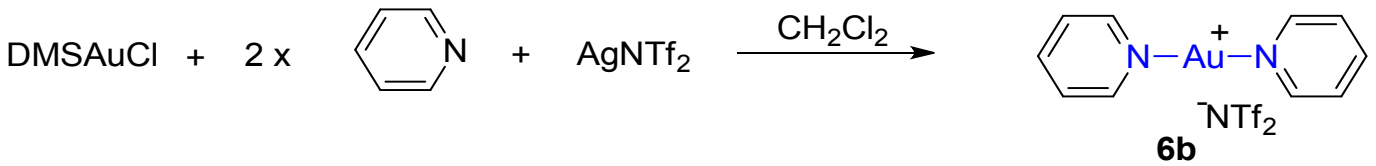

M.p. $108-109^{\circ} \mathrm{C} .{ }^{1} \mathbf{H}$ NMR $\left(500 \mathrm{MHz}, \mathrm{CD}_{2} \mathrm{Cl}_{2}\right) \delta 8.73-8.64(\mathrm{~m}, 4 \mathrm{H}), 8.15(\mathrm{tt}, J=7.9,1.6 \mathrm{~Hz}, 2 \mathrm{H}), 7.70(\mathrm{ddd}, J=7.8,5.1,1.4$ $\mathrm{Hz}, 4 \mathrm{H}){ }^{13} \mathbf{C}$ NMR $\left(126 \mathrm{MHz}, \mathrm{CD}_{2} \mathrm{Cl}_{2}\right) \delta 153.01$ (d), 142.09 (s), 127.71 (d), 120.31 (q: $\left.J=321.4 \mathrm{~Hz}, \mathrm{CF}_{3}\right) .{ }^{19} \mathbf{F} \mathbf{~ N M R}(471 \mathrm{MHz}$, $\left.\mathrm{CD}_{2} \mathrm{Cl}_{2}\right) \delta-79.45\left(\mathrm{NTf}_{2}\right)$. IR (ATR): $v\left(\mathrm{~cm}^{-1}\right)=3114,3060,3043,1936,1613,1489,1460,1346,1332,1240,1221,1179$, 1136, 1083, 1053, 1021, 788, 758, 740, 692, 608. HRMS [ESI, $\left.\left(\mathrm{M}-\mathrm{NTf}_{2}\right)^{+}\right]$: $\mathrm{m} / \mathrm{z}$ calcd. for $\mathrm{C}_{10} \mathrm{H}_{10} \mathrm{AuN}_{2} 355.0510$, found 355.0515 .

Synthesis of bis(pyridine)gold(I) complex 6c

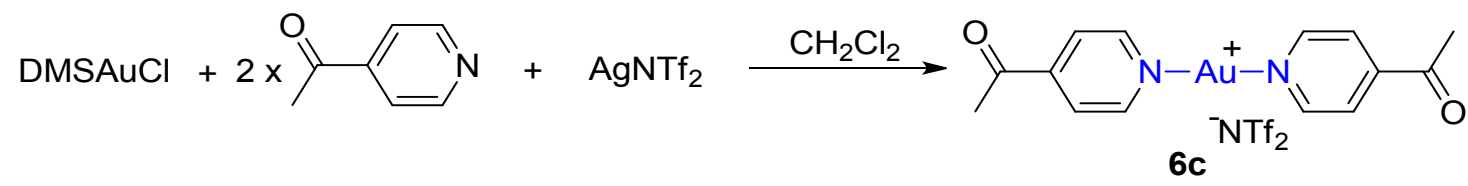

M.p. $120^{\circ} \mathrm{C}$ (decomposed). ${ }^{1} \mathbf{H}$ NMR $\left(500 \mathrm{MHz}, \mathrm{CD}_{2} \mathrm{Cl}_{2}\right) \delta 8.92(\mathrm{dd}, J=5.1,1.6 \mathrm{~Hz}, 4 \mathrm{H}), 8.10(\mathrm{dd}, J=5.1,1.6 \mathrm{~Hz}, 4 \mathrm{H}), 2.71(\mathrm{~s}$, 6H). ${ }^{13} \mathbf{C}$ NMR (126 MHz, $\mathrm{CD}_{2} \mathrm{Cl}_{2}$ ) $\delta 195.32$ (s), 154.30 (d), 146.48 (s), 125.30 (d), 27.07 (q). ${ }^{19} \mathbf{F}$ NMR $\left(471 \mathrm{MHz}, \mathrm{CD}_{2} \mathrm{Cl}_{2}\right) \delta$ $79.36\left(\mathrm{NTf}_{2}\right)$. IR (ATR): $v\left(\mathrm{~cm}^{-1}\right)=3116,3068,3028,1701,1621,1557,1431,1346,1329,1259,1221,1186,1138,1054$, 968, 833, 792, 763, 742, 661, 609. HRMS [ESI, $\left.(\mathrm{M}-\mathrm{NTf})^{+}\right)^{+}$: $\mathrm{m} / \mathrm{z}$ calcd. for $\mathrm{C}_{14} \mathrm{H}_{14} \mathrm{AuN}_{2} \mathrm{O}_{2} 439.0721$, found 439.0723.

Synthesis of bis(pyridine)gold(I) complex 6d

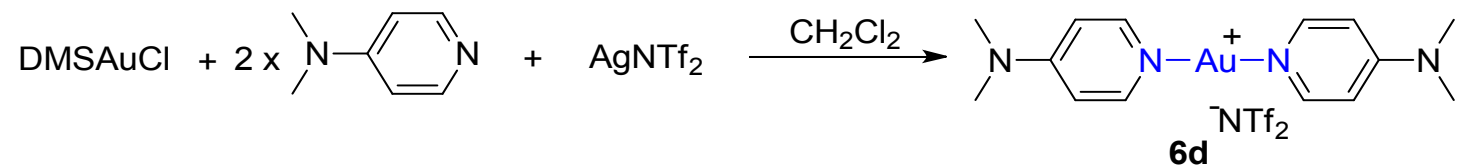

M.p. $159^{\circ} \mathrm{C}$ (decomposed). ${ }^{1} \mathrm{H}$ NMR $\left(500 \mathrm{MHz}, \mathrm{CD}_{2} \mathrm{Cl}_{2}\right) \delta 8.02(\mathrm{dd}, J=5.9,1.4 \mathrm{~Hz}, 4 \mathrm{H}), 6.60(\mathrm{dd}, J=5.9,1.5 \mathrm{~Hz}, 4 \mathrm{H}), 3.11(\mathrm{~s}$, 12H). ${ }^{13} \mathbf{C}$ NMR (126 MHz, $\left.\mathrm{CD}_{2} \mathrm{Cl}_{2}\right) \delta 155.58$ (s), 150.96 (d), 120.34 (q: $\left.J=321.5 \mathrm{~Hz}\right), 108.28$ (d), 39.72 (q). ${ }^{19} \mathbf{F}$ NMR (471 MHz, $\left.\mathrm{CD}_{2} \mathrm{Cl}_{2}\right) \delta-79.48\left(\mathrm{NTf}_{2}\right)$. IR (ATR): $v\left(\mathrm{~cm}^{-1}\right)=2938,2598,1738,1620,1547,1445,1393,1350,1334,1227,1178,1137$, 1083, 1056, 1034, 948, 807, 761, 739, 611. HRMS [ESI, $\left.\left(\mathrm{M}-\mathrm{NTf}_{2}\right)^{+}\right]: \mathrm{m} / \mathrm{z}$ calcd. for $\mathrm{C}_{14} \mathrm{H}_{20} \mathrm{AuN}_{4} 441.1353$, found 441.1350. 


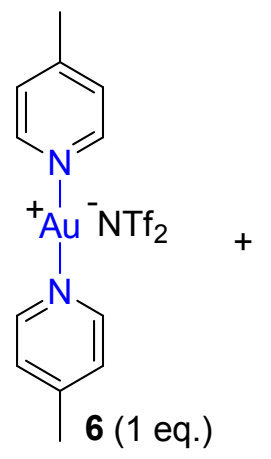<smiles>FC(F)(F)C1(C(F)(F)F)OI(C#CPc2ccccc2)c2ccccc21</smiles>

$3 a$ (1 eq.)

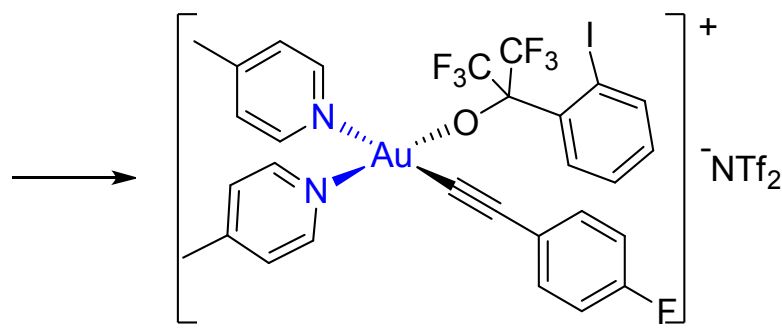

7a $76 \%$ NMR yield

In a glovebox, a J. Young NMR tube was charged with ( $p$-Me-pyridyl) ${ }_{2} \mathrm{AuNTf}_{2}(6.6 \mathrm{mg}, 0.01 \mathrm{mmol}), 3 \mathrm{a}(4.9 \mathrm{mg}, 0.01 \mathrm{mmol})$. $\mathrm{CD}_{2} \mathrm{Cl}_{2}(0.5 \mathrm{~mL})$ was added to the tube. The reaction was monitored by ${ }^{1} \mathrm{H}$ and ${ }^{19} \mathrm{~F}$ NMR spectroscopy and ESI-Ms spectroscopy. Hexamethylbenzene was used as an internal standard.

${ }^{1}$ H NMR $\left(301 \mathrm{MHz}, \mathrm{CD}_{2} \mathrm{Cl}_{2}\right) \delta 9.02(\mathrm{~d}, J=6.8 \mathrm{~Hz}, 2 \mathrm{H}), 8.20(\mathrm{dd}, J=8.1,0.9 \mathrm{~Hz}, 1 \mathrm{H}), 8.14(\mathrm{~d}, J=6.4 \mathrm{~Hz}, 2 \mathrm{H}), 7.85(\mathrm{~d}, J=6.5$ $\mathrm{Hz}, 2 \mathrm{H}), 7.82-7.73(\mathrm{~m}, 2 \mathrm{H}), 7.66(\mathrm{td}, J=7.5,1.0 \mathrm{~Hz}, 1 \mathrm{H}), 7.33(\mathrm{~d}, J=5.9 \mathrm{~Hz}, 2 \mathrm{H}), 7.22(\mathrm{dd}, J=8.8,5.1 \mathrm{~Hz}, 2 \mathrm{H}), 7.06(\mathrm{t}, J=8.6$ $\mathrm{Hz}, 2 \mathrm{H}), 2.69$ (s, 3H), $2.42(\mathrm{~s}, 3 \mathrm{H}) .{ }^{19} \mathbf{F}$ NMR $\left(283 \mathrm{MHz}, \mathrm{CD}_{2} \mathrm{Cl}_{2}\right) \delta-76.22,-108.28$. HRMS [ESI, $\left.(\mathrm{M}-\mathrm{NTf})^{+}\right]$: m/z calcd. for $\mathrm{C}_{29} \mathrm{H}_{22} \mathrm{AuF}_{7} \mathrm{IN}_{2} \mathrm{O} 871.0325$, found 871.0337 .

\section{Synthesis of gold(III) complex $7 \mathrm{~b}$}

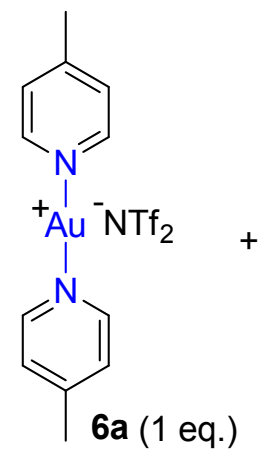<smiles>CC1(C(F)(F)F)OI(C#Cc2ccccc2)c2ccccc21</smiles>

$3 \mathbf{b}$ (1 eq.)<smiles></smiles>

7b $78 \%$ NMR yield

${ }^{1} \mathbf{H}$ NMR $\left(301 \mathrm{MHz}, \mathrm{CD}_{2} \mathrm{Cl}_{2}\right) \delta 9.01(\mathrm{~d}, J=6.8 \mathrm{~Hz}, 2 \mathrm{H}), 8.22(\mathrm{dd}, J=8.1,0.9 \mathrm{~Hz}, 1 \mathrm{H}), 8.13(\mathrm{~d}, J=6.6 \mathrm{~Hz}, 2 \mathrm{H}), 7.85(\mathrm{~d}, J=6.4$ $\mathrm{Hz}, 2 \mathrm{H}), 7.83-7.73(\mathrm{~m}, 2 \mathrm{H}), 7.67(\mathrm{dd}, J=7.4,1.0 \mathrm{~Hz}, 1 \mathrm{H}), 7.46-7.37$ (m, 3H), $7.34(\mathrm{~d}, J=6.1 \mathrm{~Hz}, 2 \mathrm{H}), 7.24-7.18(\mathrm{~m}, 2 \mathrm{H})$, $2.69(\mathrm{~s}, 3 \mathrm{H}), 2.42(\mathrm{~s}, 3 \mathrm{H}) .{ }^{19} \mathbf{F}$ NMR $\left(283 \mathrm{MHz}, \mathrm{CD}_{2} \mathrm{Cl}_{2}\right) \delta-76.24\left(\mathrm{~s}, \mathrm{CF}_{3}\right),-79.29\left(\mathrm{~s}, \mathrm{NTf}_{2}\right) . \mathbf{H R M S}\left[\mathrm{ESI},(\mathrm{M}-\mathrm{NTf})^{+}\right]: \mathrm{m} / \mathrm{z} \mathrm{calcd}$. for $\mathrm{C}_{29} \mathrm{H}_{23} \mathrm{AuF}_{6} \mathrm{IN}_{2} \mathrm{O}$ 853.0425, found 853.0415.

Synthesis of gold(III) complex 7c<smiles></smiles><smiles>Cc1ccccc1C#CI1OC(C(F)(F)F)(C(F)(F)F)c2ccccc21</smiles>

$3 c$ (1 eq.)

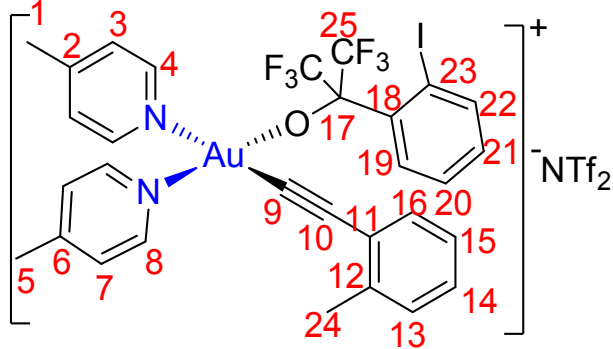

7c $82 \%$ NMR yield 
${ }^{1}$ H NMR $\left(600 \mathrm{MHz}, \mathrm{CD}_{2} \mathrm{Cl}_{2}\right) \delta 9.07$ (d, $J=6.6 \mathrm{~Hz}, \mathrm{H} 4$ or H8), 8.23 (d, $\left.J=8.4 \mathrm{~Hz}, \mathrm{H} 22\right), 8.09$ (d, $\left.J=6.0 \mathrm{~Hz}, \mathrm{H} 4 \mathrm{or} \mathrm{H8}\right), 7.77$ 7.67 (m, H3 or H7, H19 and H21), 7.57 (t, $J=7.5 \mathrm{~Hz}, \mathrm{H} 20), 7.28-7.24$ (m, H3 or H7, and H14), 7.18 (d, $J=7.3 \mathrm{~Hz}, \mathrm{H} 16$ ), 7.13 (t, $J=7.8 \mathrm{~Hz}, \mathrm{H13}$ and H15), 2.57 (s, H), 2.34 (s, H), 2.01 (s, H). ${ }^{13} \mathbf{C}$ NMR (151 MHz, $\left.\mathrm{CD}_{2} \mathrm{Cl}_{2}\right) \delta 161.72$ (C2 or C6), 153.84 (C2 or C6), 150.79 (C4 or C8), 144.30 (C18), 142.50 (C4 or C8), 136.99 (C12), 135.96 (C11), 132.75 (C19 or C21), 132.00 (C13), 131.58 (C14), 131.50 (C10), 131.18 (C16), 130.78 (C20), 130.27 (C19 or C21), 129.26 (C22), 128.50 (C3 or C7), 127.46 (C3 or C7), 127.31 (C15), 125.44 (C9 or C10), 123.52 (C9 or C10), 120.20 (q, $J_{C-F}=321.6 \mathrm{~Hz}, \mathrm{CF}_{3}$ ), 113.35 (C23), $82.37-80.80$ (m, C17), 22.62 (C1 or C5), 21.59 (C1 or C5), 19.81 (C24). ${ }^{19} \mathbf{F}$ NMR $\left(283 \mathrm{MHz}, \mathrm{CD}_{2} \mathrm{Cl}_{2}\right) \delta-75.87\left(\mathrm{q}, J_{F-F}=8.7 \mathrm{~Hz}, \mathrm{CF}_{3}\right),-76.56(\mathrm{q}$, $\left.J_{F-F}=8.7 \mathrm{~Hz}, \mathrm{CF}_{3}\right),-79.29(\mathrm{~s})$. HRMS [ESI, $\left.\left(\mathrm{M}-\mathrm{NTf}_{2}\right)^{+}\right]: \mathrm{m} / \mathrm{z}$ calcd. for $\mathrm{C}_{30} \mathrm{H}_{25} \mathrm{AuF}_{6} \mathrm{IN}_{2} \mathrm{O}$ 867.0576, found 867.0591.

\section{Synthesis of gold(III) complex 7d}

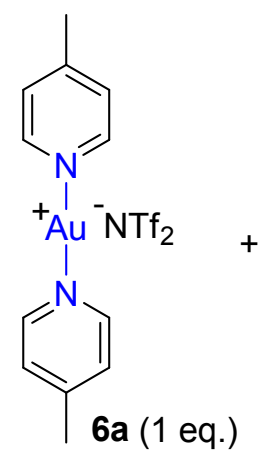

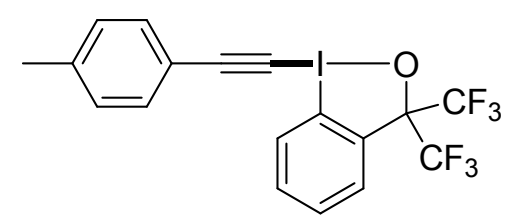

3d (1 eq.)

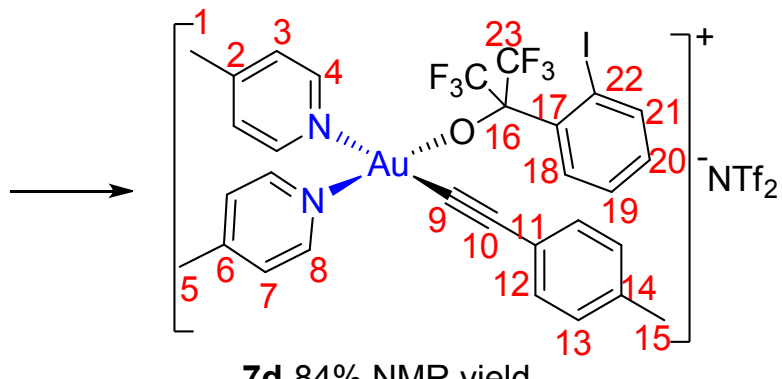

7d $84 \%$ NMR yield

${ }^{1} \mathbf{H}$ NMR $\left(600 \mathrm{MHz}, \mathrm{CD}_{2} \mathrm{Cl}_{2}\right) \delta 9.00(\mathrm{~d}, J=6.7 \mathrm{~Hz}, \mathrm{H} 4$ or H8), 8.23 (dd, $J=8.2,0.6 \mathrm{~Hz}, \mathrm{H} 21), 8.12$ (d, $J=6.5 \mathrm{~Hz}, \mathrm{H} 4$ or H8), 7.84 (d, $J=6.5 \mathrm{~Hz}, \mathrm{H} 3$ or H7), 7.80 (d, $J=7.7 \mathrm{~Hz}, \mathrm{H} 18), 7.78-7.74$ (m, H20), $7.68-7.61$ (m, H19), 7.32 (d, $J=6.1 \mathrm{~Hz}, \mathrm{H} 3$ or H7), 7.18 (d, $J=8.0 \mathrm{~Hz}, \mathrm{H} 12), 7.08$ (d, $J=8.1 \mathrm{~Hz}, \mathrm{H} 13), 2.68$ (s, H1 or H5), 2.41 (s, H1 or H5), 2.34 (s, H15). ${ }^{13} \mathbf{C}$ NMR (151 MHz, $\left.\mathrm{CD}_{2} \mathrm{Cl}_{2}\right) \delta 161.88(\mathrm{~s}), 153.93(\mathrm{~s}), 150.84(\mathrm{~d}), 142.97$ (d), 142.24 (s), 133.39 (s), 132.83 (d), 131.70 (s), 130.83 (d), 130.57 (d), 130.39 (d), 129.50 (d), 129.17 (d), 128.72 (d), 127.54 (d), 125.58 (s), 123.64 (s), 120.30 (q: $\left.J_{C-F}=321.4 \mathrm{~Hz}\right), 113.55$ (s), $82.88-80.77$ (m), 22.75 (q), 21.68 (q), 21.51 (q). ${ }^{19} \mathbf{F}$ NMR (283 MHz, $\left.\mathrm{CD}_{2} \mathrm{Cl}_{2}\right) \delta-76.23\left(\mathrm{CF}_{3}\right),-79.31\left(\mathrm{NTf}_{2}\right)$. HRMS [ESI, $\left.(\mathrm{M}-\mathrm{NTf})^{+}\right]$]: $\mathrm{m} / \mathrm{z}$ calcd. for $\mathrm{C}_{30} \mathrm{H}_{25} \mathrm{AuF}_{6} \mathrm{IN}_{2} \mathrm{O}$ 867.0576, found 867.0585.

\section{Synthesis of gold(III) complex $7 \mathrm{e}$}

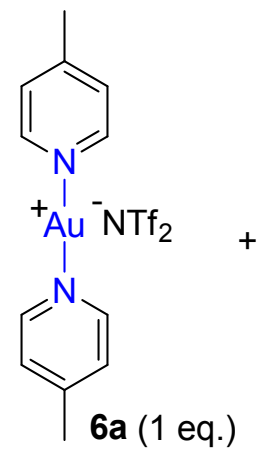

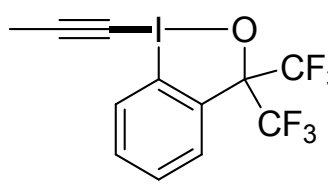

3 e (1 eq.)

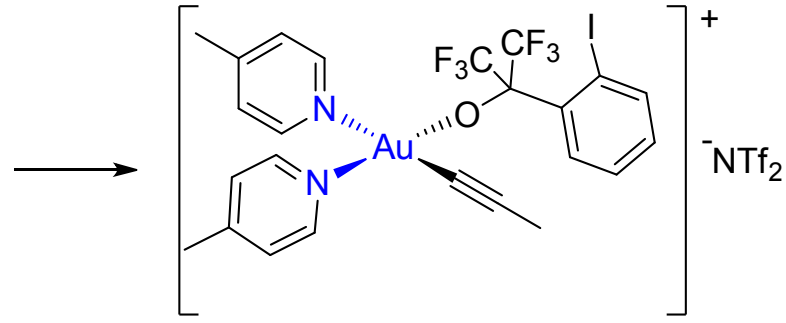

7e $49 \%$ NMR yield

${ }^{1} \mathrm{H}$ NMR $\left(301 \mathrm{MHz}, \mathrm{CD}_{2} \mathrm{Cl}_{2}\right) \delta 8.52(\mathrm{~d}, J=6.7 \mathrm{~Hz}, 2 \mathrm{H}), 8.12(\mathrm{~d}, J=6.5 \mathrm{~Hz}, 2 \mathrm{H}), 7.91(\mathrm{~d}, J=6.3 \mathrm{~Hz}, 2 \mathrm{H}), 7.86(\mathrm{~d}, J=6.3 \mathrm{~Hz}, 1 \mathrm{H})$, $7.79-7.71(\mathrm{~m}, 1 \mathrm{H}), 7.71-7.62(\mathrm{~m}, 2 \mathrm{H}), 7.32(\mathrm{~d}, J=6.0 \mathrm{~Hz}, 2 \mathrm{H}), 2.72(\mathrm{~s}, 3 \mathrm{H}), 2.41(\mathrm{~s}, 3 \mathrm{H}), 2.31(\mathrm{~s}, 3 \mathrm{H}) .{ }^{19} \mathbf{F} \mathbf{~ N M R}(283 \mathrm{MHz}$, $\left.\mathrm{CD}_{2} \mathrm{Cl}_{2}\right) \delta-76.25\left(\mathrm{CF}_{3}\right),-79.32\left(\mathrm{NTf}_{2}\right)$. HRMS [ESI, $\left(\mathrm{M}-\mathrm{NTf}_{2}\right)^{+}$]: m/z calcd. for $\mathrm{C}_{24} \mathrm{H}_{21} \mathrm{AuF}_{6} \mathrm{IN}_{2} \mathrm{O}$ 791.0268, found 791.0260.

\section{Synthesis of gold(III) complex $7 \mathrm{f}$}




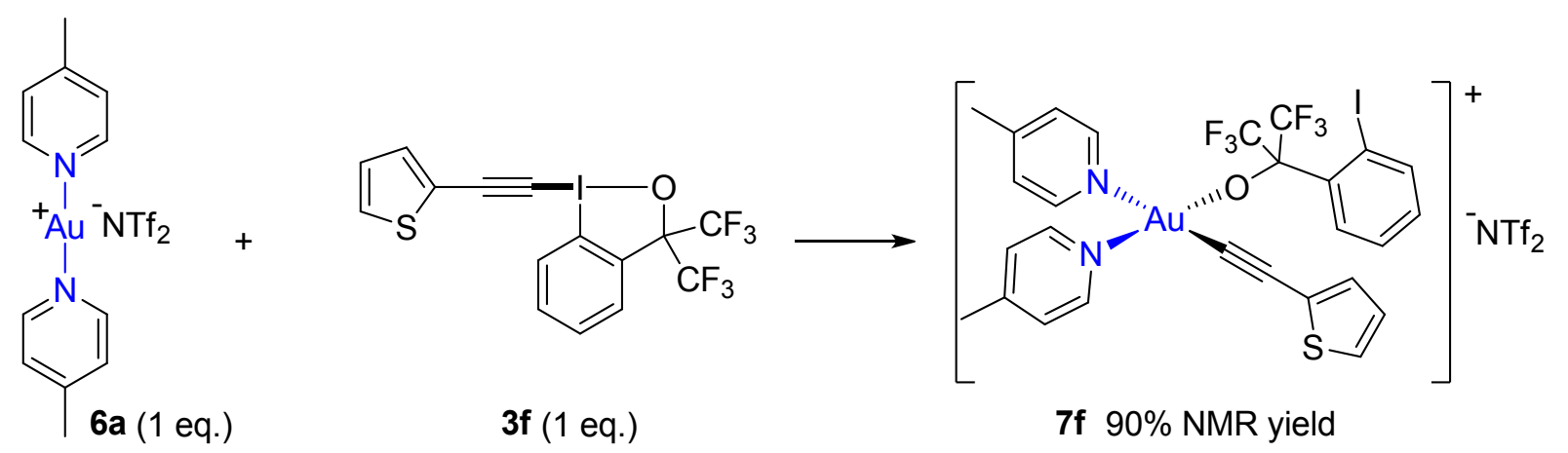

${ }^{1} \mathbf{H}$ NMR $\left(500 \mathrm{MHz}, \mathrm{CD}_{2} \mathrm{Cl}_{2}\right) \delta 9.02(\mathrm{~d}, J=6.8 \mathrm{~Hz}, 2 \mathrm{H}), 8.14-8.07(\mathrm{~m}, 3 \mathrm{H}), 7.88(\mathrm{~d}, J=6.5 \mathrm{~Hz}, 2 \mathrm{H}), 7.82(\mathrm{~d}, J=7.5 \mathrm{~Hz}, 1 \mathrm{H})$, $7.78-7.72(\mathrm{~m}, 1 \mathrm{H}), 7.69-7.64(\mathrm{~m}, 1 \mathrm{H}), 7.49(\mathrm{dd}, J=5.1,1.2 \mathrm{~Hz}, 1 \mathrm{H}), 7.33(\mathrm{~d}, J=6.0 \mathrm{~Hz}, 2 \mathrm{H}), 7.08(\mathrm{dd}, J=3.7,1.2 \mathrm{~Hz}, 1 \mathrm{H})$, $7.04(\mathrm{dd}, J=5.1,3.7 \mathrm{~Hz}, 1 \mathrm{H}), 2.71(\mathrm{~s}, 3 \mathrm{H}), 2.41(\mathrm{~s}, 3 \mathrm{H}) .{ }^{19} \mathbf{F}$ NMR $\left(471 \mathrm{MHz}, \mathrm{CD}_{2} \mathrm{Cl}_{2}\right) \delta-76.20\left(\mathrm{CF}_{3}\right),-79.29(\mathrm{NTf}) . \mathbf{H R M S}[\mathrm{ESI}$, $\left.\left(\mathrm{M}-\mathrm{NTf}_{2}\right)^{+}\right]$: $\mathrm{m} / \mathrm{z}$ calcd. for $\mathrm{C}_{27} \mathrm{H}_{21} \mathrm{AuF}_{6} \mathrm{IN}_{2} \mathrm{OS} 858.9989$, found 858.9972.

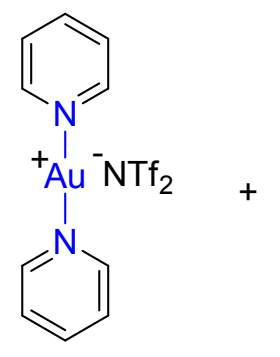

6b (1 eq.)

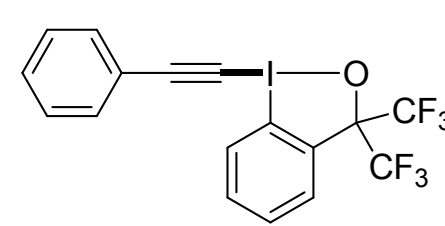

3b (1 eq.)

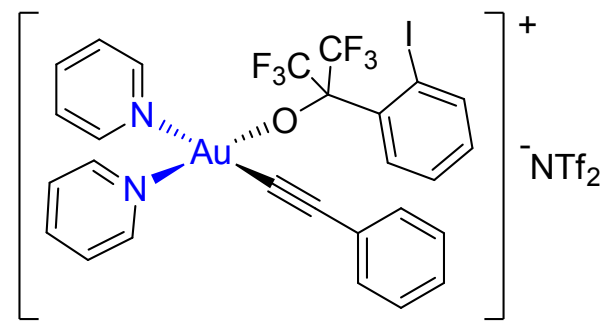

7g $83 \%$ NMR yield

${ }^{1} \mathbf{H}$ NMR $\left(500 \mathrm{MHz}, \mathrm{CD}_{2} \mathrm{Cl}_{2}\right) \delta 9.24(\mathrm{dd}, J=6.7,1.2 \mathrm{~Hz}, 2 \mathrm{H}), 8.59(\mathrm{tt}, J=7.8,1.3 \mathrm{~Hz}, 1 \mathrm{H}), 8.29(\mathrm{dd}, J=6.4,1.5 \mathrm{~Hz}, 2 \mathrm{H}), 8.24$ $(\mathrm{dd}, J=8.2,0.7 \mathrm{~Hz}, 1 \mathrm{H}), 8.11(\mathrm{dd}, J=7.7,6.8 \mathrm{~Hz}, 2 \mathrm{H}), 7.96(\mathrm{ddd}, J=7.8,4.7,1.6 \mathrm{~Hz}, 1 \mathrm{H}), 7.84-7.76(\mathrm{~m}, 2 \mathrm{H}), 7.70-7.65(\mathrm{~m}$, $1 \mathrm{H}), 7.56-7.52(\mathrm{~m}, 2 \mathrm{H}), 7.47-7.42(\mathrm{~m}, 1 \mathrm{H}), 7.42-7.36(\mathrm{~m}, 2 \mathrm{H}), 7.24(\mathrm{~d}, J=7.2 \mathrm{~Hz}, 2 \mathrm{H}) .{ }^{19} \mathbf{F} \mathbf{N M R}\left(471 \mathrm{MHz} \mathrm{CD}_{2} \mathrm{Cl}_{2}\right) \delta-76.21$ $\left(\mathrm{CF}_{3}\right),-79.29\left(\mathrm{NTf}_{2}\right)$. HRMS [ESI, $\left.\left(\mathrm{M}-\mathrm{NTf}_{2}\right)^{+}\right]$: $\mathrm{m} / \mathrm{z}$ calcd. for $\mathrm{C}_{27} \mathrm{H}_{19} \mathrm{AuF}_{6} \mathrm{IN}_{2} \mathrm{O} 825.0112$, found 825.0125 .
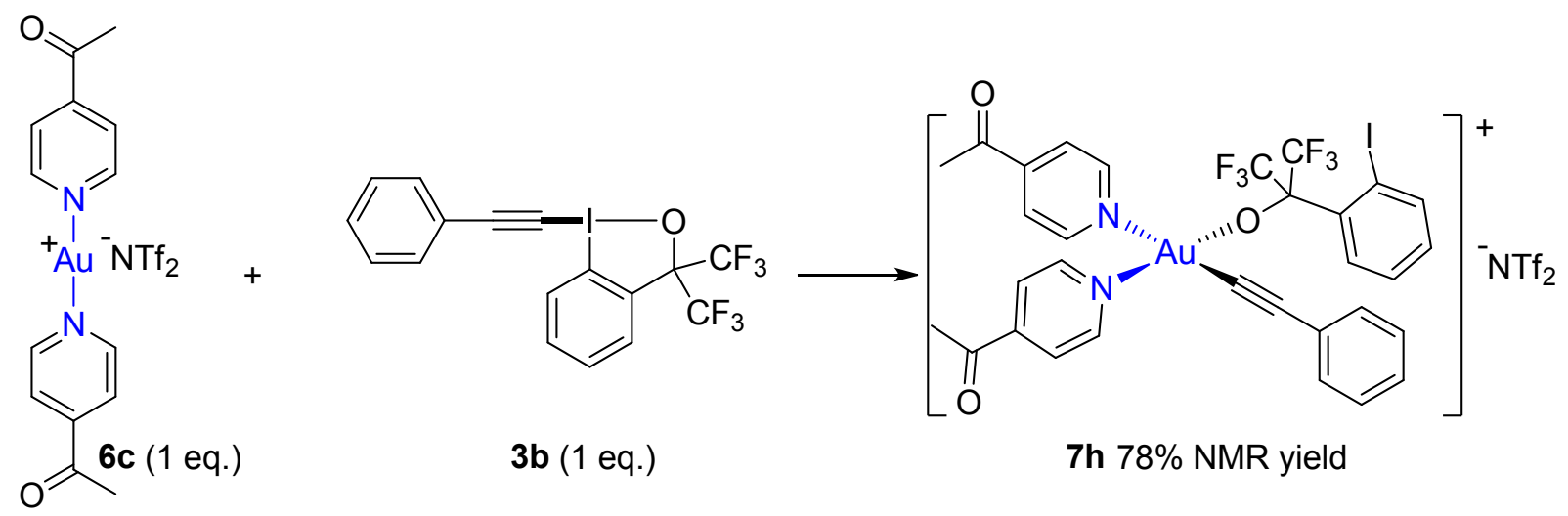

${ }^{1} \mathbf{H}$ NMR $\left(500 \mathrm{MHz}, \mathrm{CD}_{2} \mathrm{Cl}_{2}\right) \delta 9.44(\mathrm{~d}, J=6.8 \mathrm{~Hz}, 2 \mathrm{H}), 8.54(\mathrm{dd}, J=5.1,1.5 \mathrm{~Hz}, 2 \mathrm{H}), 8.44(\mathrm{~d}, J=6.8 \mathrm{~Hz}, 2 \mathrm{H}), 8.27(\mathrm{~d}, J=8.2$ $\mathrm{Hz}, 1 \mathrm{H}), 7.93(\mathrm{dd}, J=5.1,1.5 \mathrm{~Hz}, 2 \mathrm{H}), 7.80(\mathrm{t}, J=7.7 \mathrm{~Hz}, 2 \mathrm{H}), 7.68(\mathrm{t}, J=7.4 \mathrm{~Hz}, 1 \mathrm{H}), 7.49-7.44(\mathrm{~m}, 1 \mathrm{H}), 7.40$ (t, $J=7.7 \mathrm{~Hz}$, 2H), $7.26-7.22(\mathrm{~m}, 2 \mathrm{H}), 2.77(\mathrm{~s}, 3 \mathrm{H}), 2.62(\mathrm{~s}, 3 \mathrm{H}) .{ }^{19} \mathbf{F}$ NMR (471 MHz, $\left.\mathrm{CD}_{2} \mathrm{Cl}_{2}\right) \delta-76.21\left(\mathrm{CF}_{3}\right),-79.18\left(\mathrm{NTf}_{2}\right)$. HRMS [ESI, $\left.\left(\mathrm{M}-\mathrm{NTf}_{2}\right)^{+}\right]: \mathrm{m} / \mathrm{z}$ calcd. for $\mathrm{C}_{31} \mathrm{H}_{23} \mathrm{AuF}_{6} \mathrm{IN}_{2} \mathrm{O}_{3}$ 909.0323, found 909.0328. 


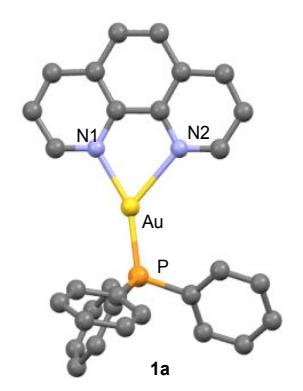

\section{checkCIF/PLATON report}

You have not supplied any structure factors. As a result the full set of tests cannot be run.

THIS REPORT IS FOR GUIDANCE ONLY. IF USED AS PART OF A REVIEW PROCEDURE FOR PUBLICATION, IT SHOULD NOT REPLACE THE EXPERTISE OF AN EXPERIENCED CRYSTALLOGRAPHIC REFEREE.

No syntax errors found. CIF dictionary Interpreting this report

\section{Datablock: yyy8}

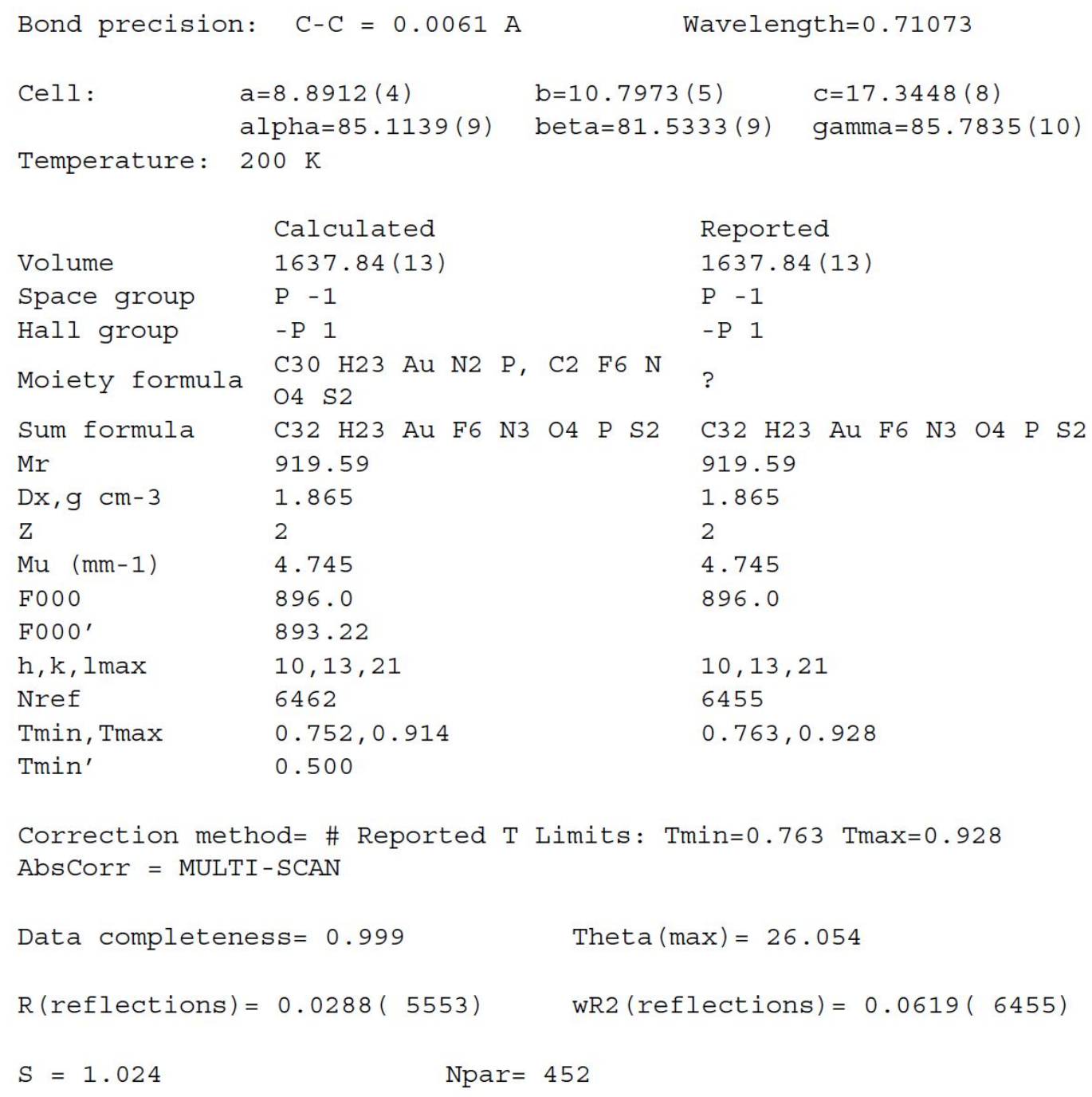




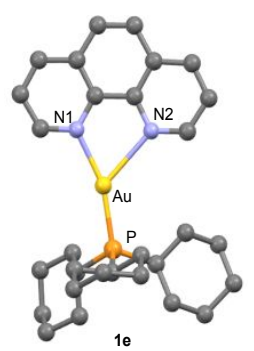




\section{checkCIF/PLATON report}

You have not supplied any structure factors. As a result the full set of tests cannot be run.

THIS REPORT IS FOR GUIDANCE ONLY. IF USED AS PART OF A REVIEW PROCEDURE FOR PUBLICATION, IT SHOULD NOT REPLACE THE EXPERTISE OF AN EXPERIENCED CRYSTALLOGRAPHIC REFEREE.

No syntax errors found. CIF dictionary Interpreting this report

\section{Datablock: yyy11}

\begin{tabular}{llll} 
Bond precision: $\quad \mathrm{C}-\mathrm{C}=0.0047 \mathrm{~A}$ & \multicolumn{1}{c}{ Wavelength=0.71073 } \\
Cell: & $\mathrm{a}=11.1523(5)$ & $\mathrm{b}=13.0973(5)$ & $\mathrm{C}=14.0183(6)$ \\
& $\mathrm{a}$ lpha=102.7857(7) & beta=96.5595(7) & gamma=108.8934(7) \\
Temperature: & $200 \mathrm{~K}$ & &
\end{tabular}

\begin{tabular}{|c|c|c|}
\hline & Calculated & Reported \\
\hline Volume & $1850.49(14)$ & $1850.49(14)$ \\
\hline Space group & $P-1$ & $P-1$ \\
\hline Hall group & $-\mathrm{P} 1$ & $\begin{array}{ll}-\mathrm{P} & 1\end{array}$ \\
\hline Moiety formula & $\begin{array}{l}2(\mathrm{C} 30 \mathrm{H} 41 \mathrm{Au} \mathrm{N} 2 \mathrm{P}), \quad 2(\mathrm{C} 2 \\
\mathrm{F} 6 \mathrm{~N} \quad 4 \quad \mathrm{~S} 2), \mathrm{C} \mathrm{H} 2 \mathrm{Cl} 2\end{array}$ & $\mathrm{cos}$ \\
\hline Sum formula & $\begin{array}{l}\text { C65 H84 Au2 C12 F12 N6 O8 } \\
\text { P2 S4 }\end{array}$ & $\begin{array}{l}\mathrm{C} 32.50 \\
\mathrm{P}\end{array}$ \\
\hline $\mathrm{Mr}$ & 1960.40 & 980.19 \\
\hline$D x, g c m-3$ & 1.759 & 1.759 \\
\hline $\mathrm{Z}$ & 1 & 2 \\
\hline $\mathrm{Mu} \quad(\mathrm{mm}-1)$ & 4.275 & 4.275 \\
\hline F000 & 974.0 & 974.0 \\
\hline Fo00' & 971.51 & \\
\hline$h, k, l \max$ & $14,16,17$ & $14,16,17$ \\
\hline Nref & 7910 & 7909 \\
\hline Tmin, Tmax & $0.698,0.735$ & $0.644,0.694$ \\
\hline Tmin' & 0.635 & \\
\hline \multicolumn{3}{|c|}{$\begin{array}{l}\text { Correction method= \# Reported T Limits: Tmin=0.644 Tmax=0.694 } \\
\text { AbsCorr = MULTI-SCAN }\end{array}$} \\
\hline \multicolumn{3}{|c|}{ Data completeness $=1.000$} \\
\hline \multicolumn{3}{|c|}{$R($ reflections $)=0.0233(7212)$} \\
\hline$S=1.031$ & Npar $=474$ & \\
\hline
\end{tabular}




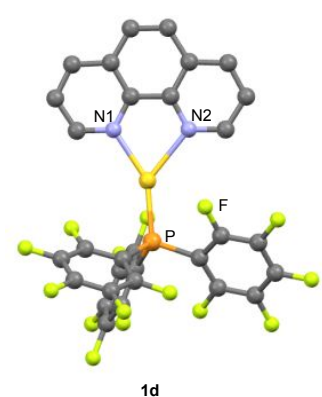




\section{checkCIF/PLATON report}

You have not supplied any structure factors. As a result the full set of tests cannot be run.

THIS REPORT IS FOR GUIDANCE ONLY. IF USED AS PART OF A REVIEW PROCEDURE FOR PUBLICATION, IT SHOULD NOT REPLACE THE EXPERTISE OF AN EXPERIENCED CRYSTALLOGRAPHIC REFEREE.

No syntax errors found. CIF dictionary Interpreting this report

\section{Datablock: yyy12}

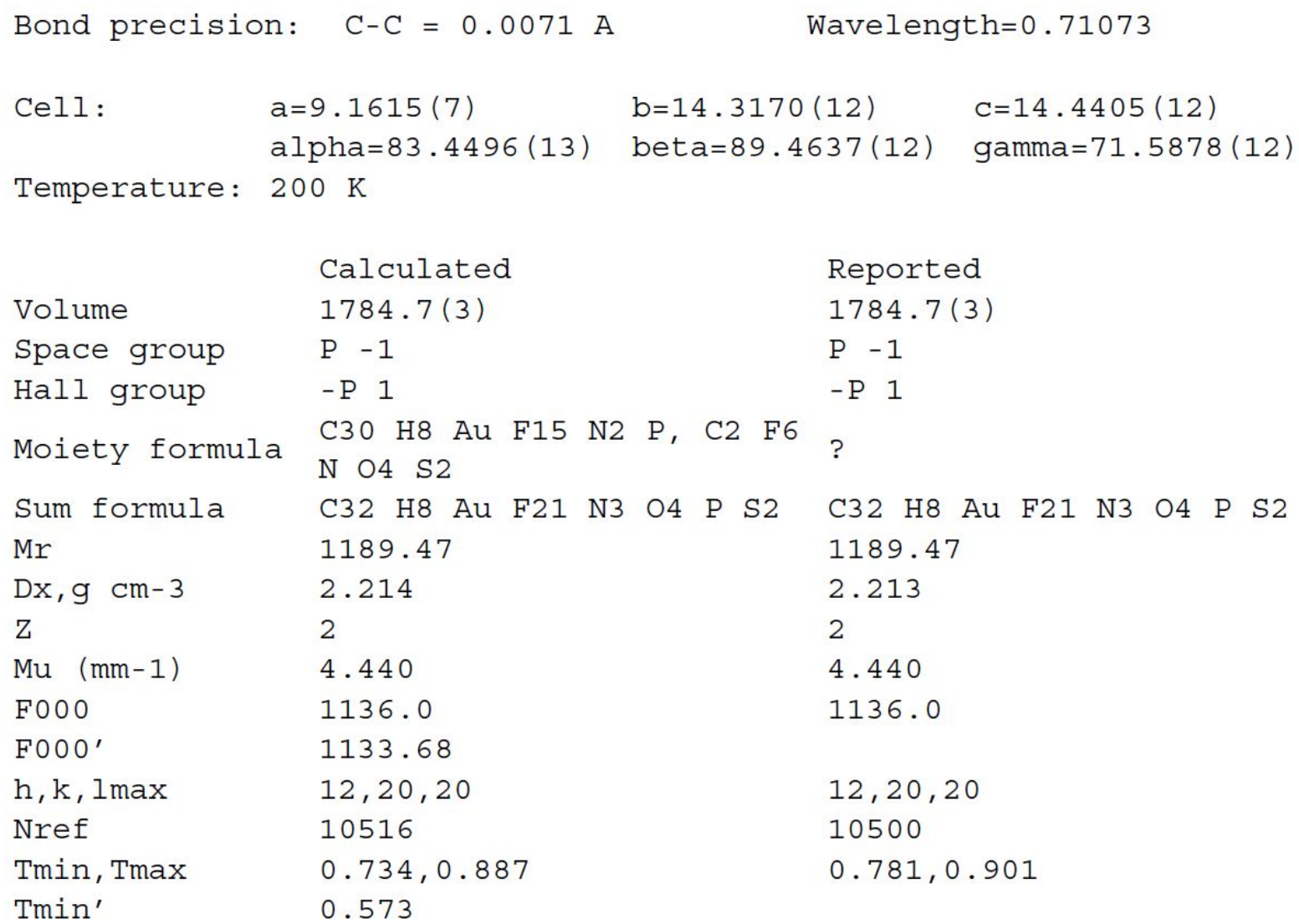

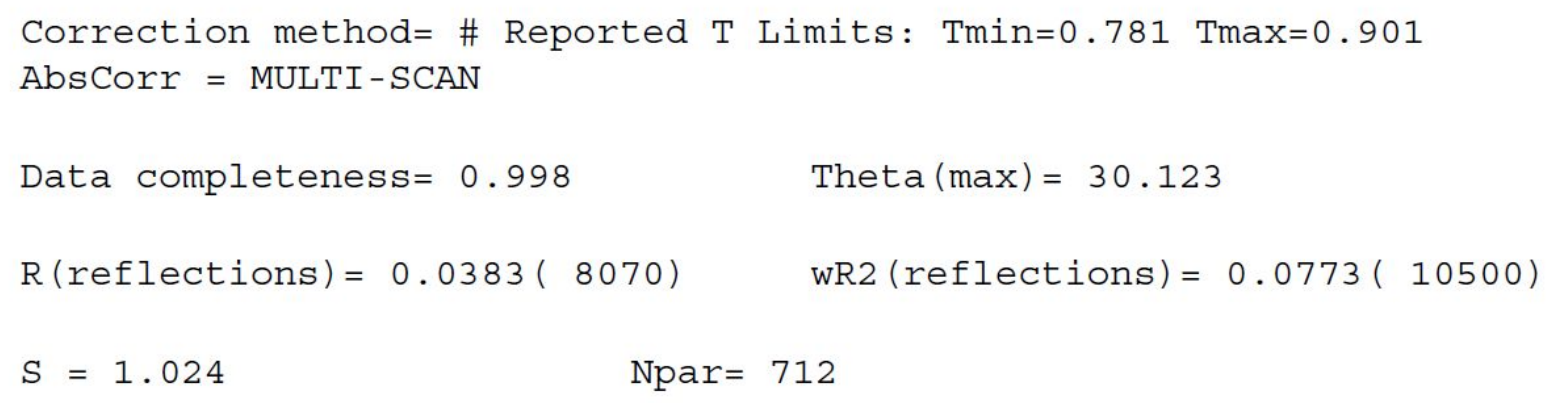

\section{References}

(1) Li, X.; Xie, X.; Sun, N.; Liu, Y. Angew. Chem. Int. Ed. 2017, 56, 6994.

(2) Yang, Y.; Antoni, P.; Zimmer, M.; Sekine, K.; Mulks, F.; Hu, L.; Zhang, L.; Rudolph, M.; Rominger, F.; Hashmi, A. S. 
K. Angew. Chem. Int. Ed. 2019, 58, 5129.

(3) Narbe, M.; Martin H-G. Phys. Chem. Chem. Phys. 2014, 16, 9904.

(4) Weigend, F.; Ahlrichs, R. Phys. Chem. Chem. Phys. 2005, 7, 3297.

(5) Andrae, D.; Haeussermann, U.; Dolg, M.; Stoll, H.; Preuss, H. Theor. Chim. Acta. 1990, 77, 123.

(6) Neese, F. The ORCA program system, Wiley Interdiscip. Rev.: Comput. Mol. Sci. 2012, 2, 73.

\section{NMR spectrum}


${ }^{1} \mathbf{H}$ NMR $\left(400 \mathrm{MHz}, \mathrm{CD}_{2} \mathrm{Cl}_{2}\right)$ spectrum of $\mathbf{1 a}$

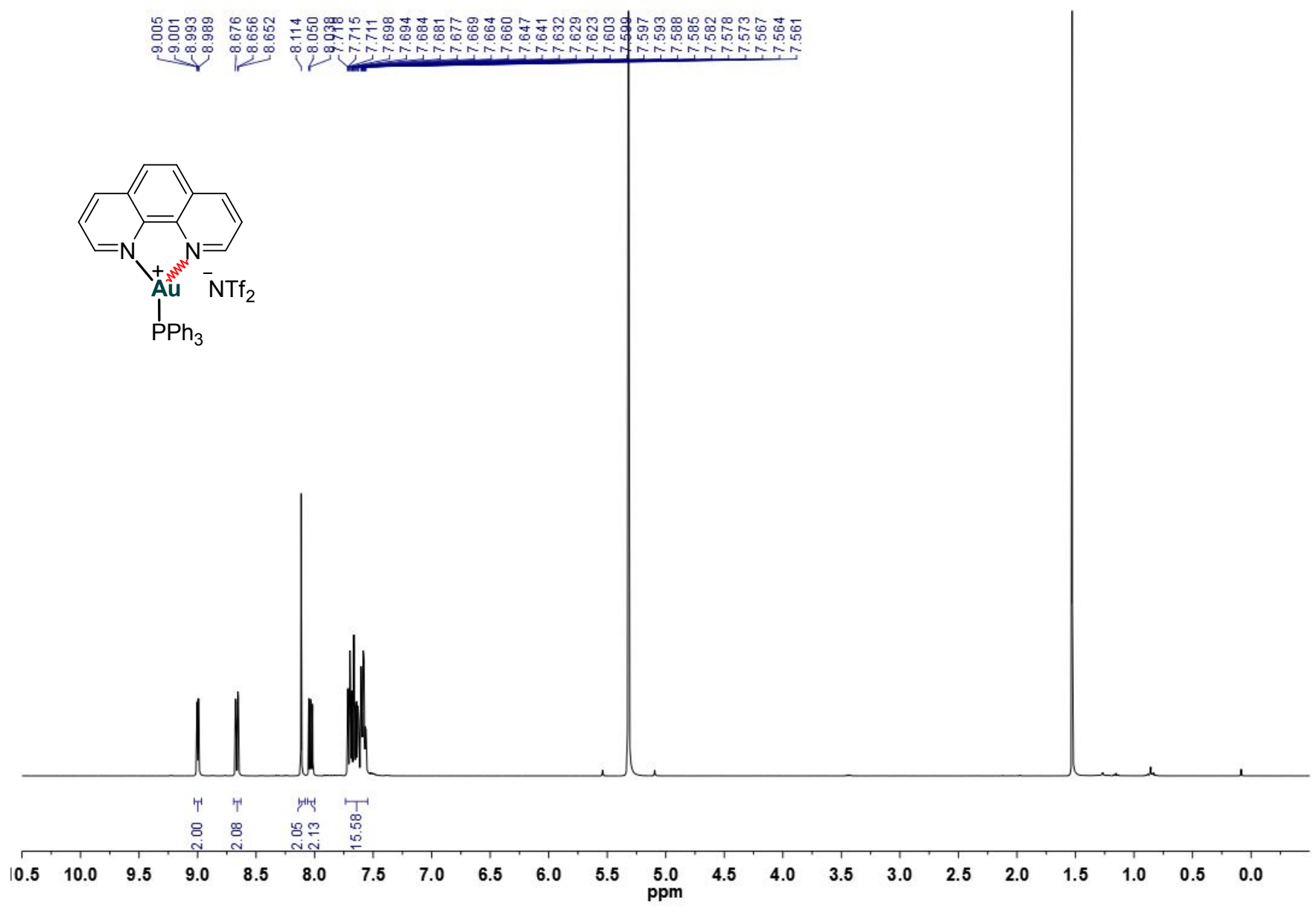

${ }^{13} \mathbf{C ~ N M R}\left(101 \mathrm{MHz}, \mathrm{CD}_{2} \mathrm{Cl}_{2}\right)$ spectrum of $\mathbf{1 a}$ 


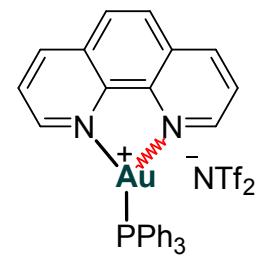

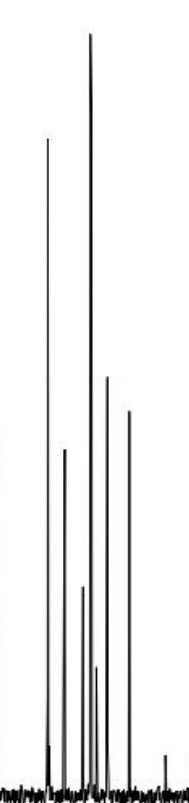

\begin{tabular}{rrrrrrrrrrrrrrrrrrrr}
\hline 180 & 170 & 160 & 150 & 140 & 130 & 120 & 110 & 100 & $\begin{array}{l}90 \\
\text { ppm }\end{array}$ & 80 & 70 & 60 & 50 & 40 & 30 & 20 & 10 & 0
\end{tabular}

${ }^{31} \mathbf{P}$ NMR (122 MHz, CD2Cl2) spectrum of 1a 


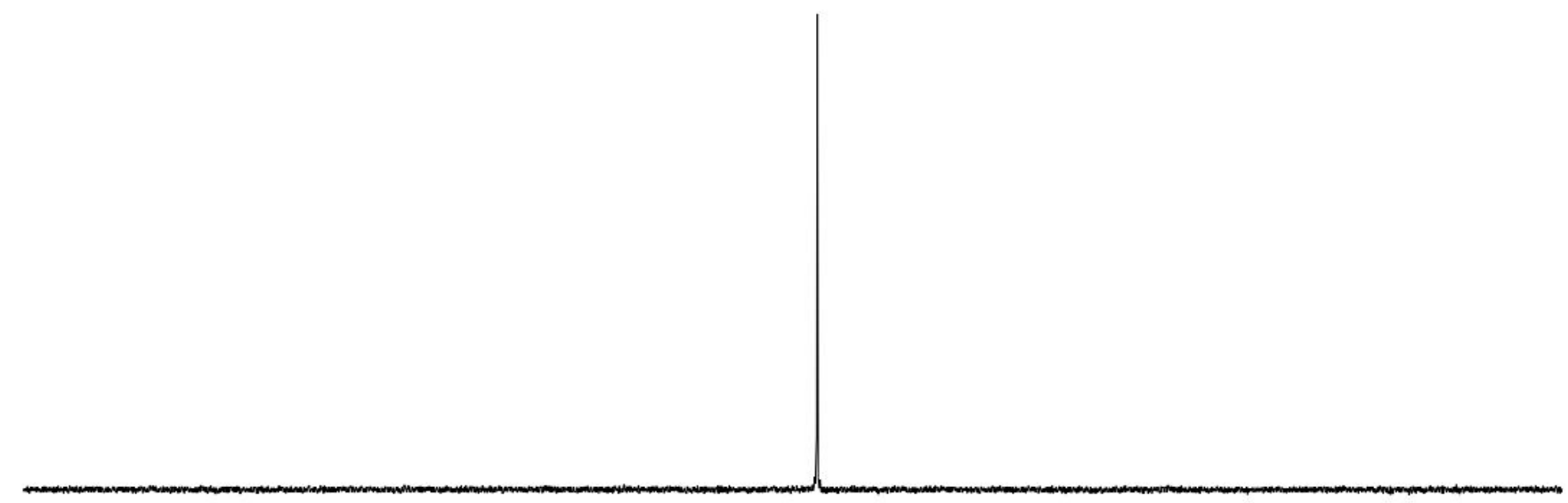

$\begin{array}{llllllllllllllllllllllllllllll}95 & 90 & 85 & 80 & 75 & 70 & 65 & 60 & 55 & 50 & 45 & 40 & \underset{\mathrm{ppm}}{35} & 30 & 25 & 20 & 15 & 10 & 5 & 0 & -5 & -10 & -20 & -30 & \end{array}$

${ }^{1} \mathbf{H} \mathbf{N M R}\left(600 \mathrm{MHz}, \mathrm{CD}_{2} \mathrm{Cl}_{2}\right)$ spectrum of $\mathbf{1 b}$

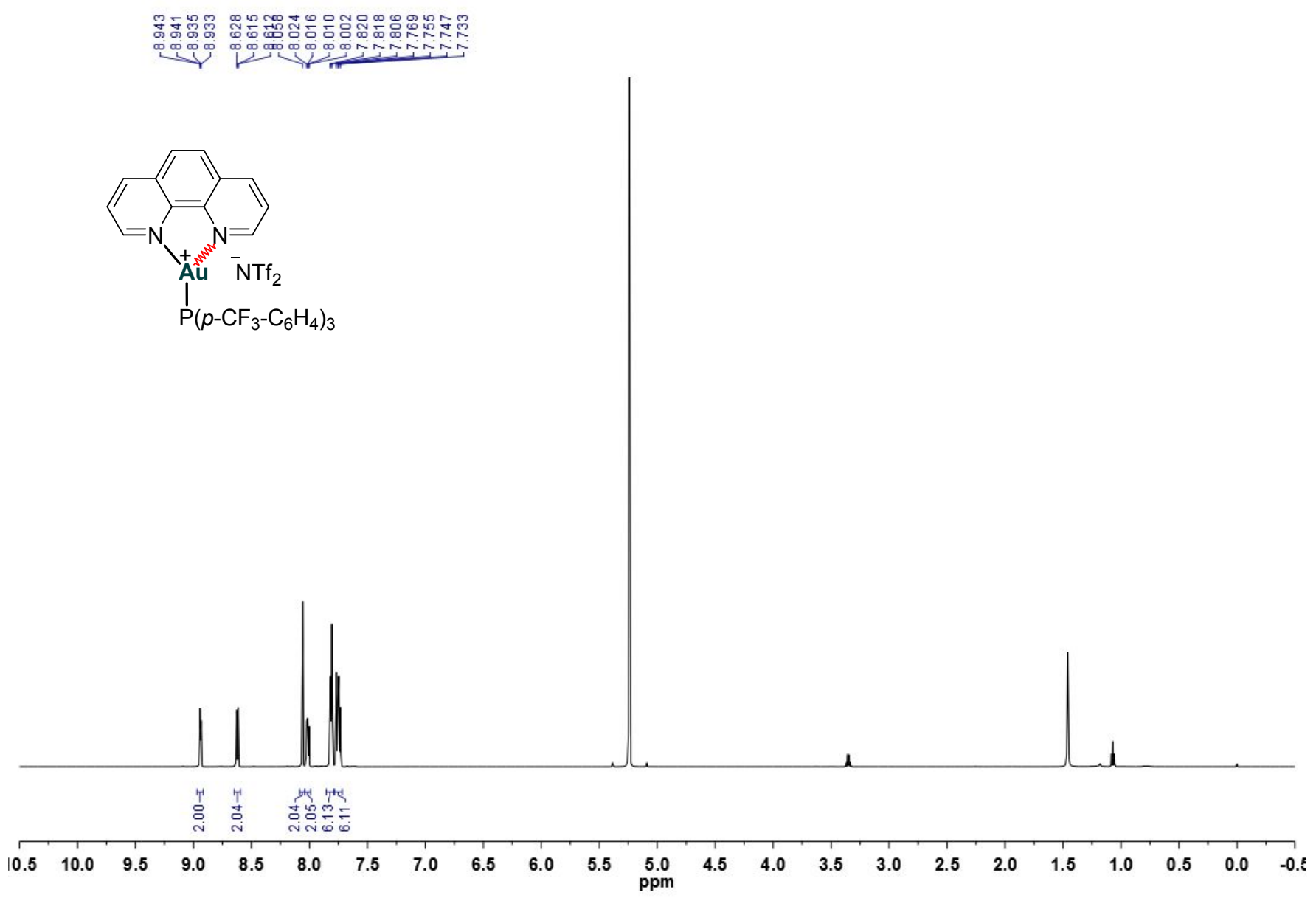


${ }^{13} \mathbf{C ~ N M R}\left(151 \mathrm{MHz}, \mathrm{CD}_{2} \mathrm{Cl}_{2}\right)$ spectrum of $\mathbf{1 b}$

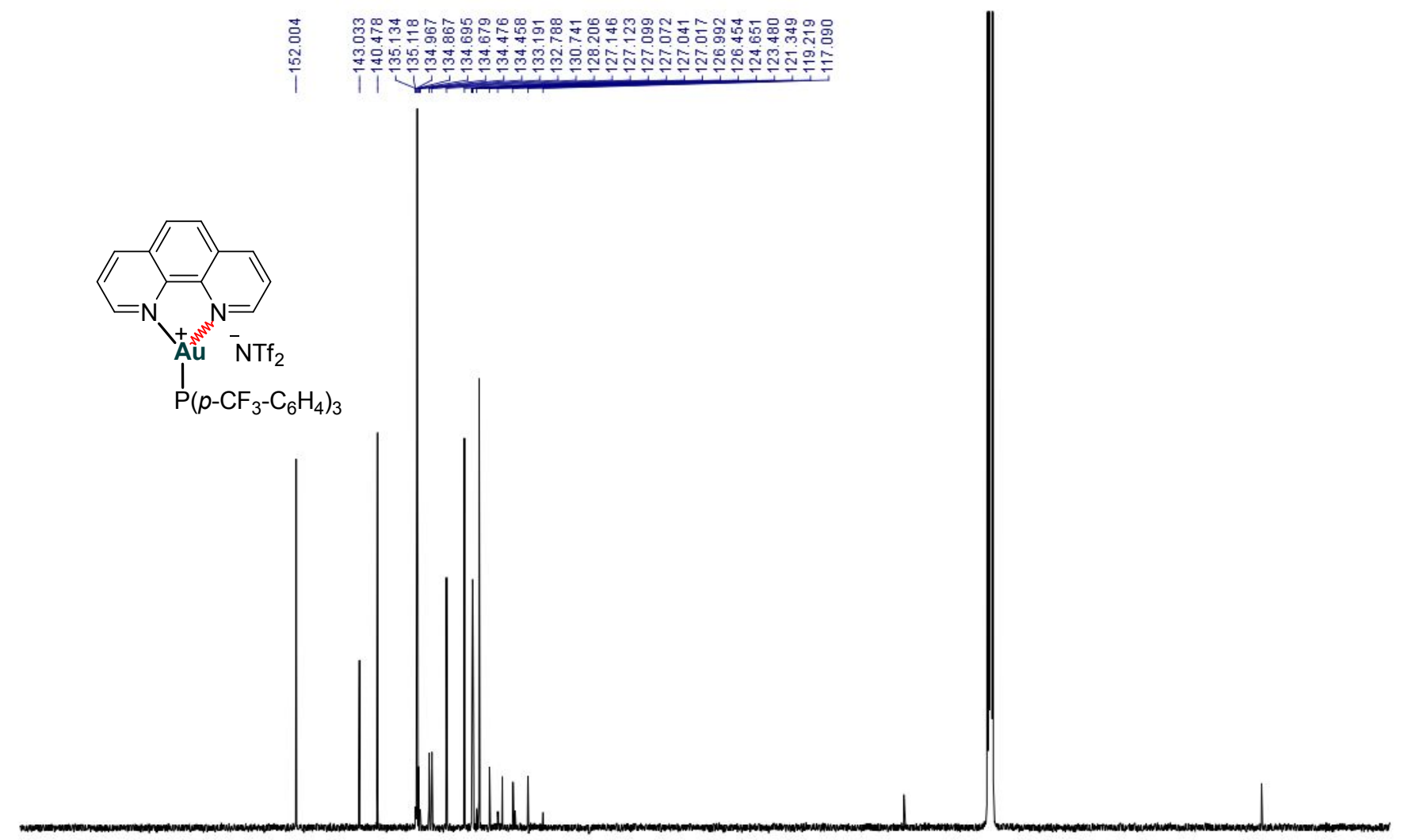

190

$180 \quad 170 \quad 160$

$150 \quad 140$

130

120

110100 ppm $^{90}$

${ }^{31} \mathbf{P}$ NMR (122 MHz, $\left.\mathrm{CD}_{2} \mathrm{Cl}_{2}\right)$ spectrum of $\mathbf{1 b}$ 

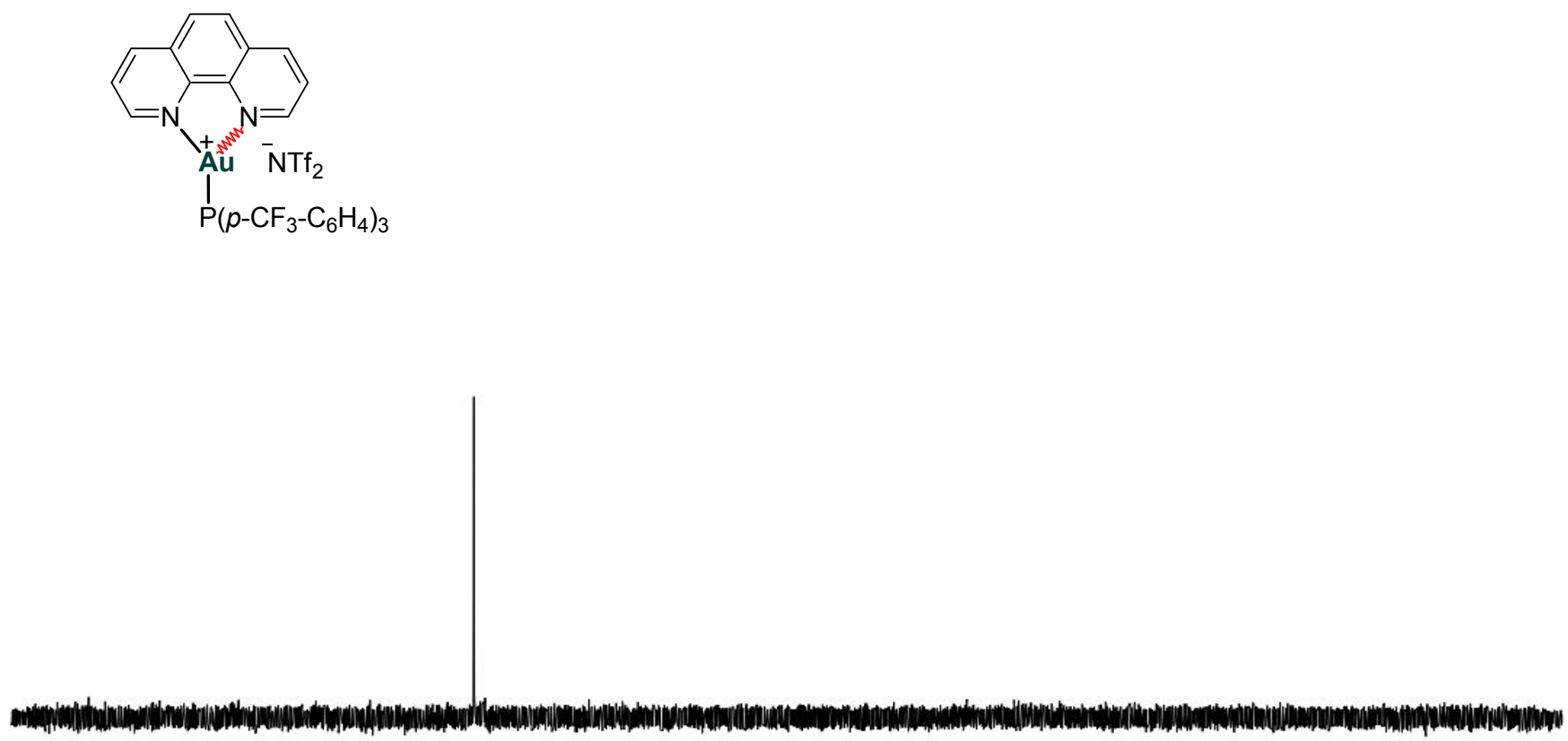

\begin{tabular}{|c|c|c|c|c|c|c|c|c|c|c|c|c|c|}
\hline 1 & 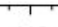 & 1. & 1 & 1 & 1 & 1 & 11 & 11 & 11 & 111 & 11 & 11 & 11 \\
\hline 140 & 110 & 80 & 60 & 40 & 20 & 0 & -20 & $\begin{array}{c}-50 \\
\mathrm{ppm}\end{array}$ & -80 & -110 & -140 & -180 & -220 \\
\hline
\end{tabular}

${ }^{1} \mathbf{H}$ NMR $\left(600 \mathrm{MHz}, \mathrm{CD}_{2} \mathrm{Cl}_{2}\right)$ spectrum of $\mathbf{1 c}$

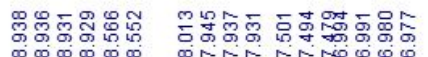

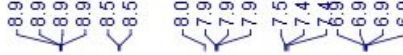
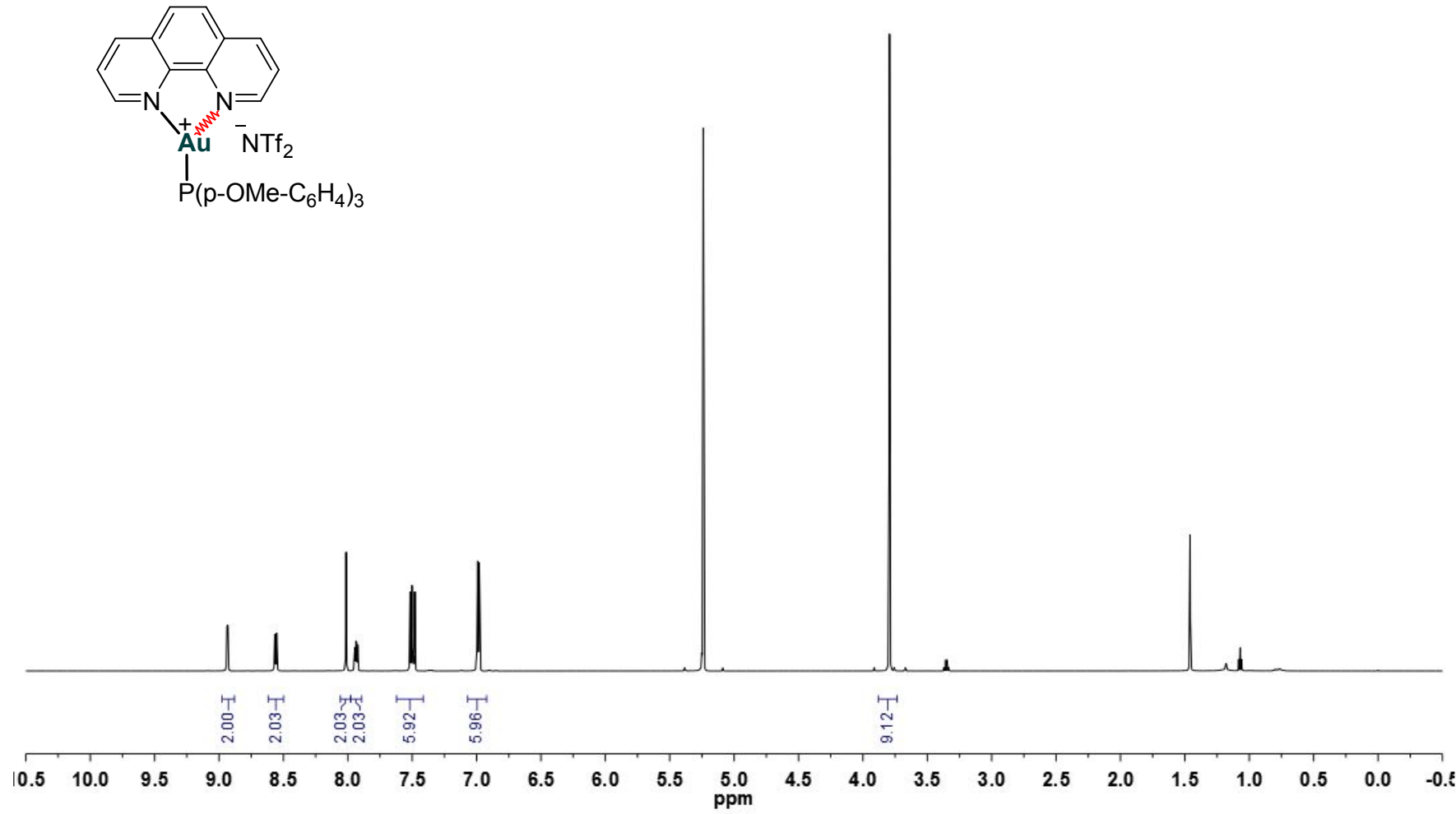
${ }^{13} \mathrm{C}$ NMR (151 MHz, $\left.\mathrm{CD}_{2} \mathrm{Cl}_{2}\right)$ spectrum of 1c

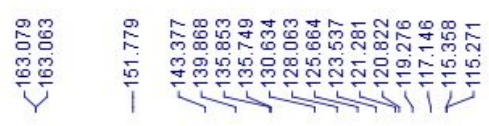
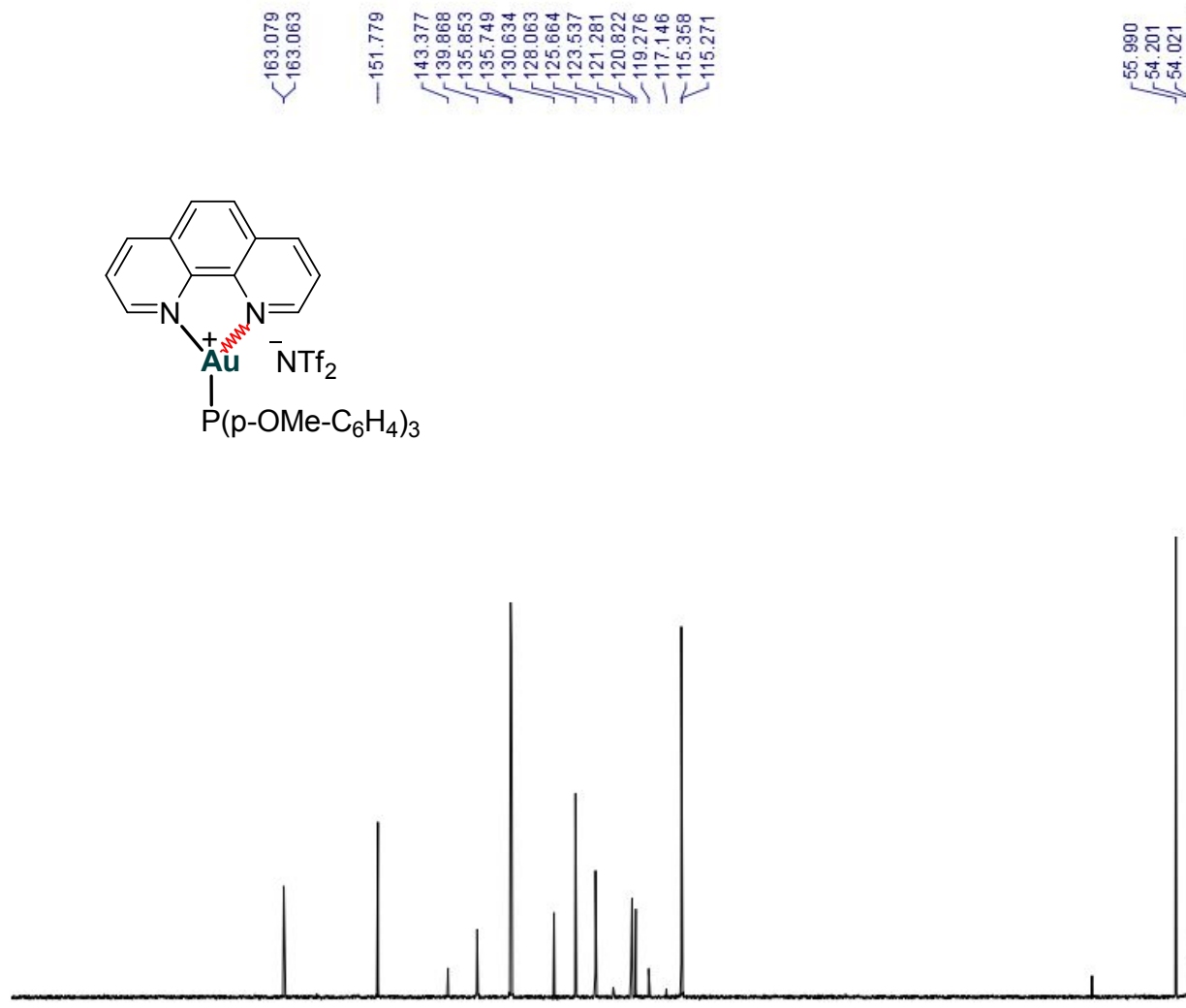

${ }^{31} \mathbf{P}$ NMR (122 MHz, $\left.\mathrm{CD}_{2} \mathrm{Cl}_{2}\right)$ spectrum of 1c 


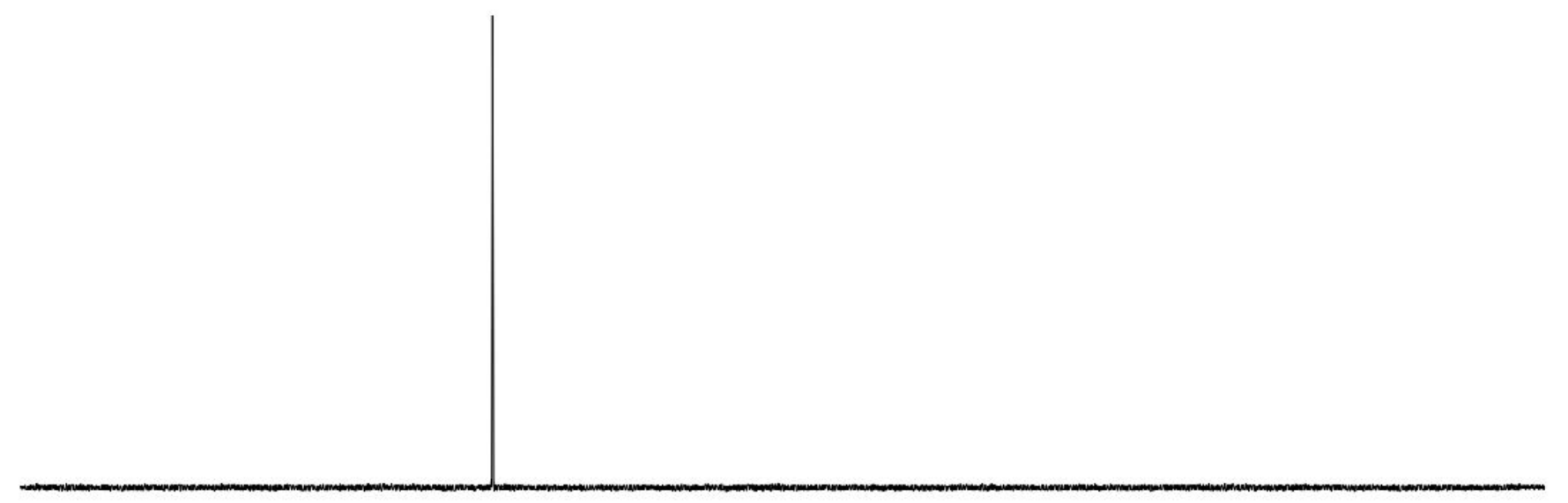

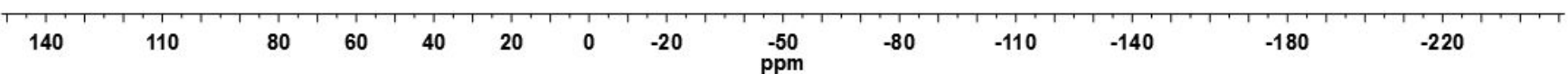

${ }^{1} \mathbf{H}$ NMR (600 MHz, DMSO) spectrum of 1d 


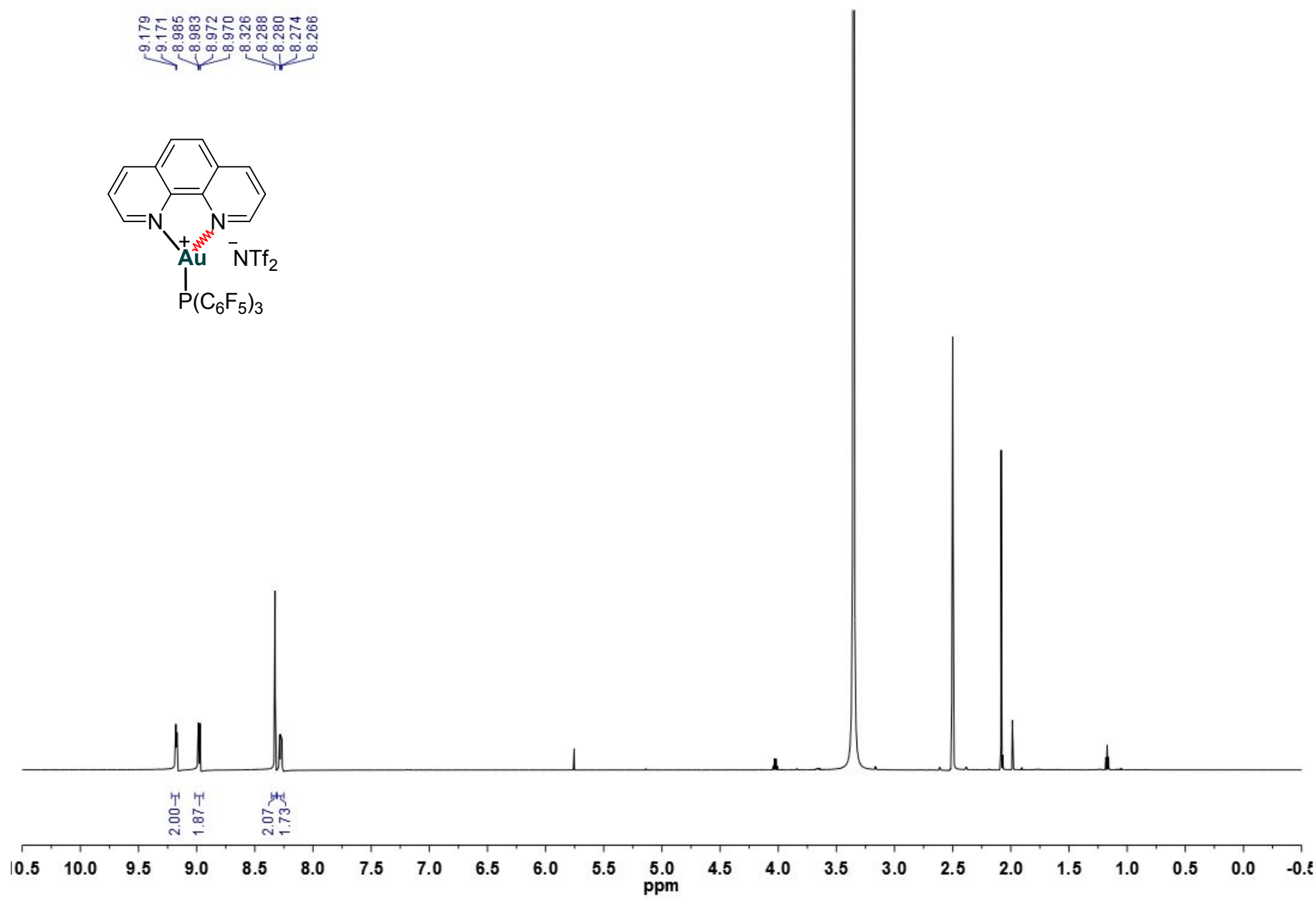

${ }^{\mathbf{1}} \mathbf{H}$ NMR $\left(600 \mathrm{MHz}, \mathrm{CD}_{2} \mathrm{Cl}_{2}\right)$ spectrum of $\mathbf{1 e}$ 


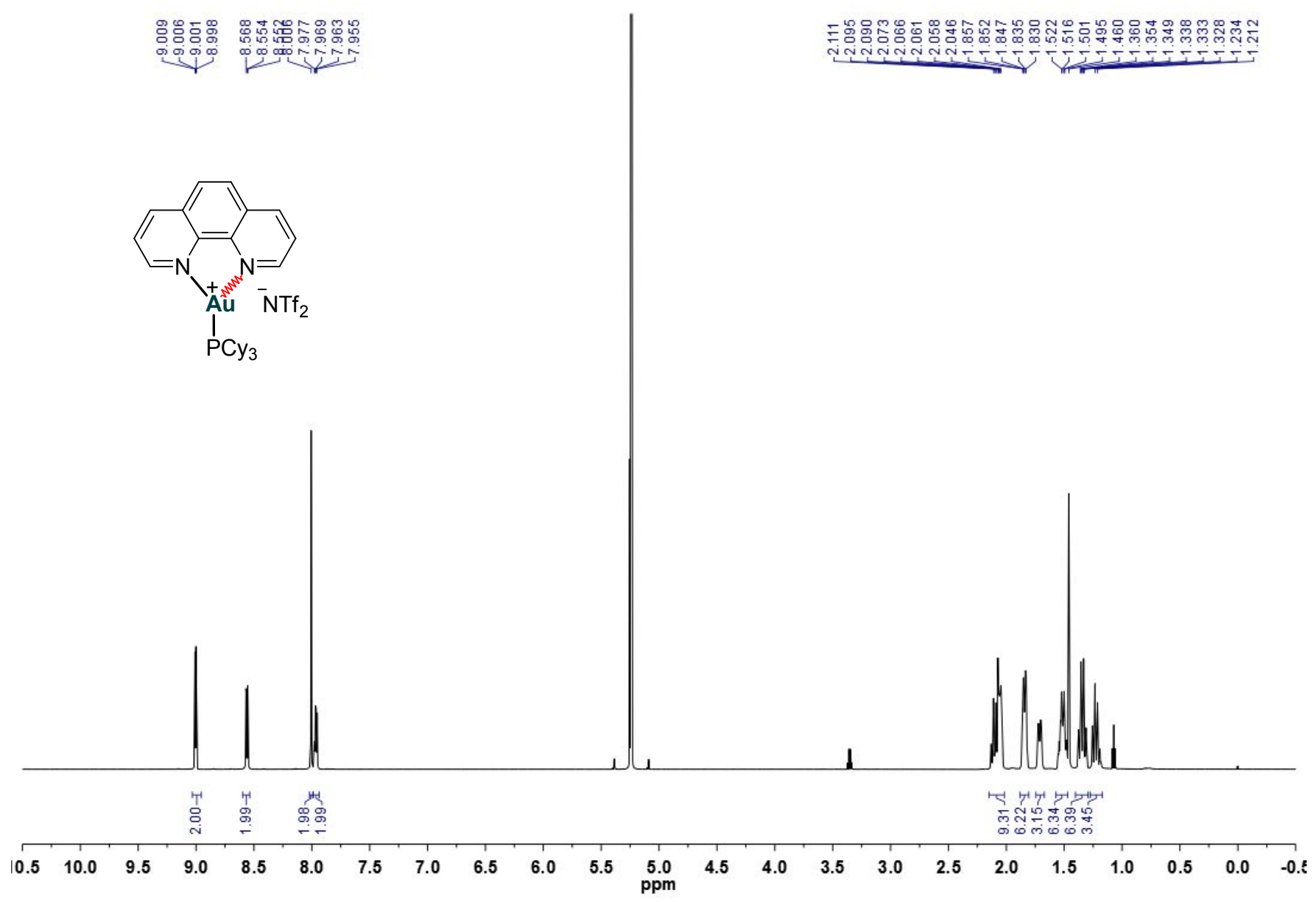

${ }^{13} \mathbf{C}$ NMR $\left(151 \mathrm{MHz}, \mathrm{CD}_{2} \mathrm{Cl}_{2}\right)$ spectrum of $\mathbf{1 e}$

广
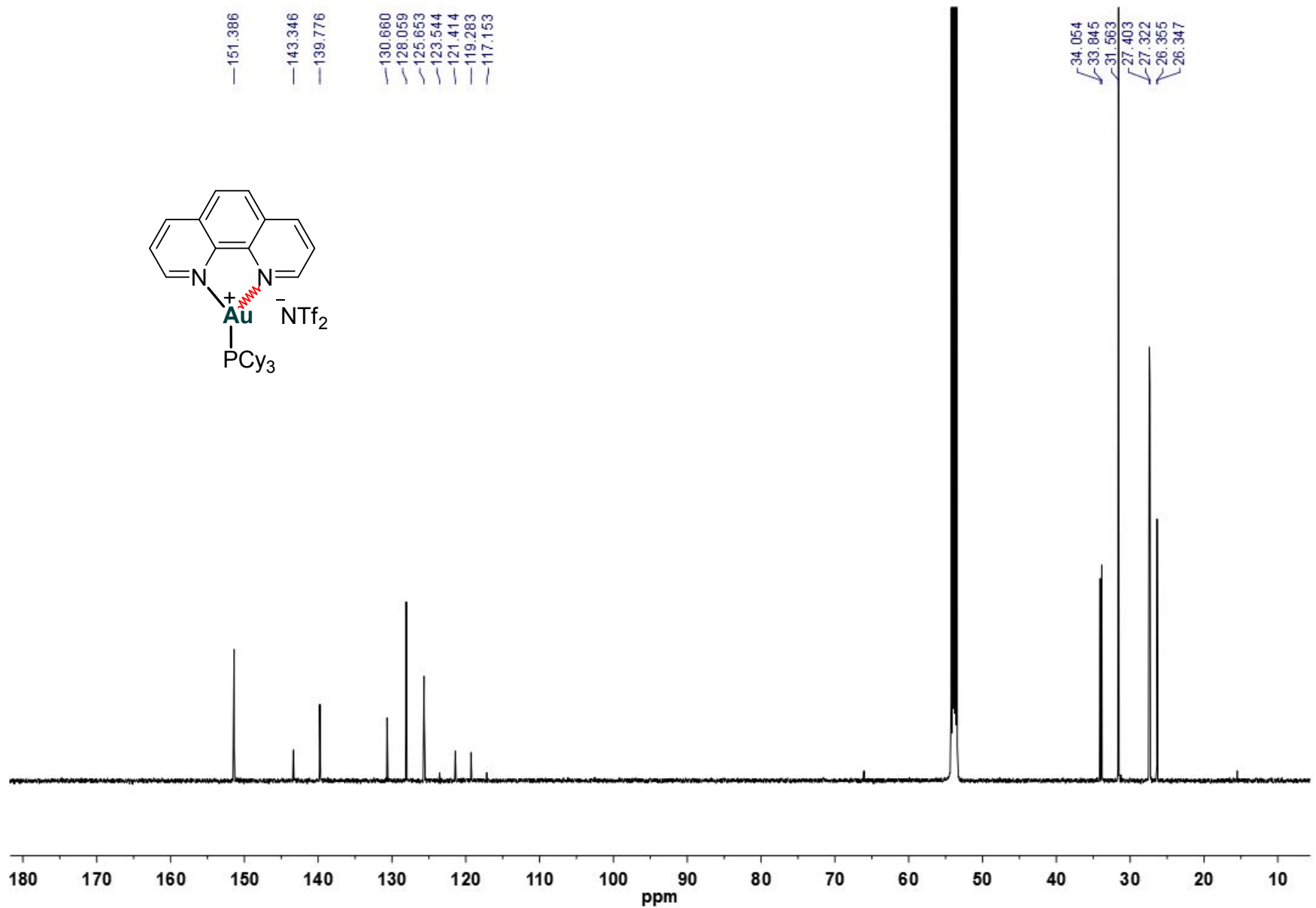
${ }^{31} \mathbf{P}$ NMR (122 MHz, $\left.\mathrm{CD}_{3} \mathrm{CN}\right)$ spectrum of $1 \mathbf{e}$

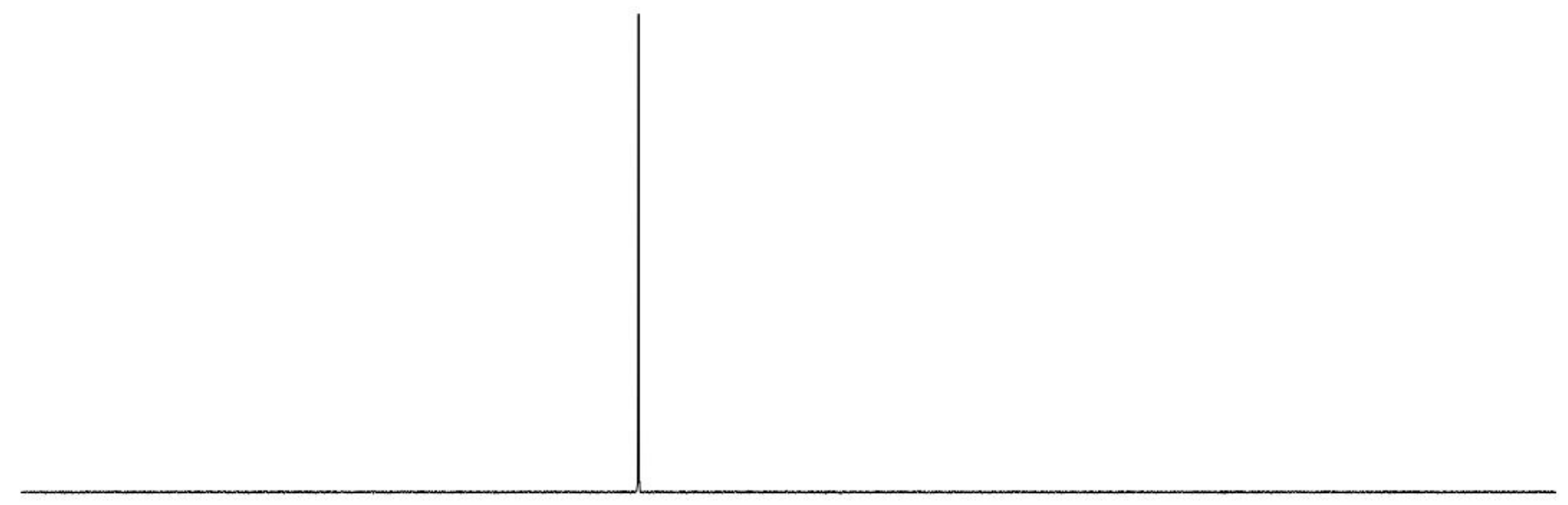

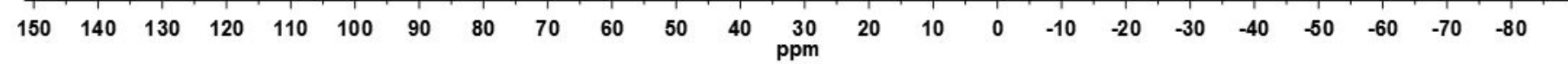

${ }^{\mathbf{1}} \mathbf{H}$ NMR $\left(600 \mathrm{MHz}, \mathrm{CD}_{2} \mathrm{Cl}_{2}\right)$ spectrum of $\mathbf{1 f}$ 

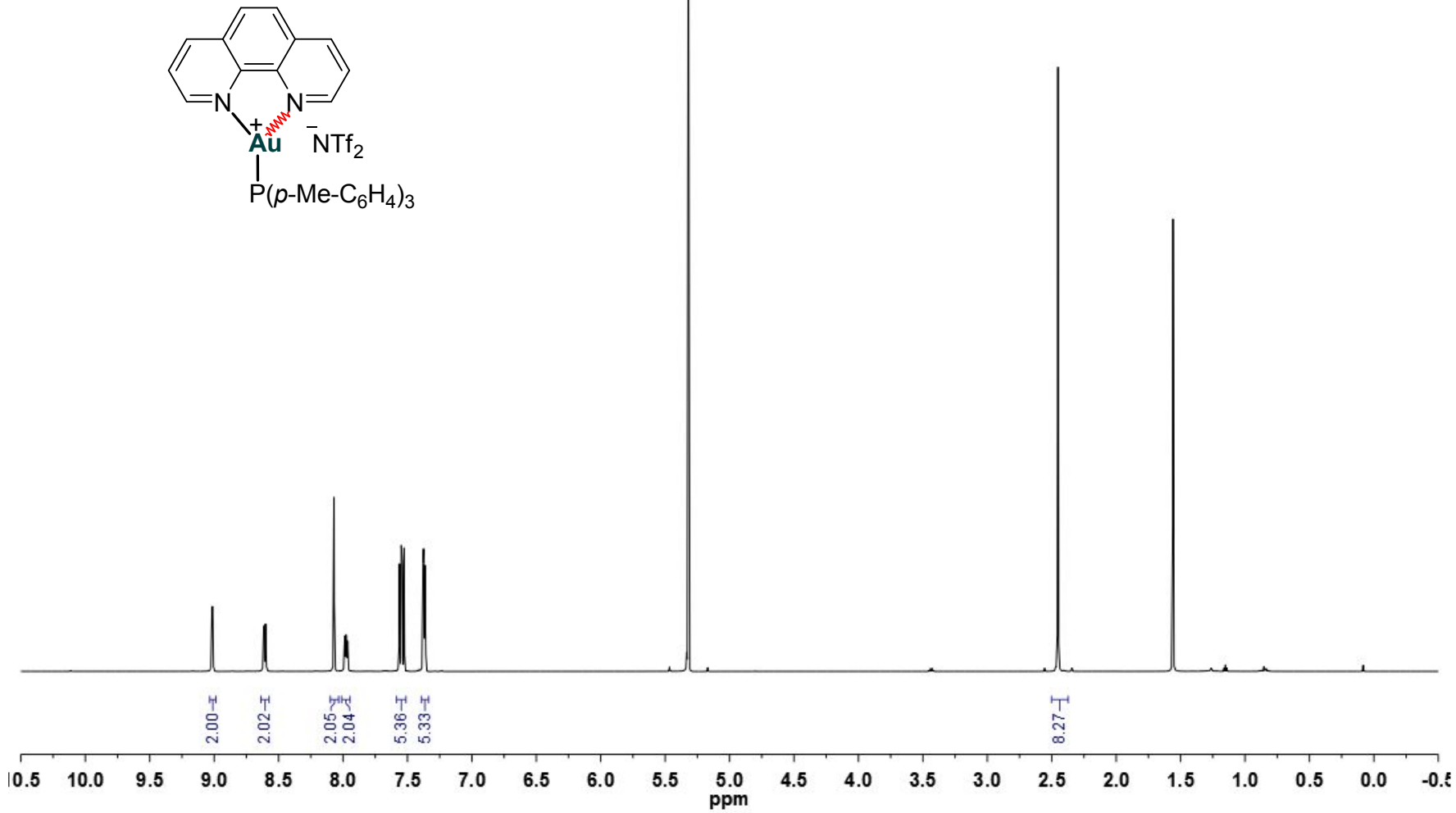

${ }^{13} \mathbf{C}$ NMR $\left(151 \mathrm{MHz}, \mathrm{CD}_{2} \mathrm{Cl}_{2}\right)$ spectrum of $\mathbf{1 f}$
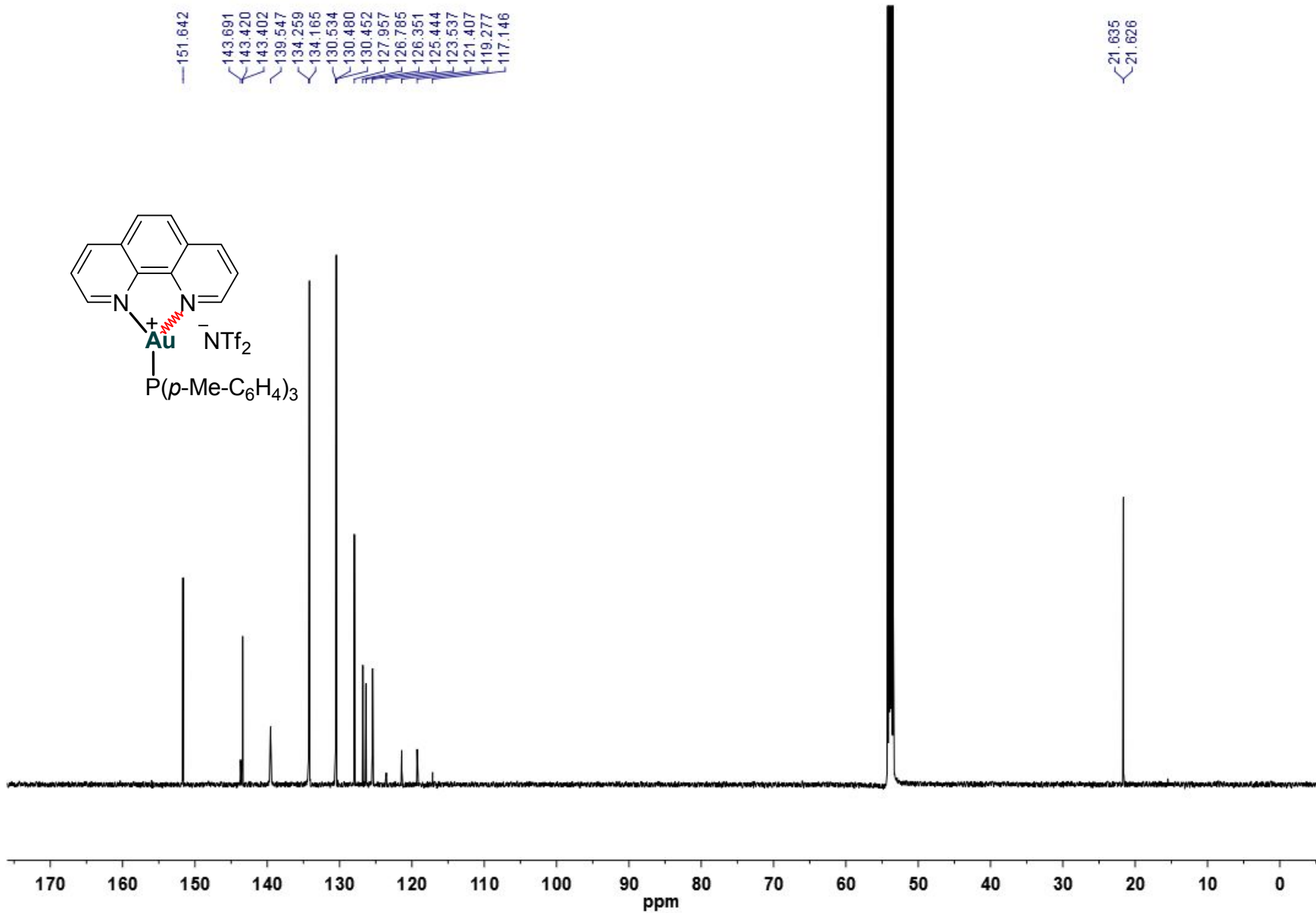
${ }^{31} \mathbf{P}$ NMR (122 MHz, $\left.\mathrm{CD}_{2} \mathrm{Cl}_{2}\right)$ spectrum of $\mathbf{1 f}$

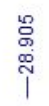

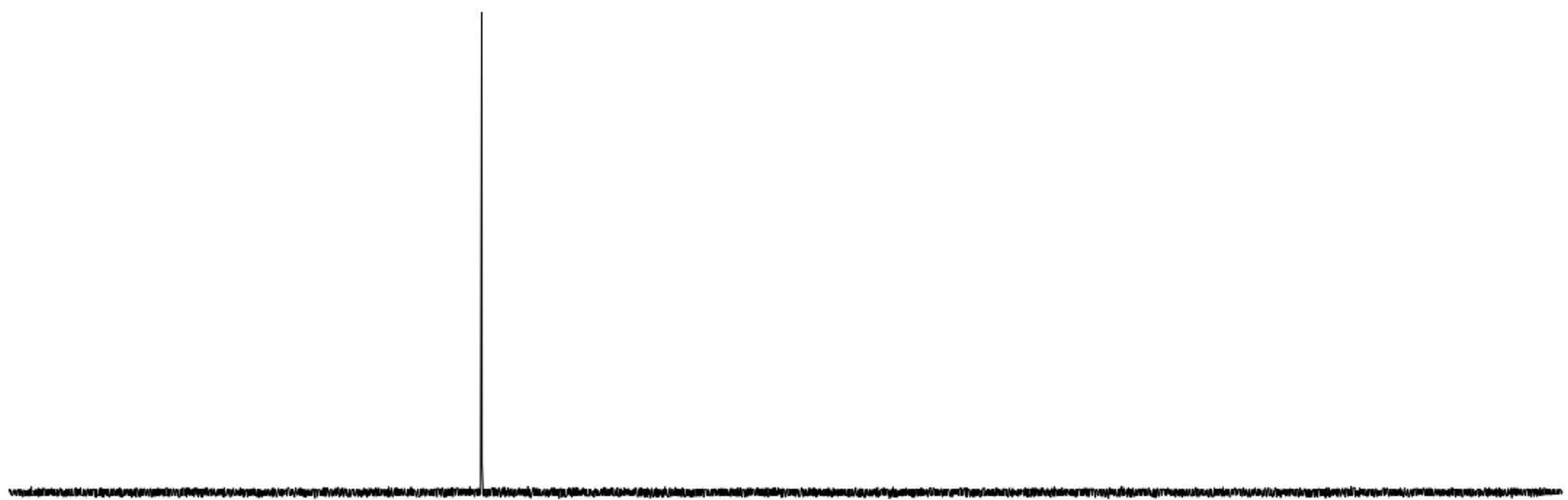

\begin{tabular}{llllllllllllll}
\hline 140 & 110 & 80 & 60 & 40 & 20 & 0 & -20 & $\begin{array}{c}-50 \\
\mathrm{ppm}\end{array}$ & -80 & -110 & -140 & -180 & -220 \\
\end{tabular}

${ }^{1} \mathbf{H}$ NMR $\left(400 \mathrm{MHz}, \mathrm{CD}_{2} \mathrm{Cl}_{2}\right)$ spectrum of $\mathbf{1 g}$ 

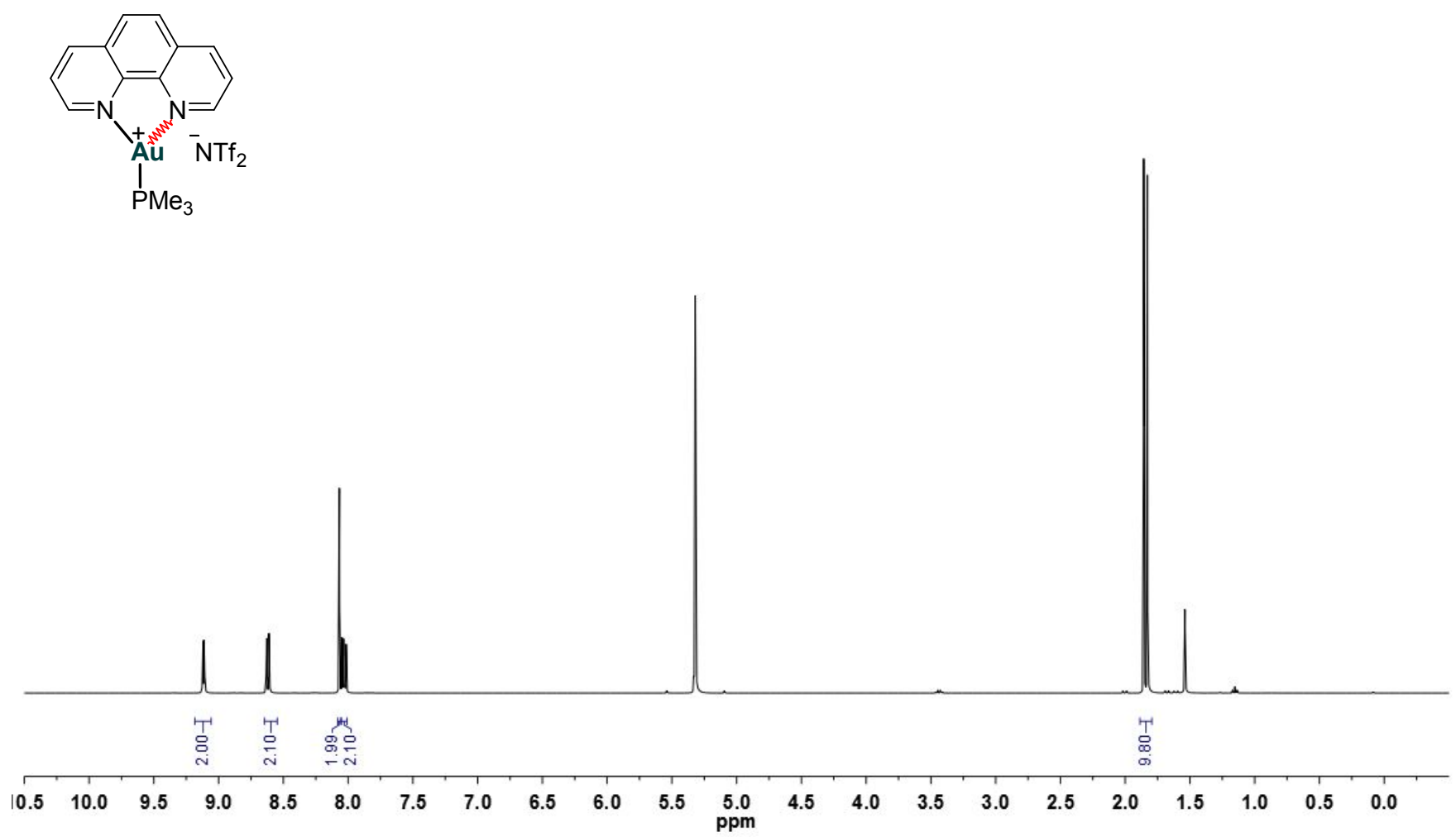

${ }^{13} \mathbf{C ~ N M R}\left(101 \mathrm{MHz}, \mathrm{CD}_{2} \mathrm{Cl}_{2}\right)$ spectrum of $\mathbf{1 g}$ 

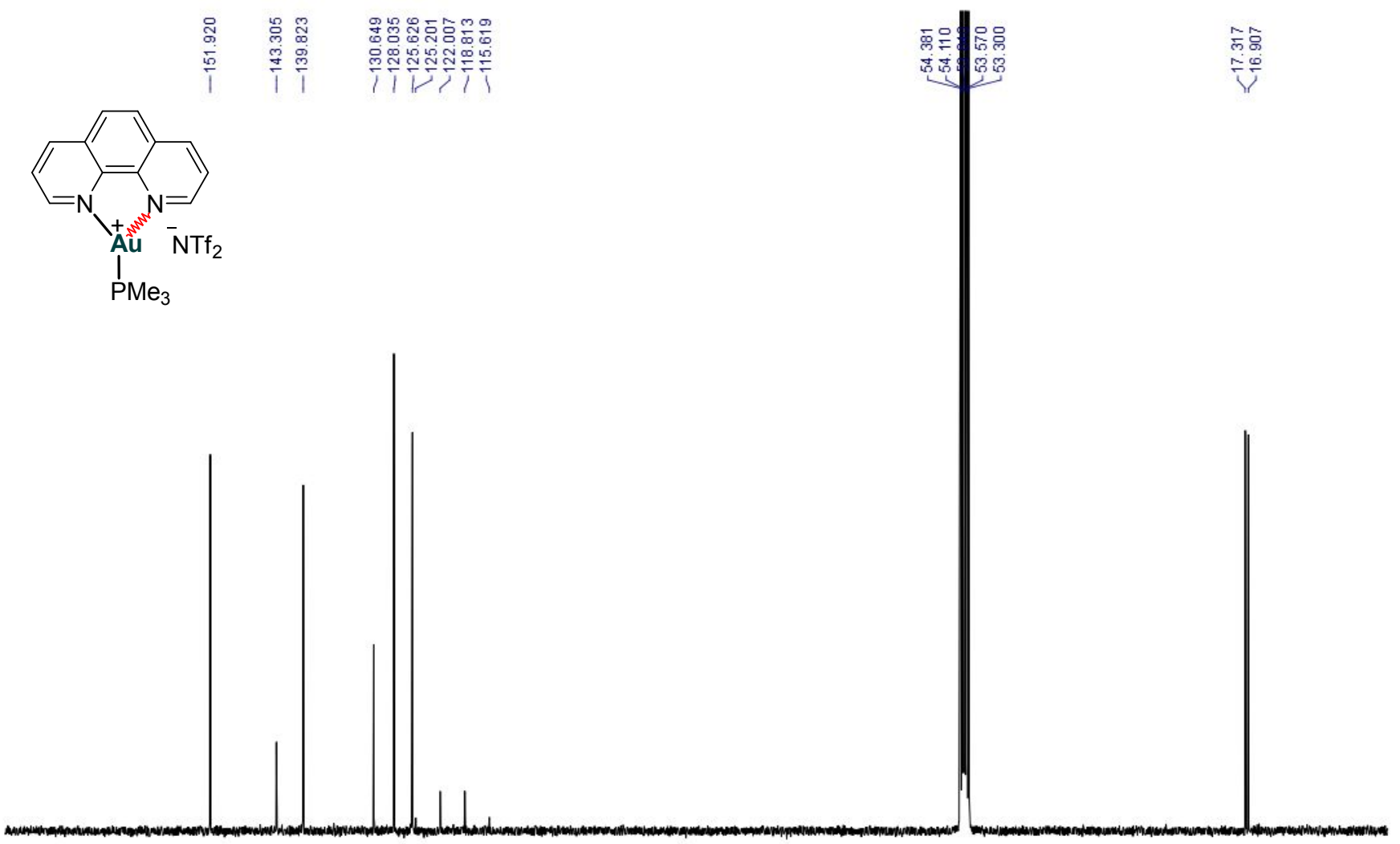

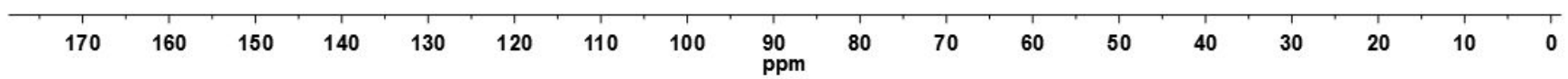

${ }^{31} \mathbf{P}$ NMR $\left(122 \mathrm{MHz}, \mathrm{CD}_{2} \mathrm{Cl}_{2}\right)$ spectrum of $\mathbf{1 g}$

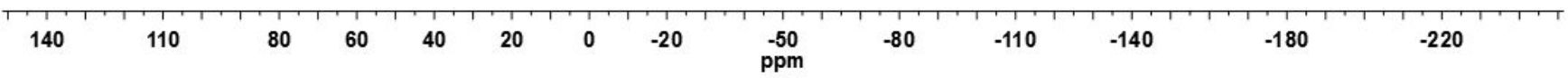


${ }^{1} \mathbf{H}$ NMR $\left(301 \mathrm{MHz}, \mathrm{CD}_{2} \mathrm{Cl}_{2}\right)$ spectrum of $\mathbf{6 a}$

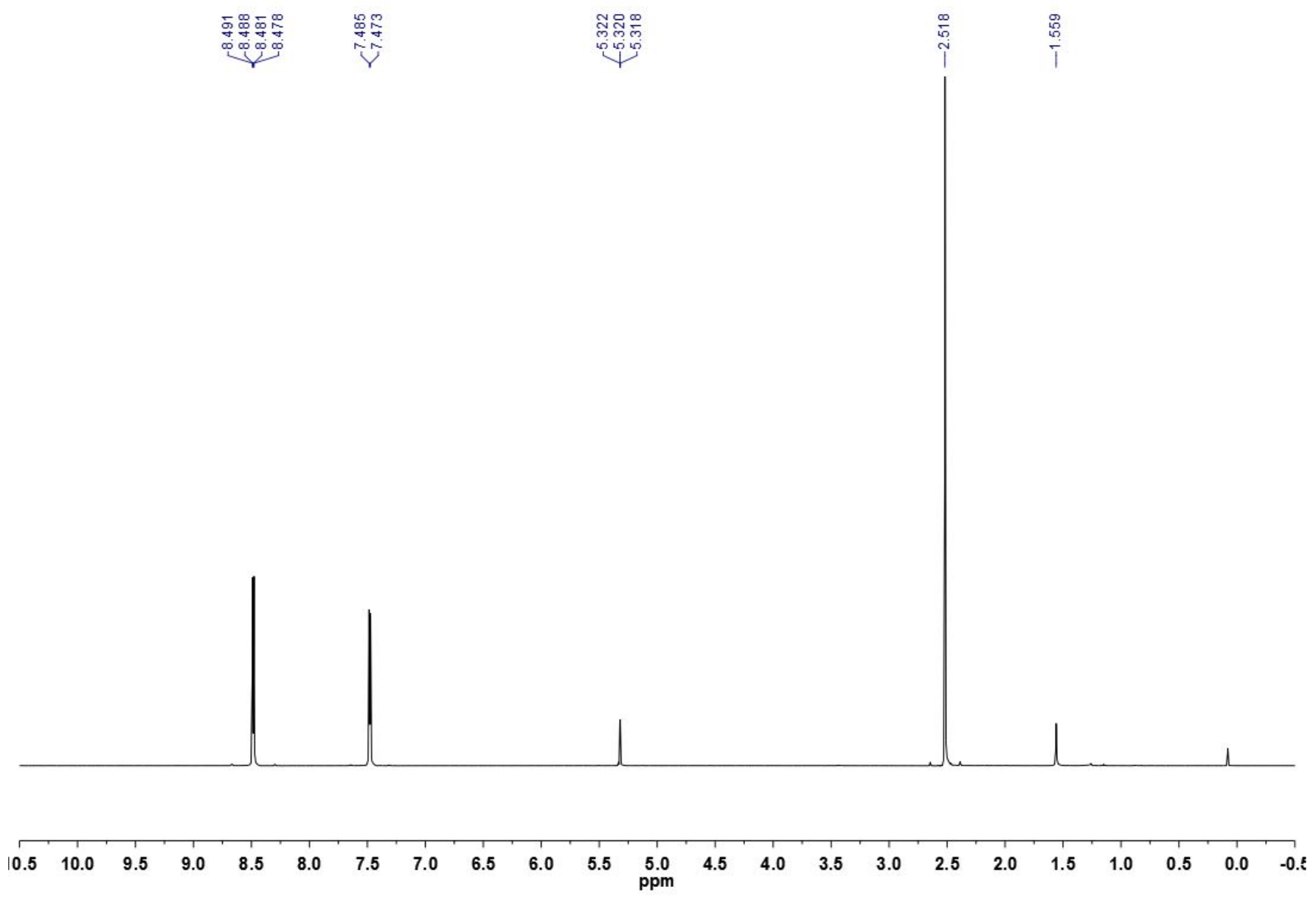

${ }^{13} \mathbf{C}$ NMR (126 MHz, $\left.\mathrm{CD}_{2} \mathrm{Cl}_{2}\right)$ spectrum of $\mathbf{6 a}$ 

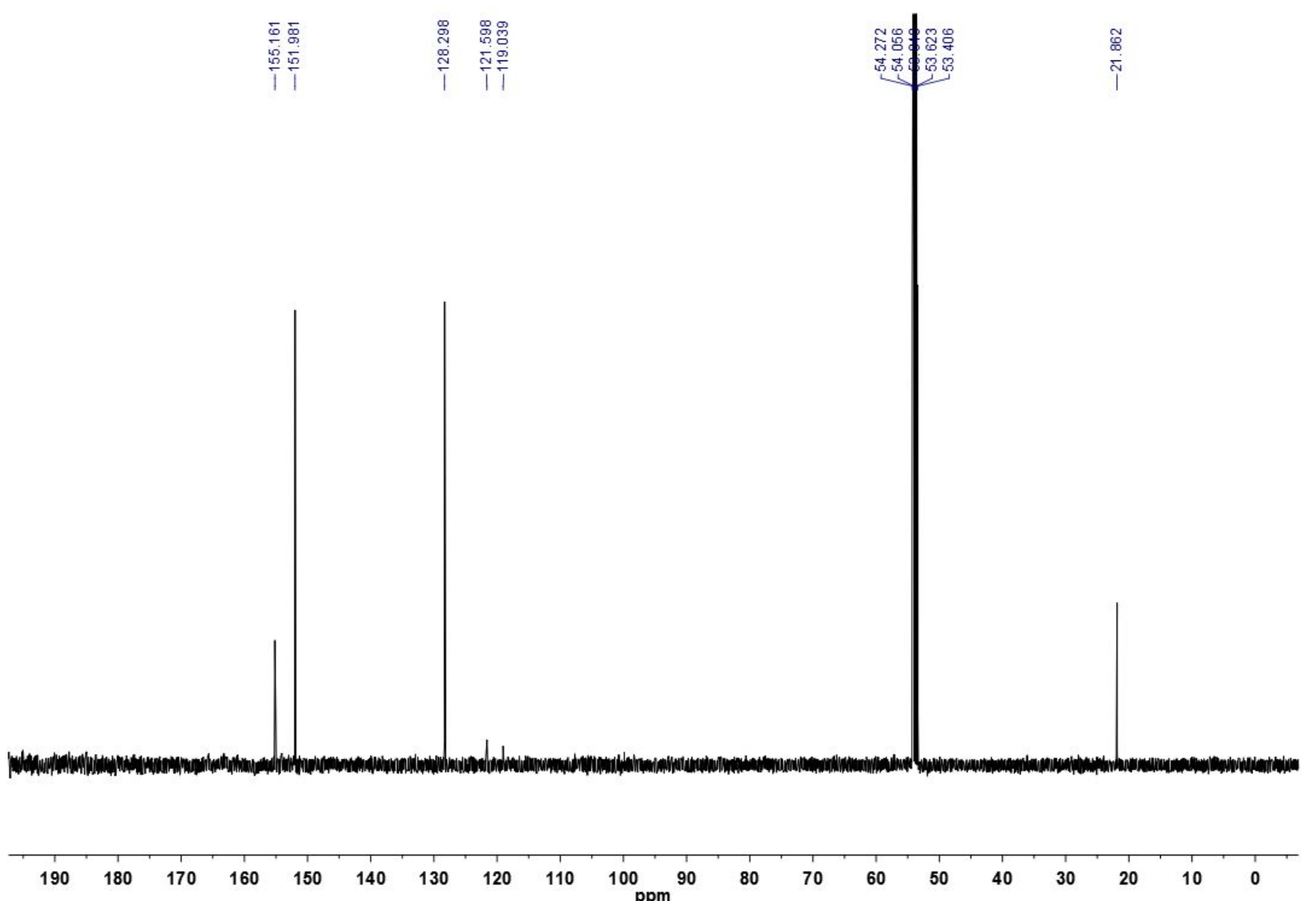

${ }^{19}$ F NMR $\left(471 \mathrm{MHz}, \mathrm{CD}_{2} \mathrm{Cl}_{2}\right)$ spectrum of $\mathbf{6 a}$

\begin{tabular}{lllllllllllllllllll}
\hline 40 & 30 & 20 & 10 & 0 & -10 & -30 & -50 & -70 & -90 & -110 & -130 & -150 & -170 & -190 & -210 & -230 &
\end{tabular}


${ }^{1} \mathbf{H}$ NMR $\left(500 \mathrm{MHz}, \mathrm{CD}_{2} \mathrm{Cl}_{2}\right)$ spectrum of $\mathbf{6 b}$

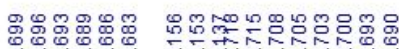

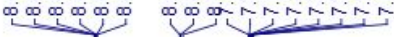

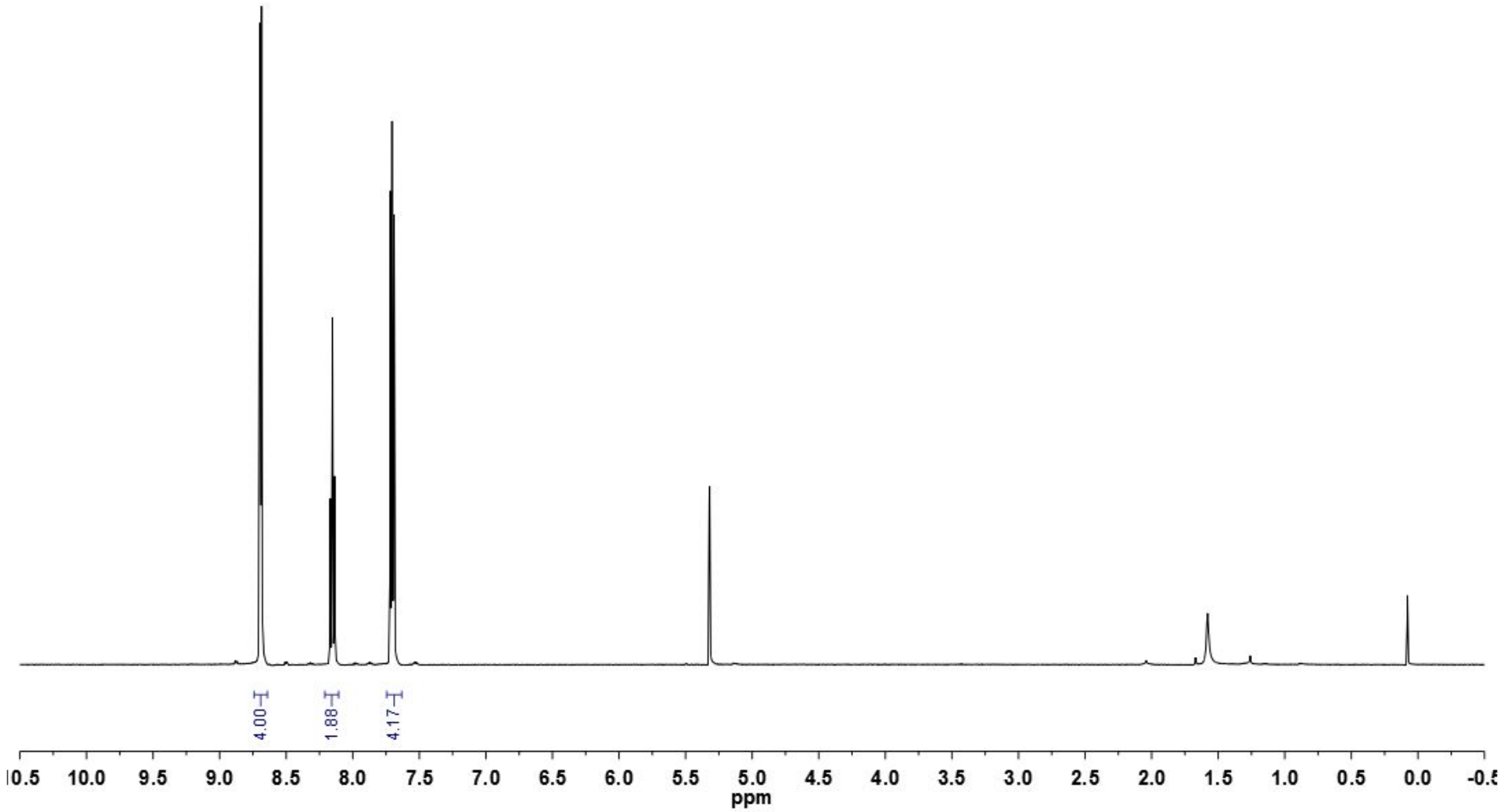

${ }^{13} \mathbf{C}$ NMR $\left(126 \mathrm{MHz}, \mathrm{CD}_{2} \mathrm{Cl}_{2}\right)$ spectrum of $\mathbf{6 b}$ 


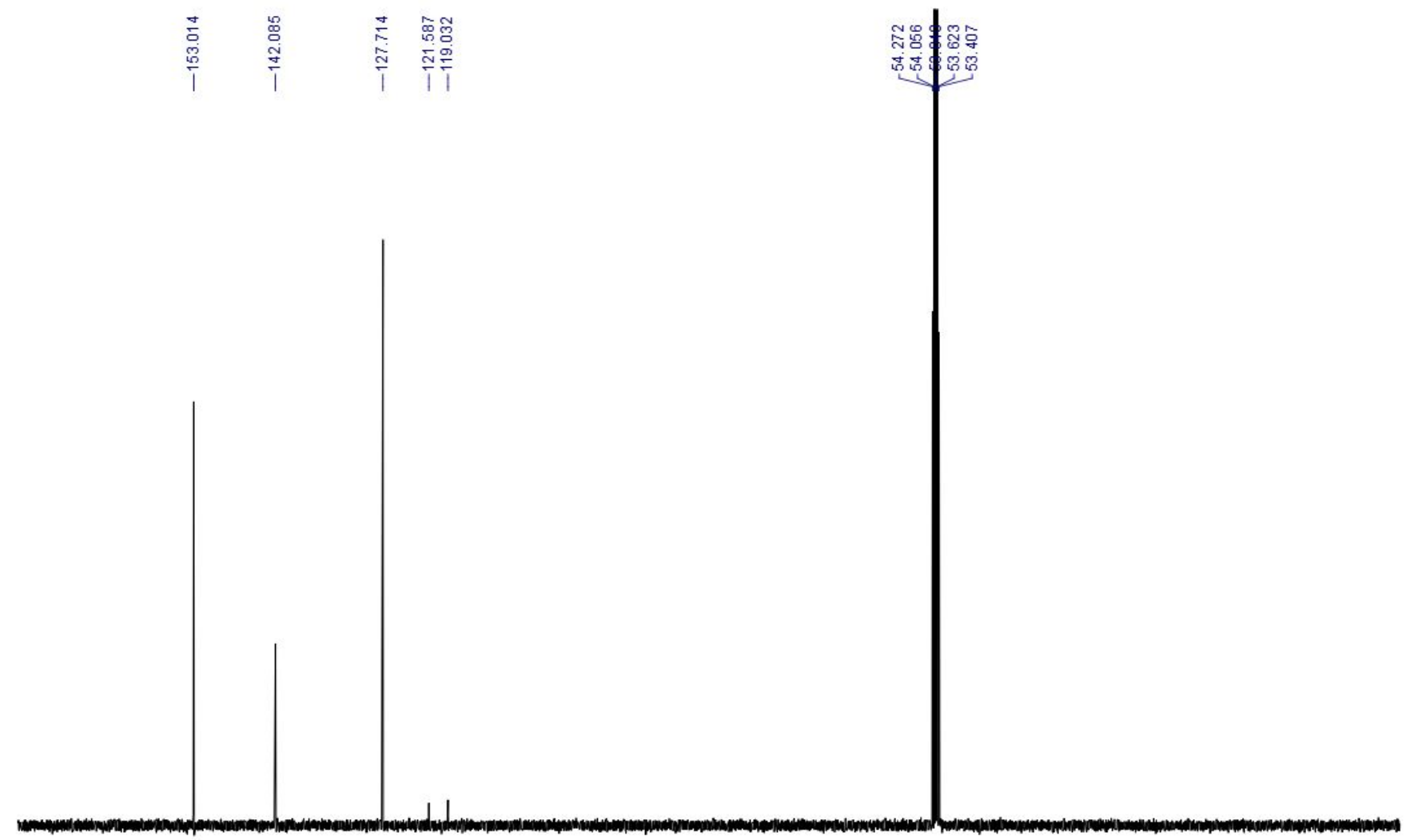

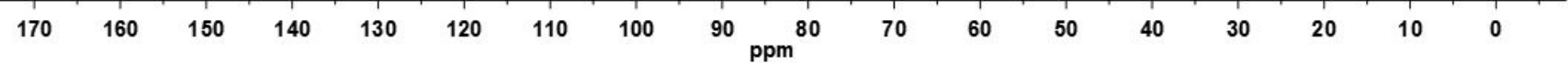

${ }^{19} \mathbf{F}$ NMR (471 MHz, $\mathrm{CD}_{2} \mathrm{Cl}_{2}$ ) spectrum of $\mathbf{6 b}$ 


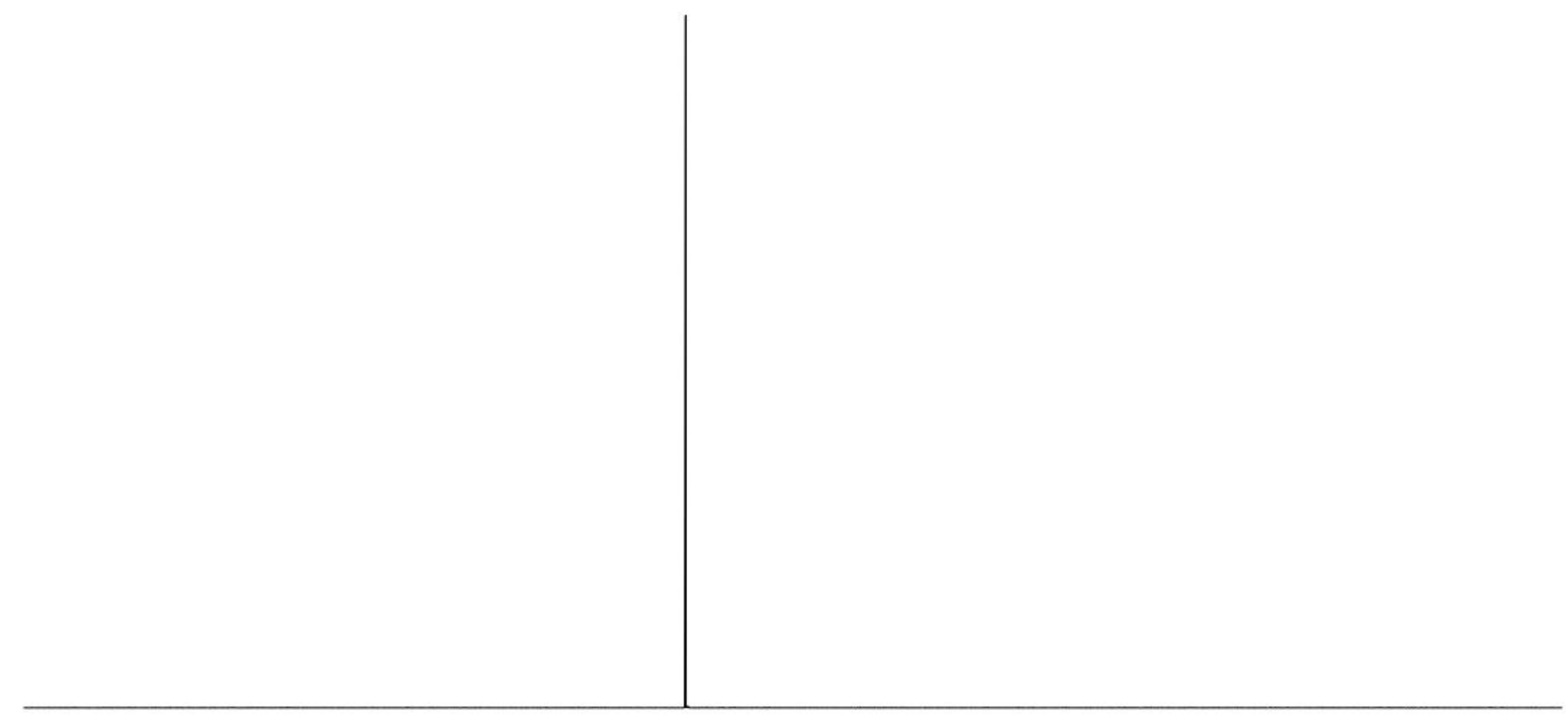

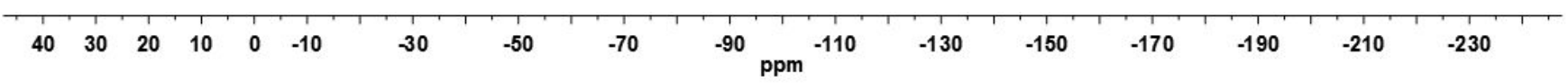

${ }^{1} \mathbf{H}$ NMR $\left(500 \mathrm{MHz}, \mathrm{CD}_{2} \mathrm{Cl}_{2}\right)$ spectrum of $\mathbf{6 c}$

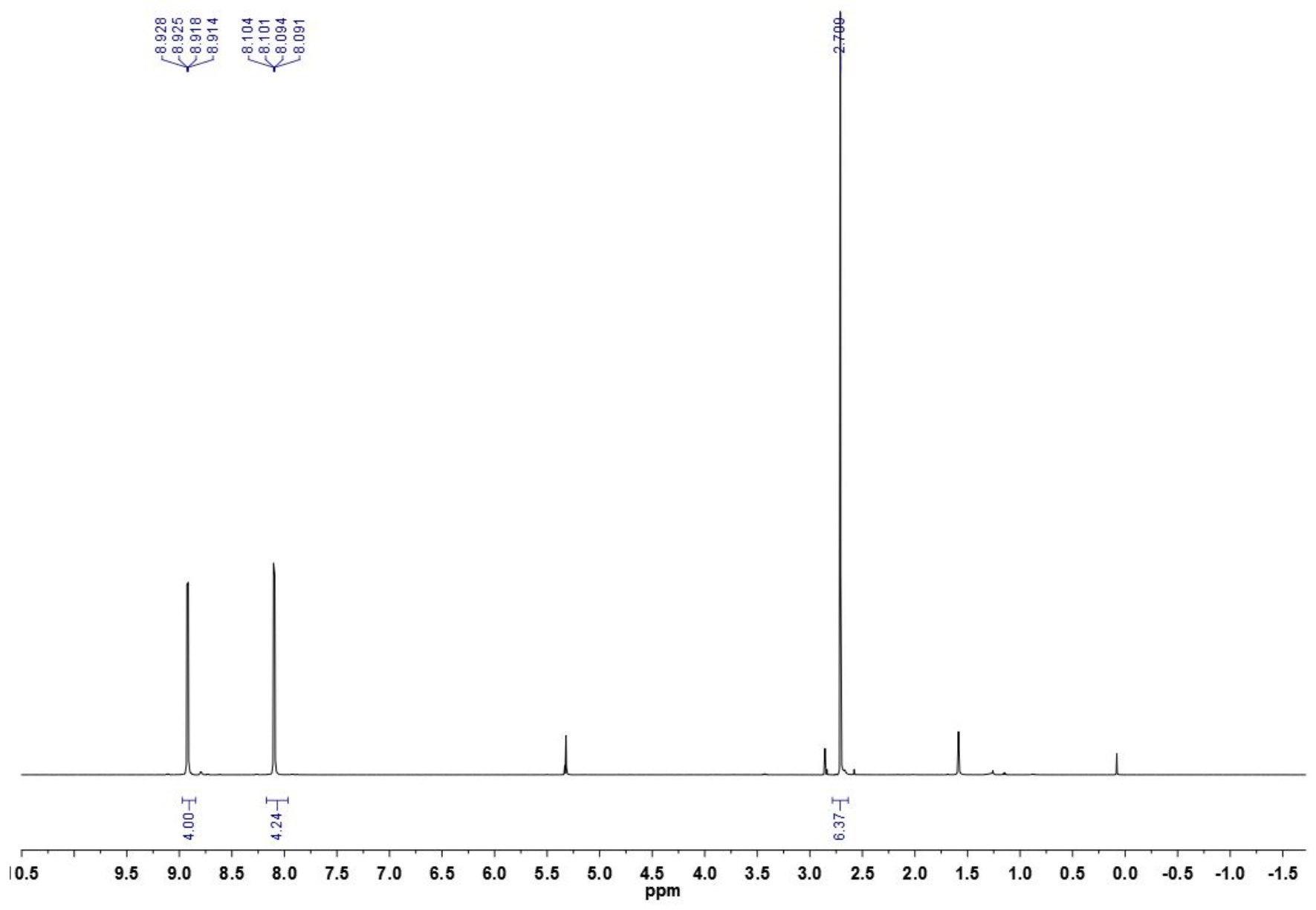


${ }^{13} \mathbf{C}$ NMR (126 MHz, $\left.\mathrm{CD}_{2} \mathrm{Cl}_{2}\right)$ spectrum of $\mathbf{6 c}$

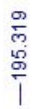

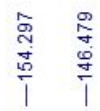

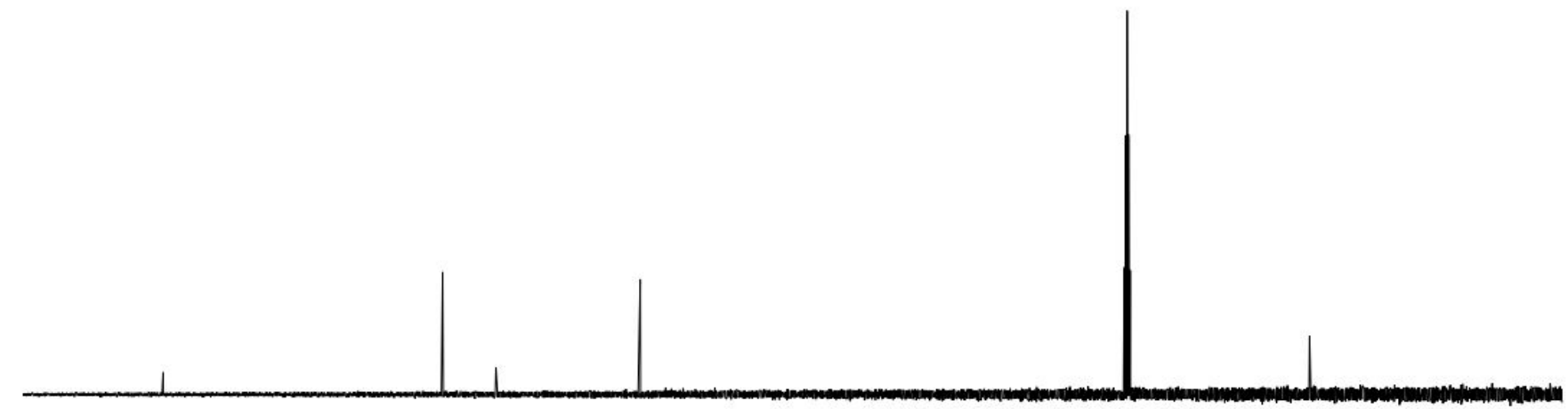

$\begin{array}{llllllllllllllllllllll}210 & 200 & 190 & 180 & 170 & 160 & 150 & 140 & 130 & 120 & 110 \begin{array}{c}100 \\ \mathrm{ppm}\end{array} & 90 & 80 & 70 & 60 & 50 & 40 & 30 & 20 & 10 & 0 & \end{array}$

${ }^{19} \mathbf{F}$ NMR (471 MHz, $\left.\mathrm{CD}_{2} \mathrm{Cl}_{2}\right)$ spectrum of $6 \mathbf{c}$ 


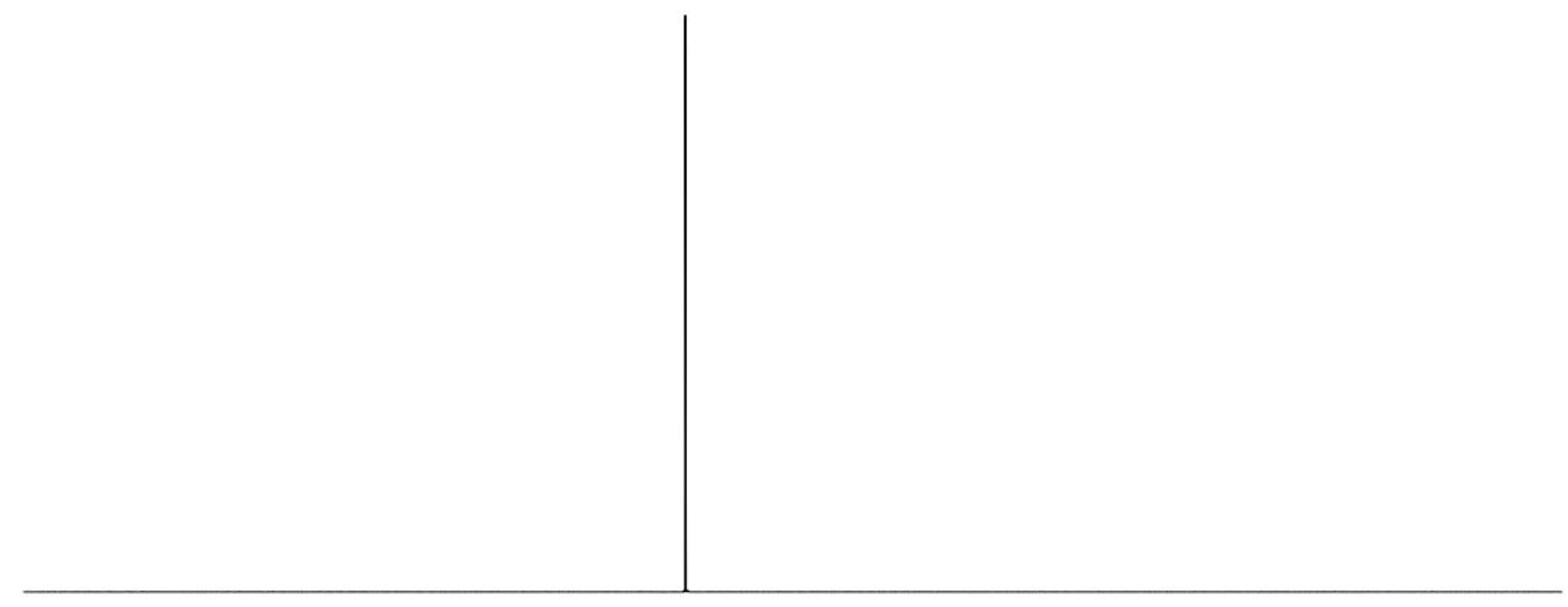

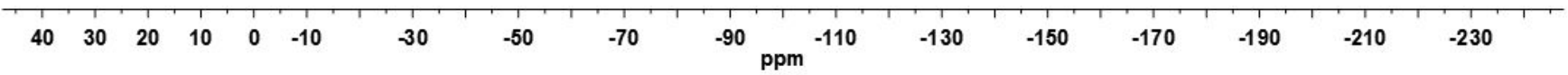

${ }^{1} \mathbf{H}$ NMR (500 MHz, $\mathrm{CD}_{2} \mathrm{Cl}_{2}$ ) spectrum of $\mathbf{6 d}$ 


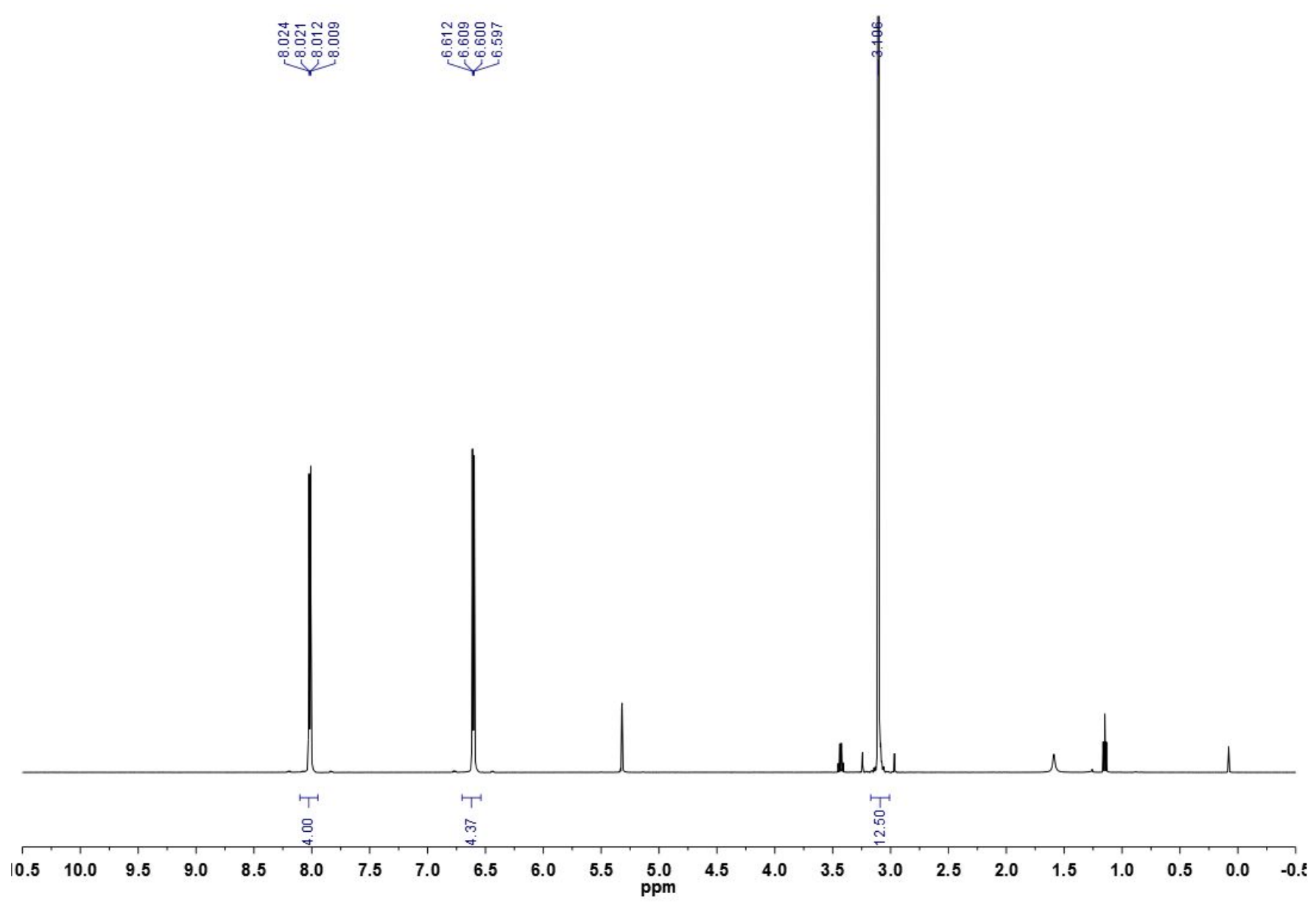

${ }^{13} \mathbf{C}$ NMR (126 MHz, $\mathrm{CD}_{2} \mathrm{Cl}_{2}$ ) spectrum of $\mathbf{6 d}$
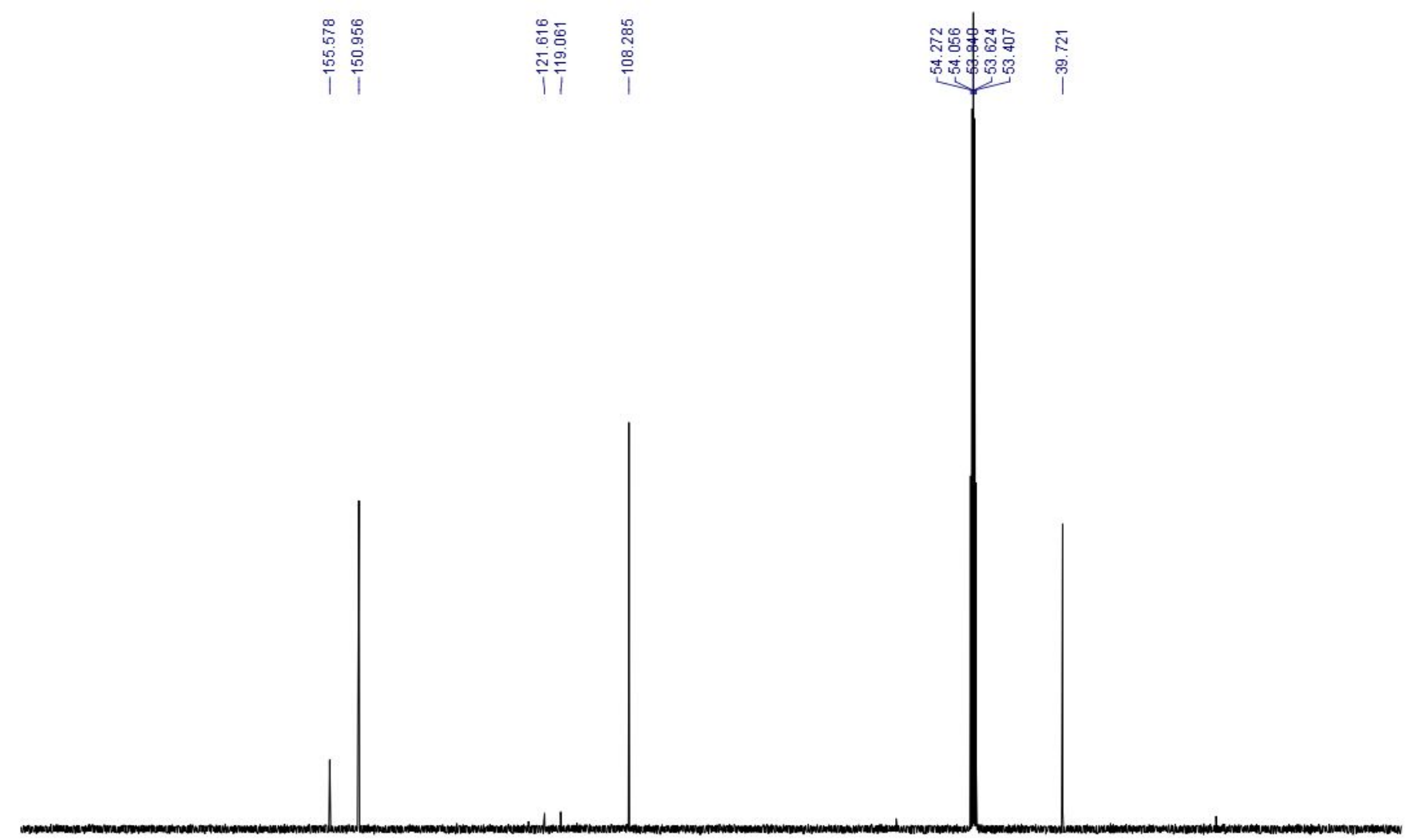
${ }^{19} \mathbf{F}$ NMR (471 MHz, $\left.\mathrm{CD}_{2} \mathrm{Cl}_{2}\right)$ spectrum of $\mathbf{6 d}$

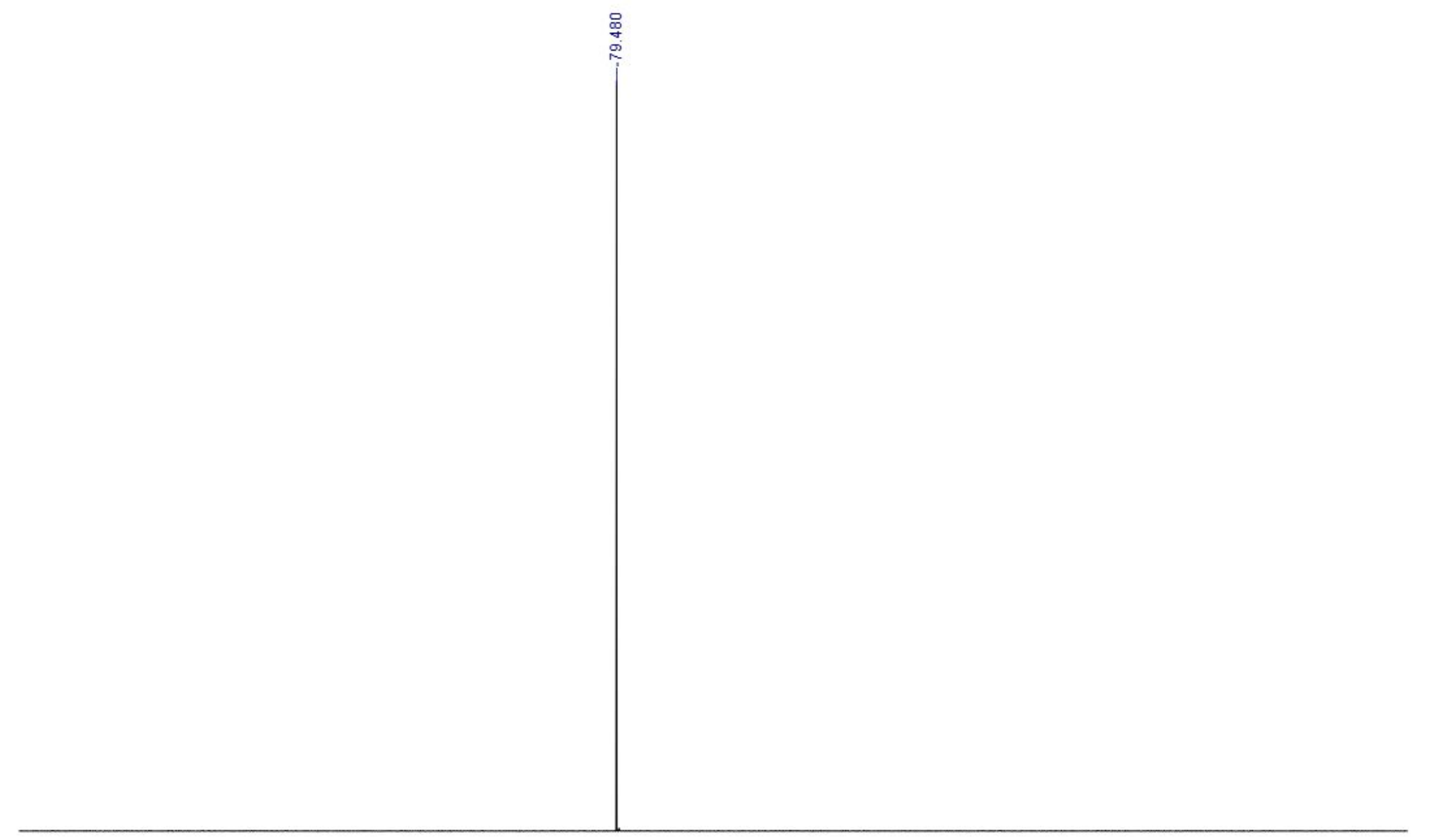

\begin{tabular}{llllllllllllllllll}
\hline 40 & 30 & 20 & 10 & 0 & -10 & -30 & -50 & -70 & -90 & -110 & -130 & -150 & -170 & -190 & -210 & -230 &
\end{tabular}

${ }^{1} \mathbf{H}$ NMR (301 MHz, $\mathrm{CD}_{2} \mathrm{Cl}_{2}$ ) spectrum of 7a 


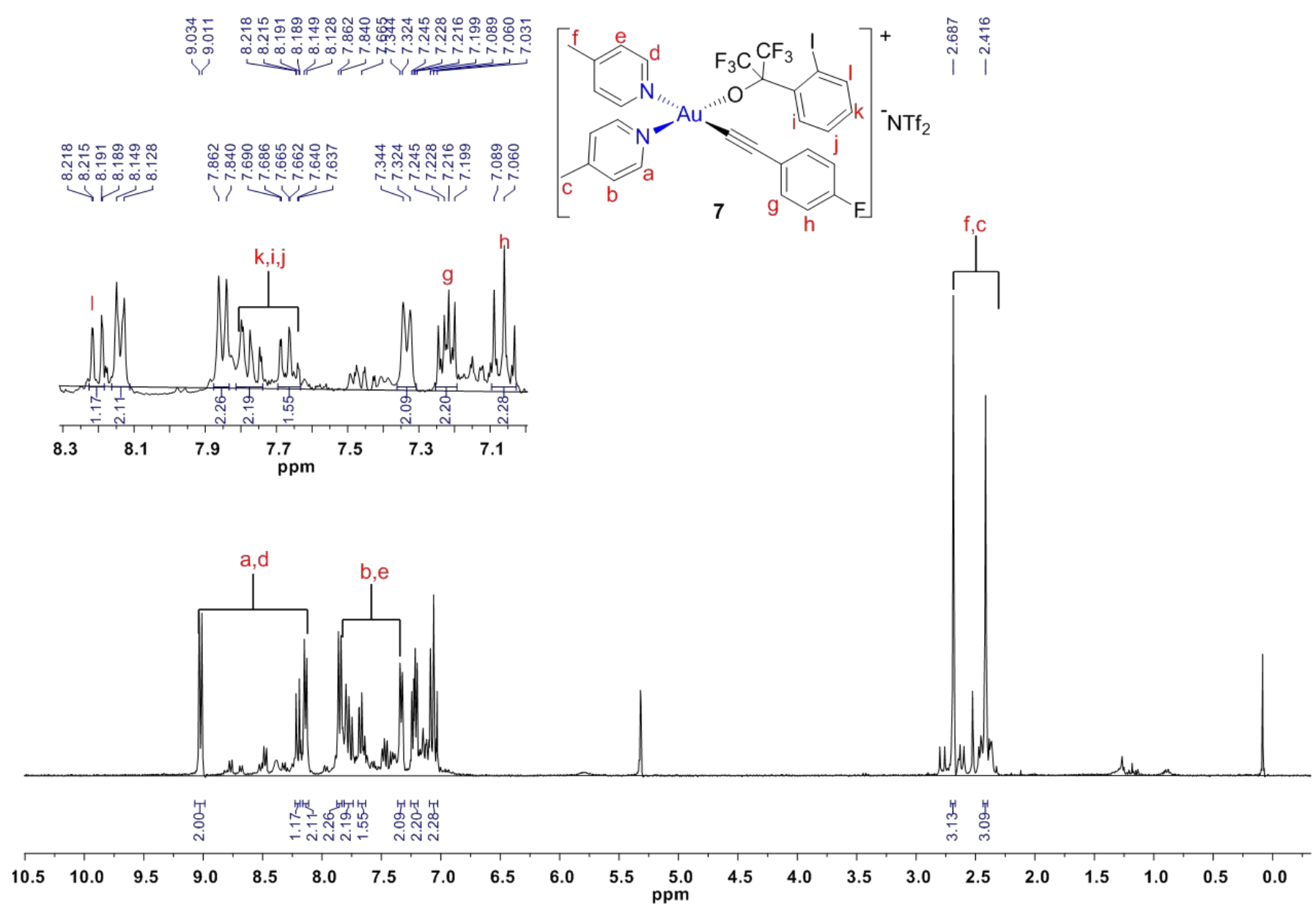

${ }^{19} \mathbf{F}$ NMR $\left(283 \mathrm{MHz}, \mathrm{CD}_{2} \mathrm{Cl}_{2}\right)$ spectrum of $7 \mathbf{a}$ 


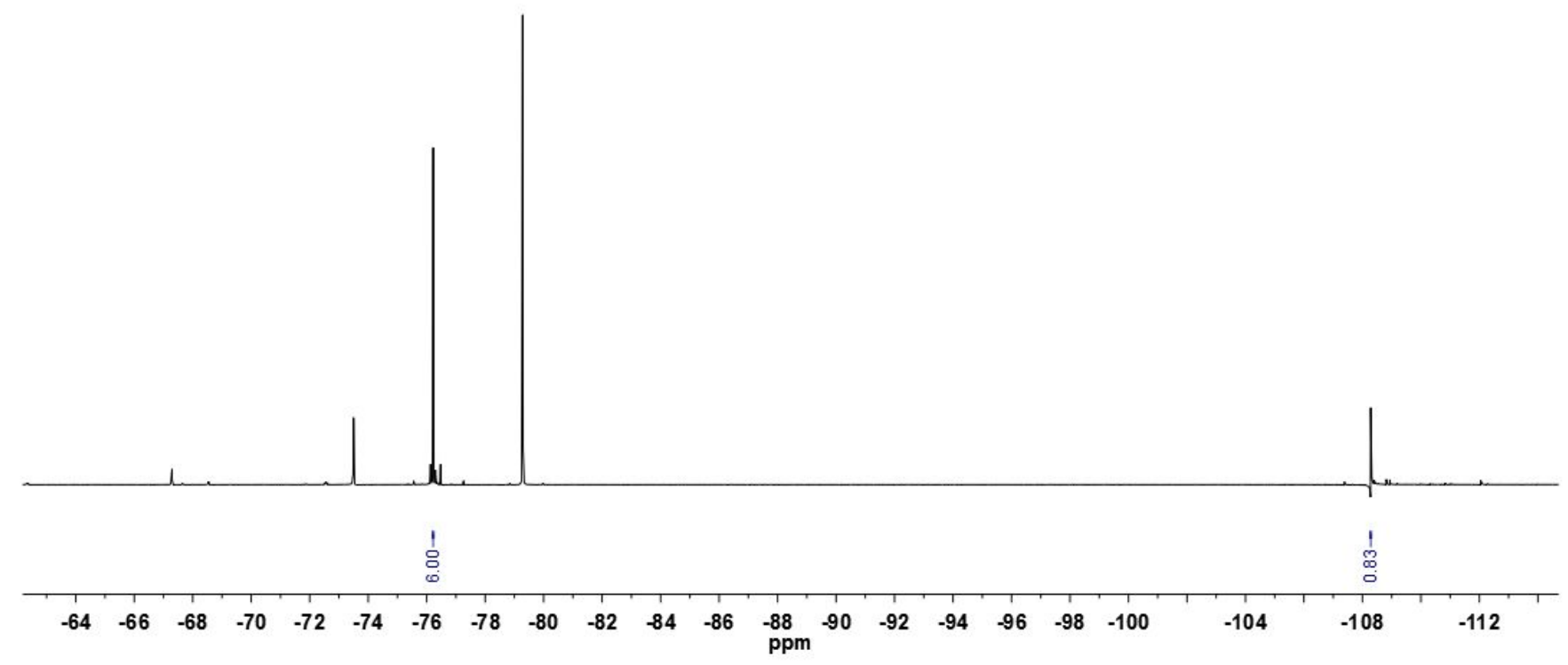

${ }^{1} \mathbf{H}$ NMR $\left(600 \mathrm{MHz}, \mathrm{CD}_{2} \mathrm{Cl}_{2}\right)$ spectrum of $7 \mathbf{c}$ 


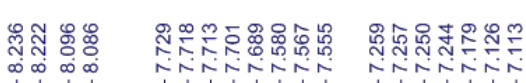
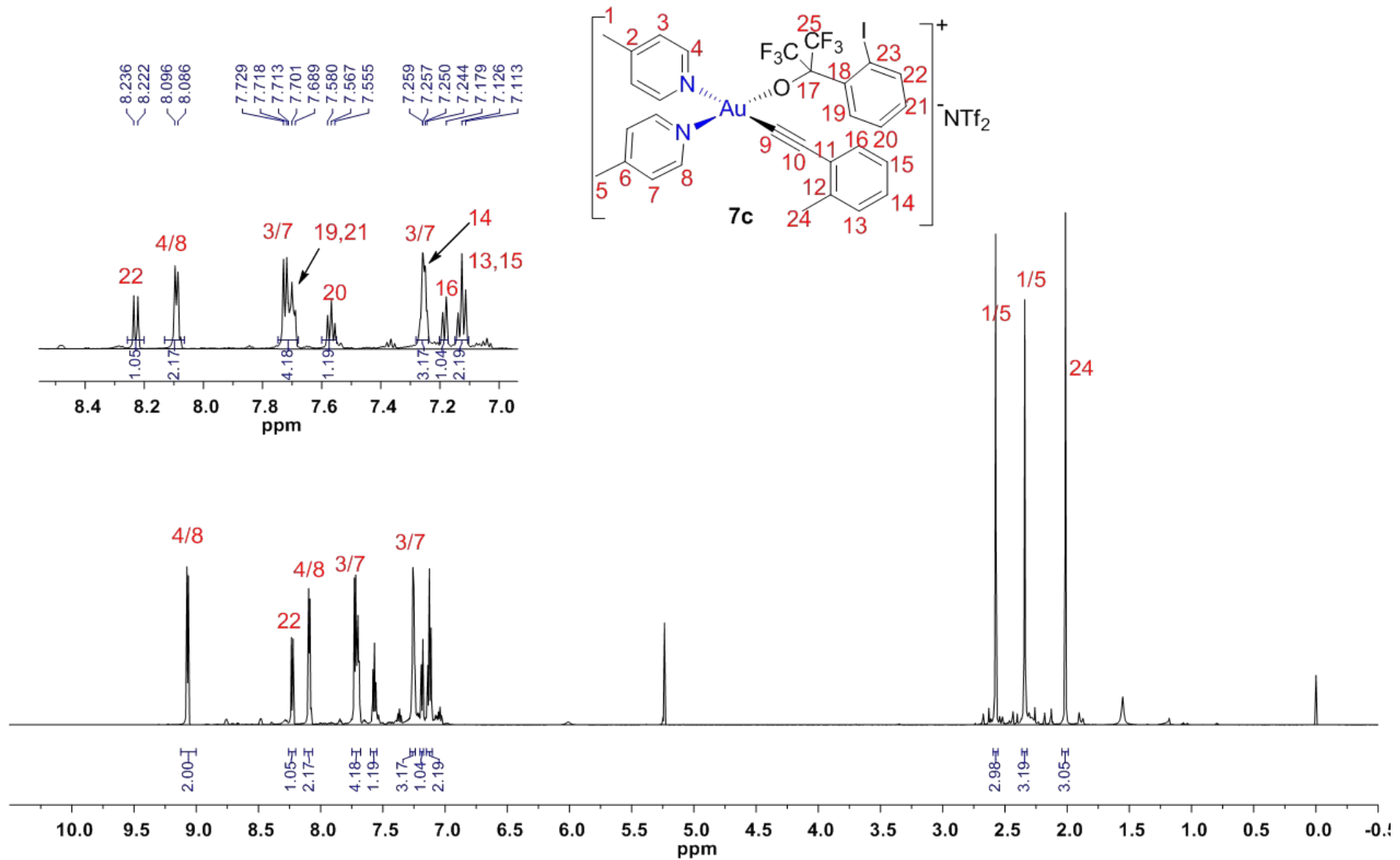

${ }^{13} \mathbf{C}$ NMR $\left(151 \mathrm{MHz}, \mathrm{CD}_{2} \mathrm{Cl}_{2}\right)$ spectrum of $7 \mathbf{c}$

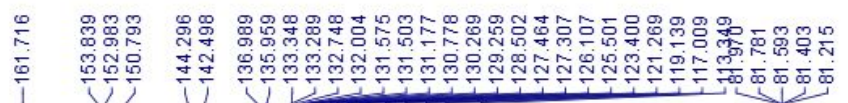

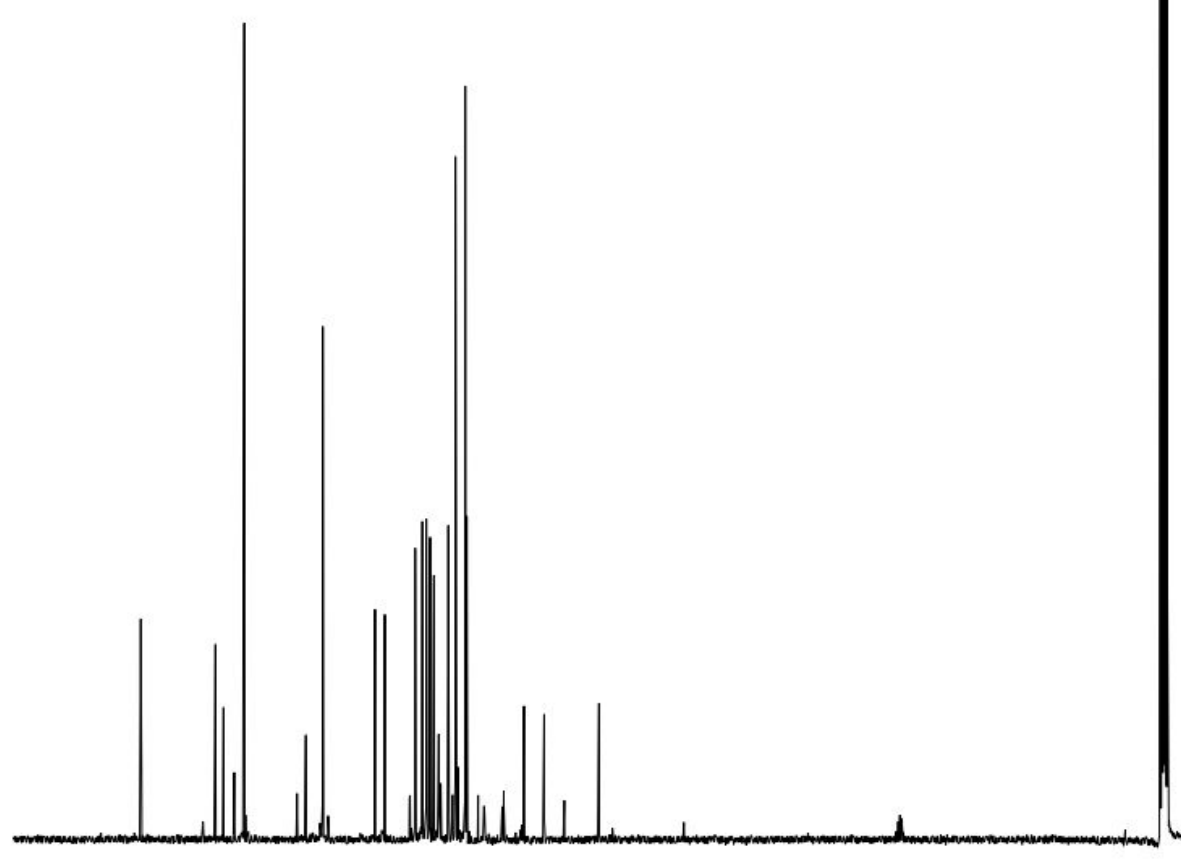

\section{\&્木}


${ }^{19}$ F NMR $\left(283 \mathrm{MHz}, \mathrm{CD}_{2} \mathrm{Cl}_{2}\right)$ spectrum of $7 \mathbf{c}$

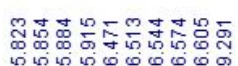

年

Li工is

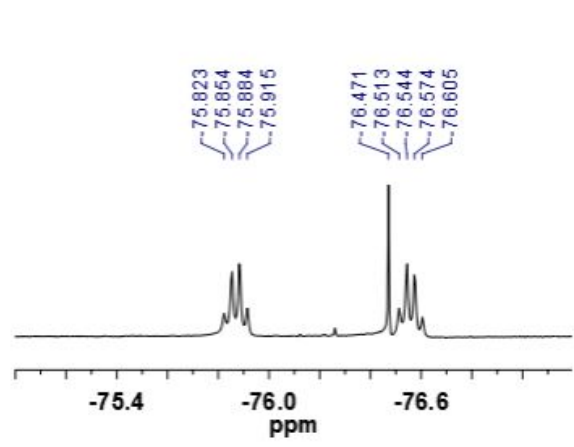

ppm

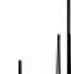

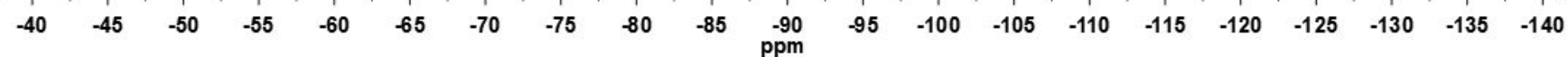

${ }^{1} \mathrm{H},{ }^{1} \mathrm{H}$-COSY spectrum of $7 \mathrm{c}$ 


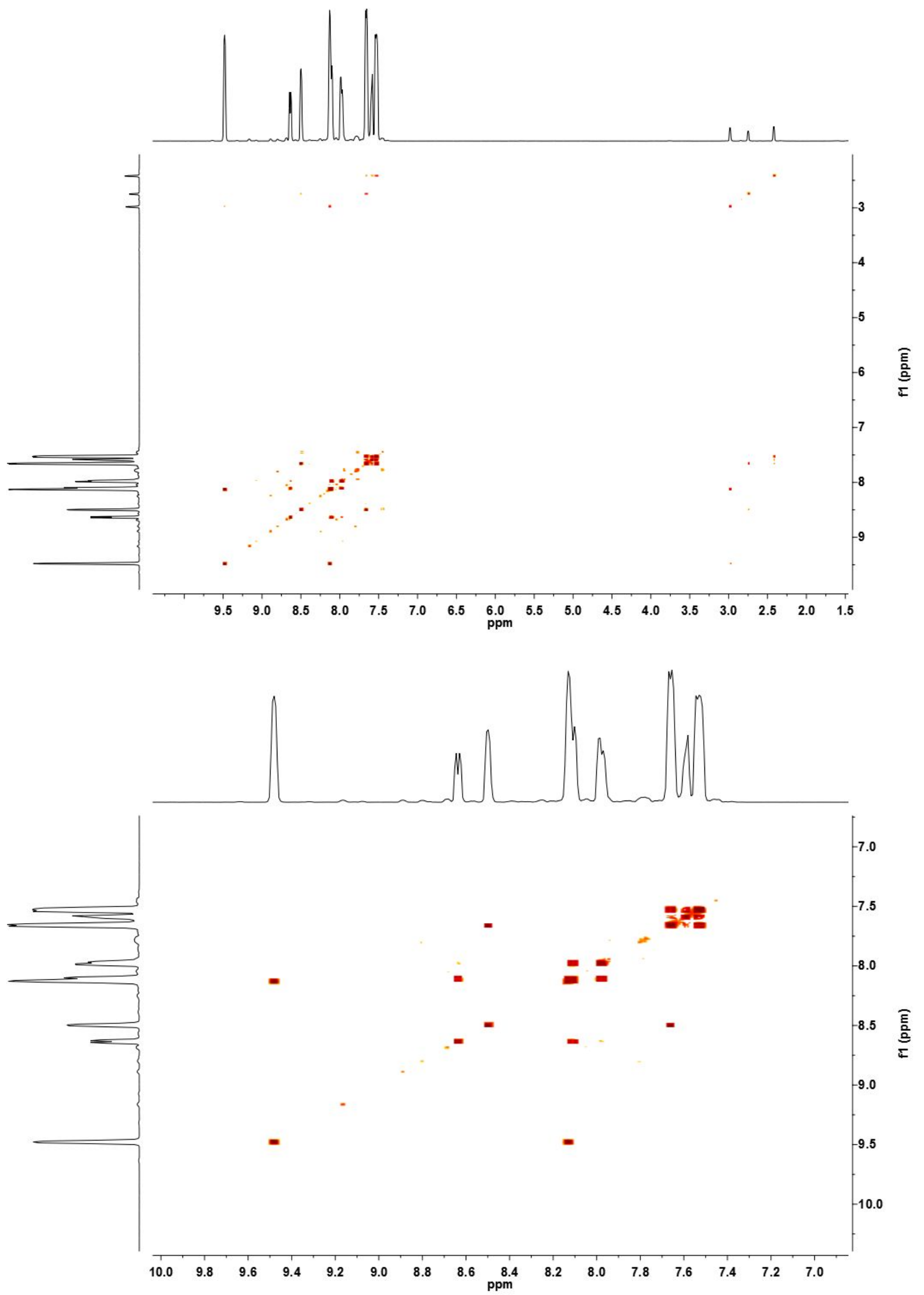


${ }^{1} \mathrm{H},{ }^{13} \mathrm{C}$-HSQC spectrum of $7 \mathrm{C}$

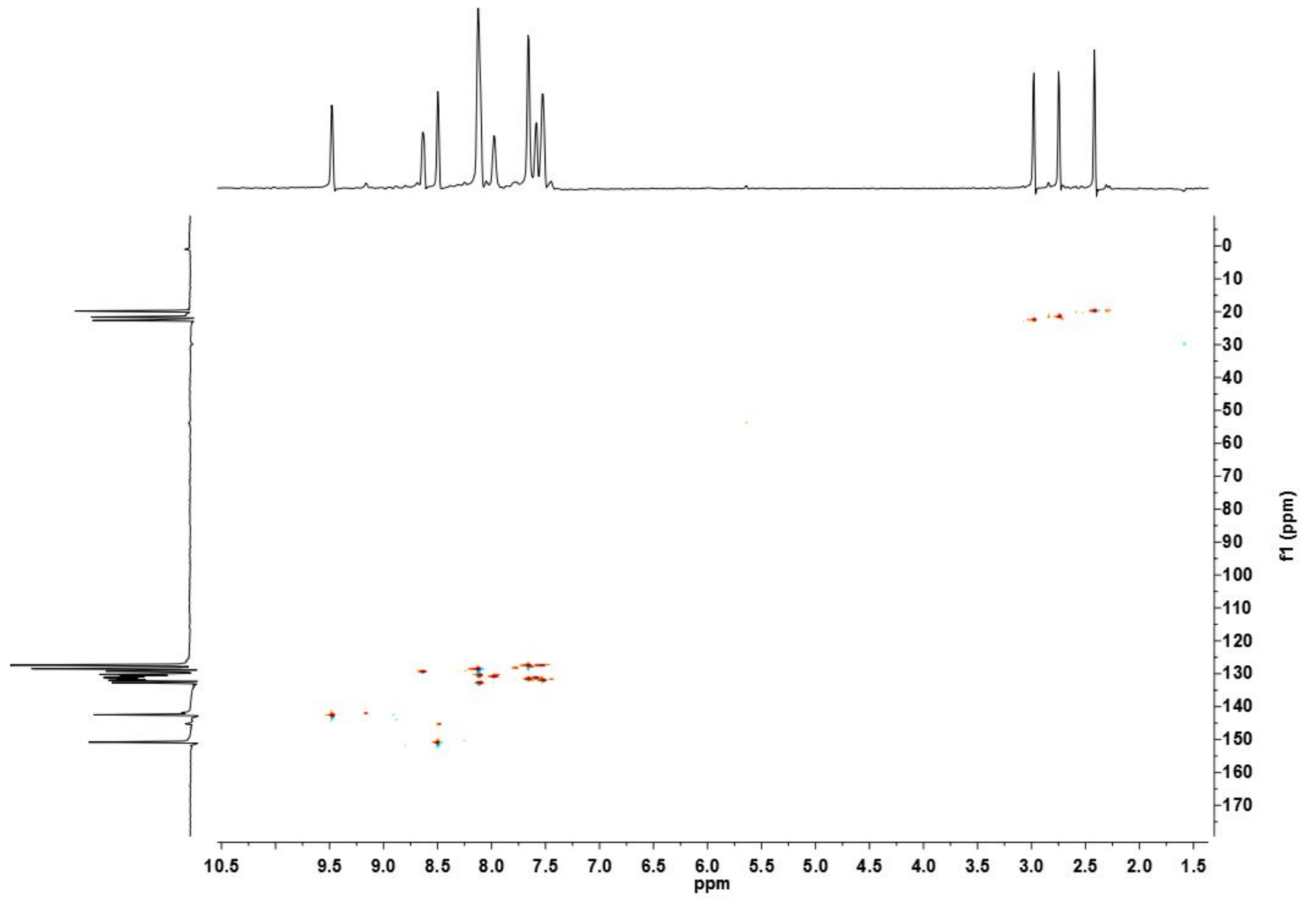




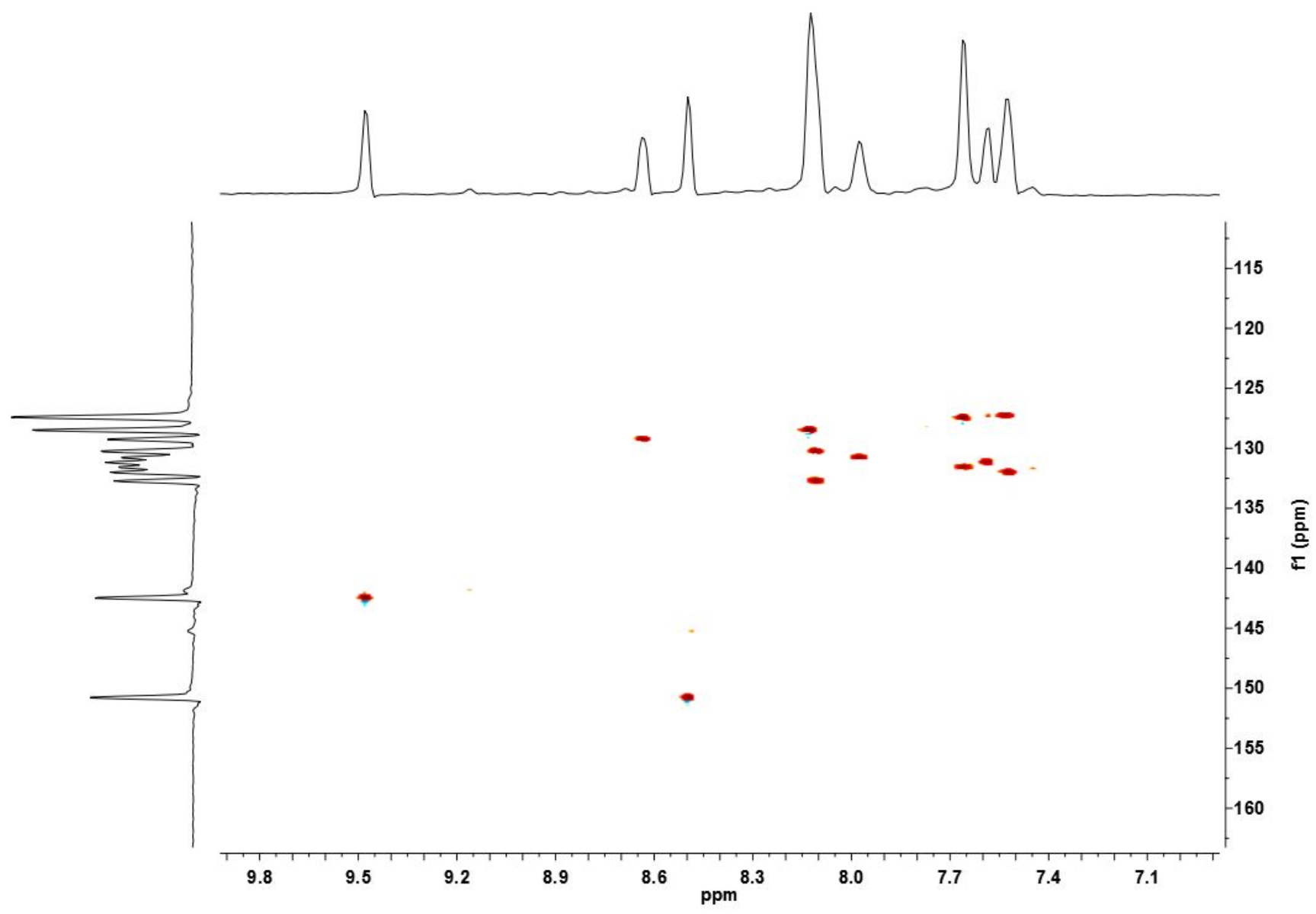

${ }^{1} \mathrm{H},{ }^{13} \mathrm{C}-\mathrm{HMBC}$ spectrum of $7 \mathrm{c}$ 


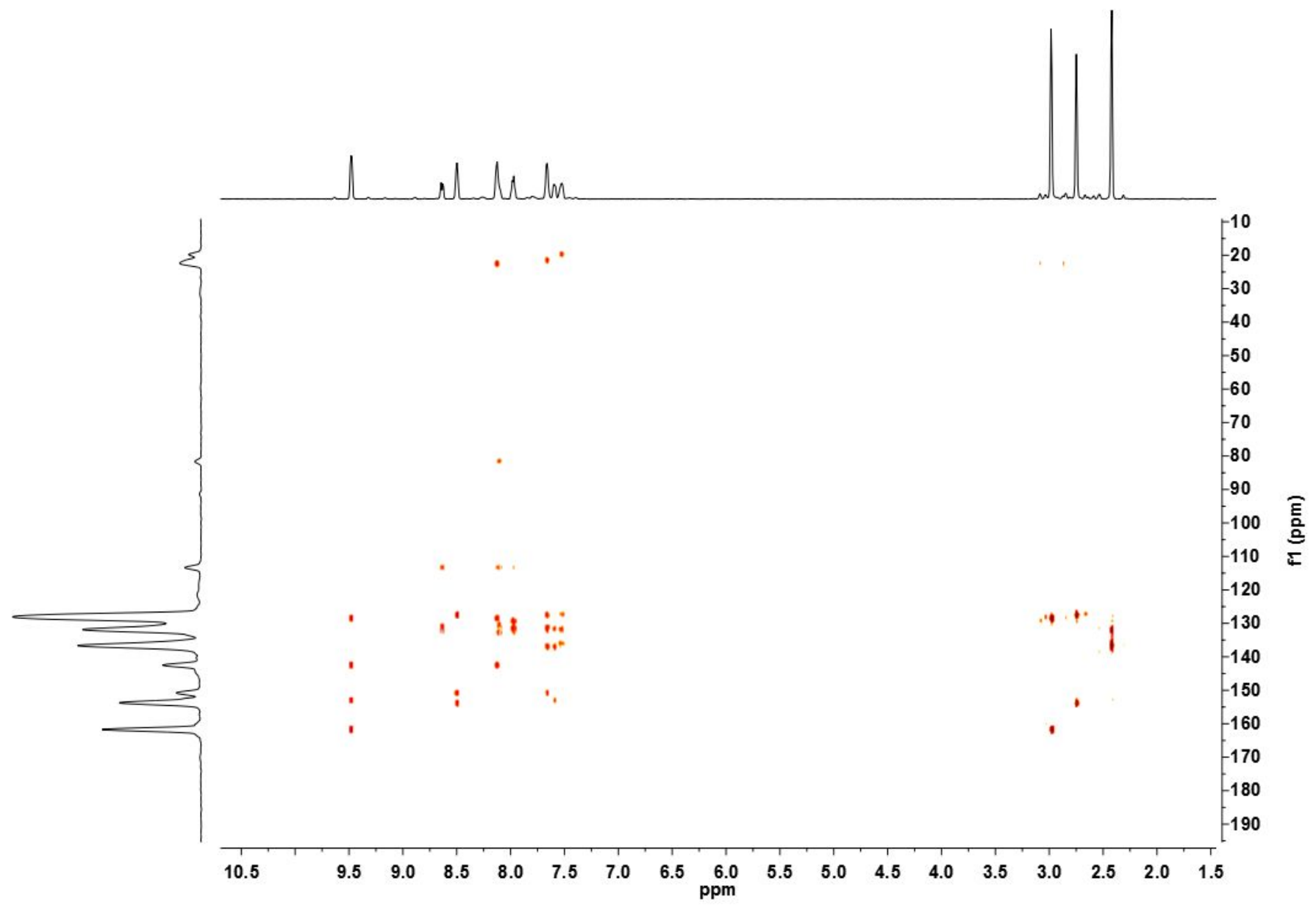




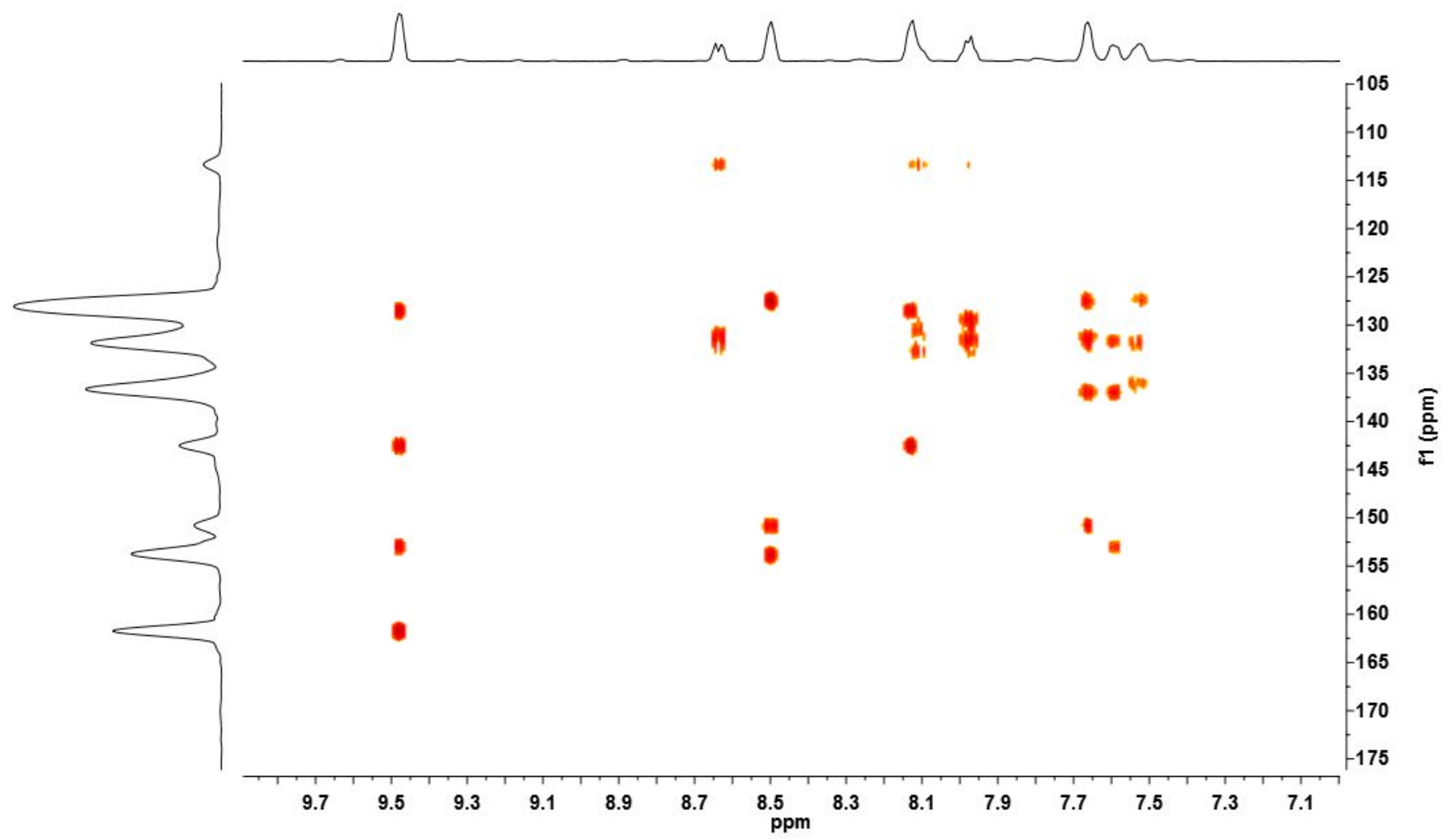

${ }^{1} \mathbf{H}$ NMR $\left(600 \mathrm{MHz}, \mathrm{CD}_{2} \mathrm{Cl}_{2}\right)$ spectrum of 7d 


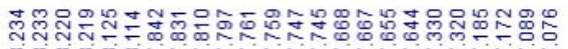
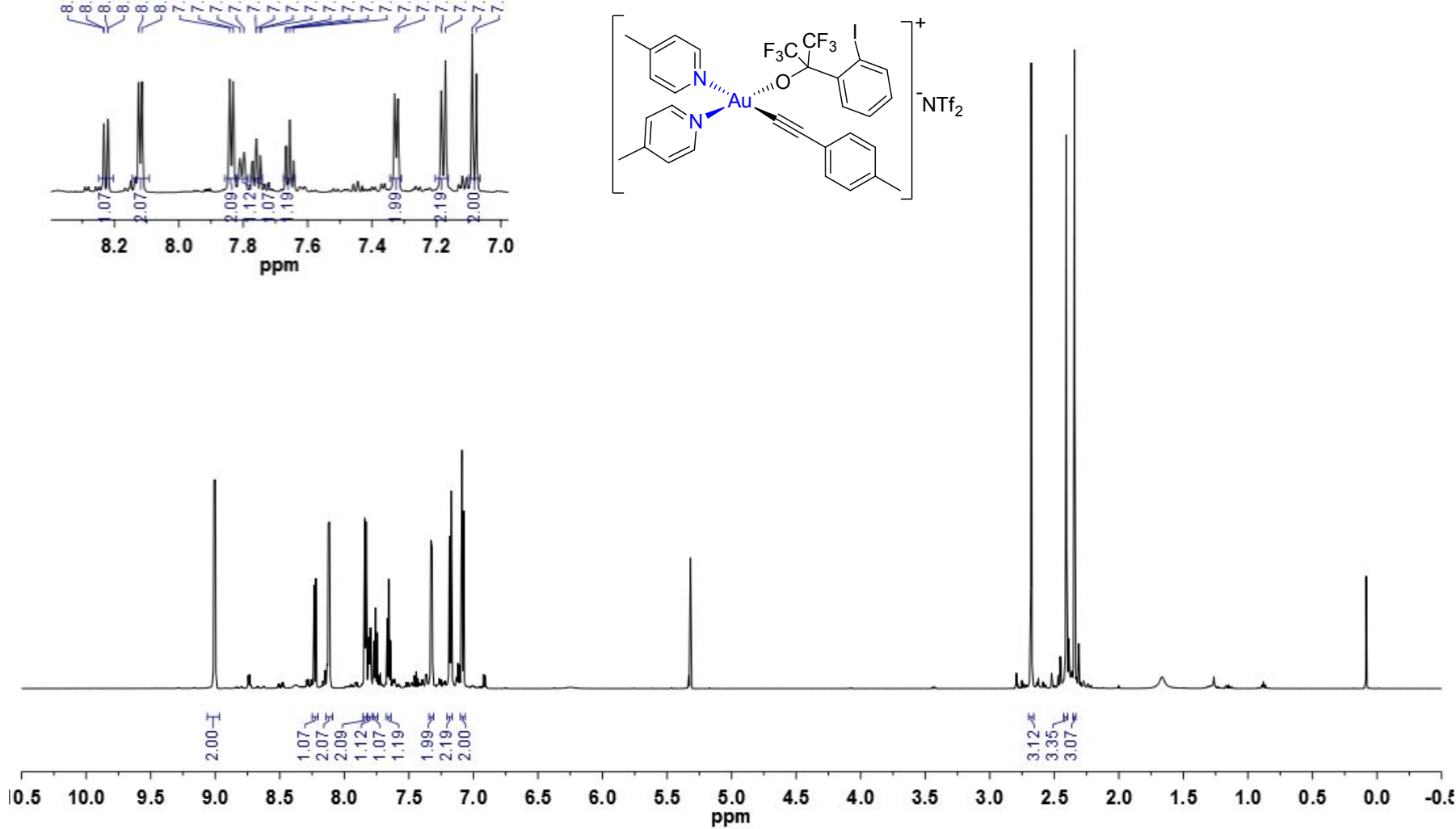

${ }^{13} \mathbf{C}$ NMR $\left(151 \mathrm{MHz}, \mathrm{CD}_{2} \mathrm{Cl}_{2}\right)$ spectrum of $\mathbf{7 d}$ 

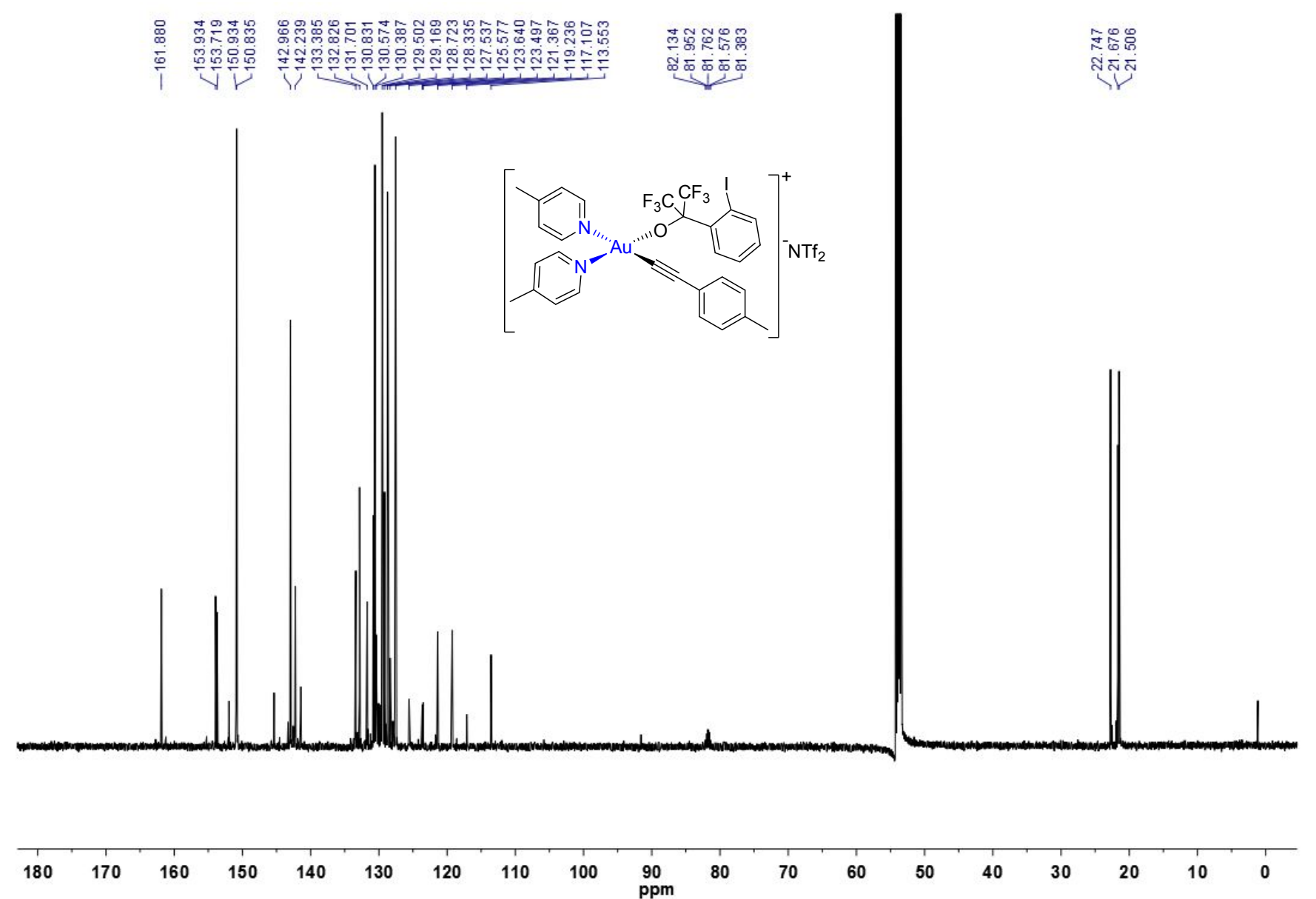

${ }^{19}$ F NMR $\left(283 \mathrm{MHz}, \mathrm{CD}_{2} \mathrm{Cl}_{2}\right)$ spectrum of 7d

\begin{tabular}{lllllllllllllllllllllllllllllllll}
\hline & -59 & -61 & -63 & -65 & -67 & -69 & -71 & -73 & -75 & -77 & -79 & -81 & -83 & -85 & -87 & -89 & -91 & -93 &
\end{tabular}


${ }^{1} \mathbf{H}$ NMR (301 MHz, $\left.\mathrm{CD}_{2} \mathrm{Cl}_{2}\right)$ spectrum of 7e

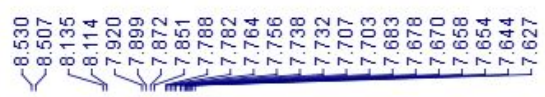<smiles></smiles>

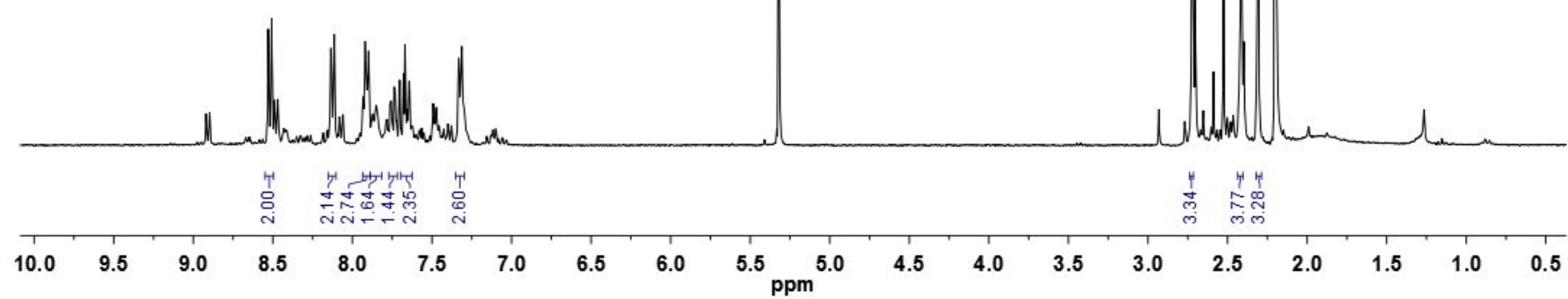

${ }^{19} \mathbf{F}$ NMR $\left(283 \mathrm{MHz}, \mathrm{CD}_{2} \mathrm{Cl}_{2}\right)$ spectrum of $7 \mathbf{e}$ 


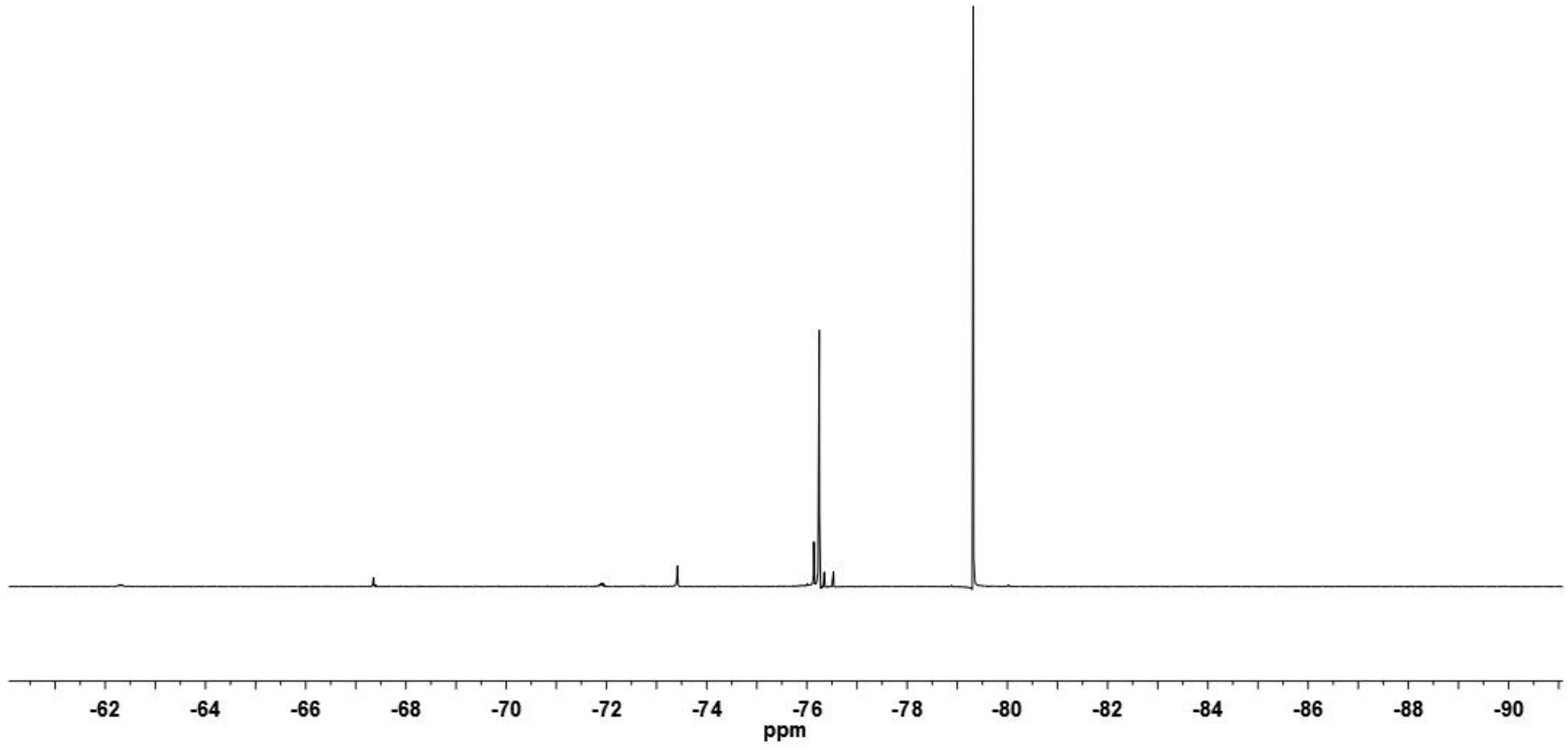

${ }^{1} \mathbf{H}$ NMR $\left(500 \mathrm{MHz}, \mathrm{CD}_{2} \mathrm{Cl}_{2}\right)$ spectrum of $7 \mathbf{f}$ 

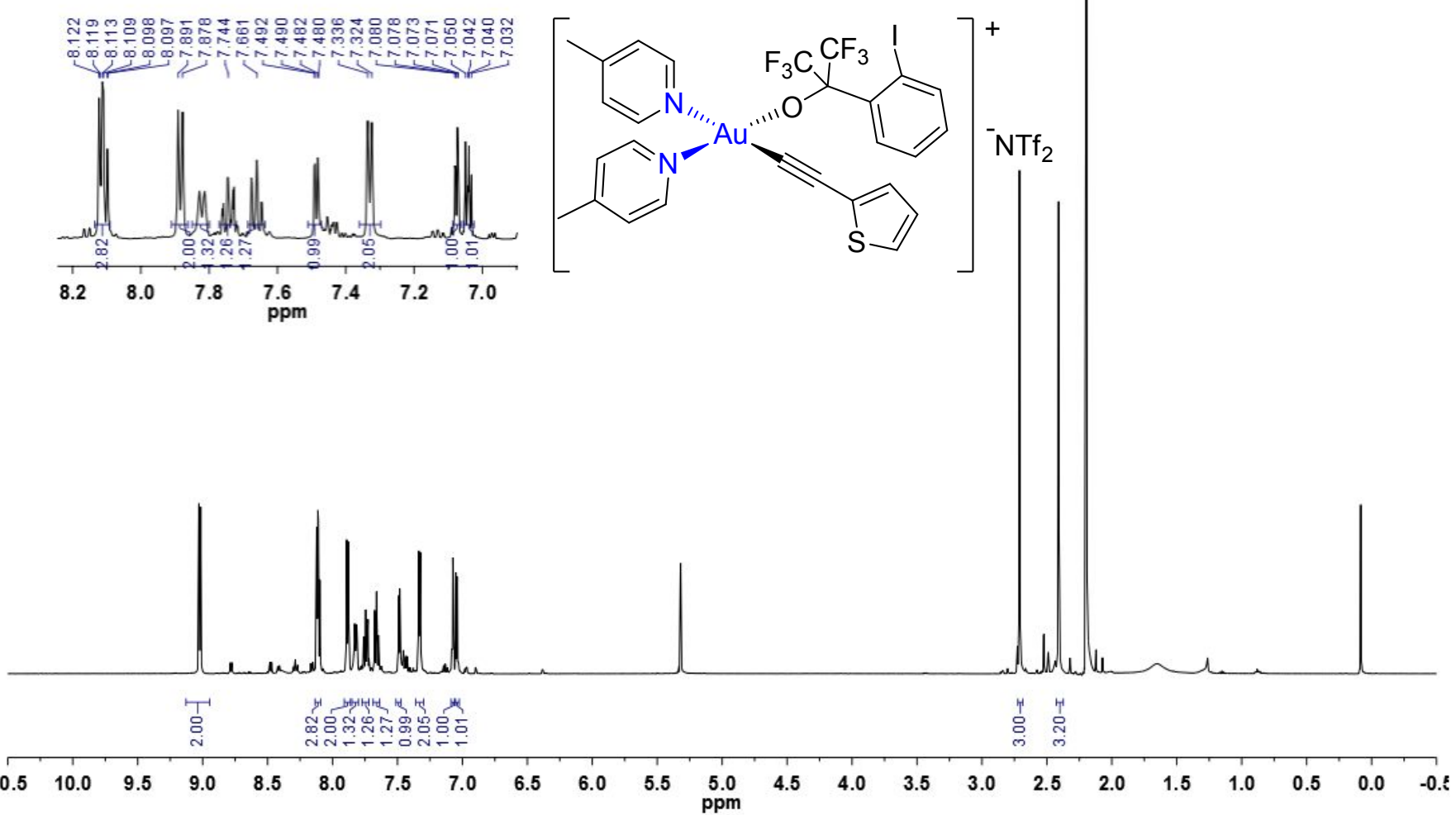

${ }^{19}$ F NMR (471 MHz, $\left.\mathrm{CD}_{2} \mathrm{Cl}_{2}\right)$ spectrum of $7 \mathbf{f}$

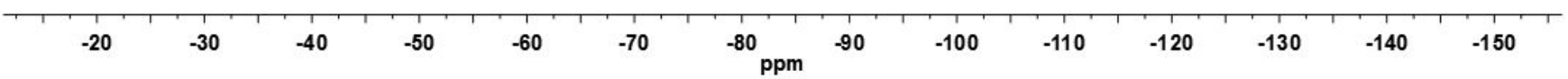


${ }^{1} \mathbf{H}$ NMR $\left(500 \mathrm{MHz}, \mathrm{CD}_{2} \mathrm{Cl}_{2}\right)$ spectrum of $7 \mathbf{g}$

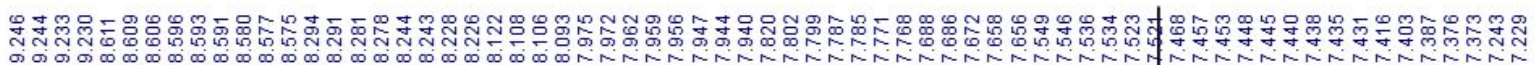

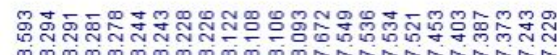

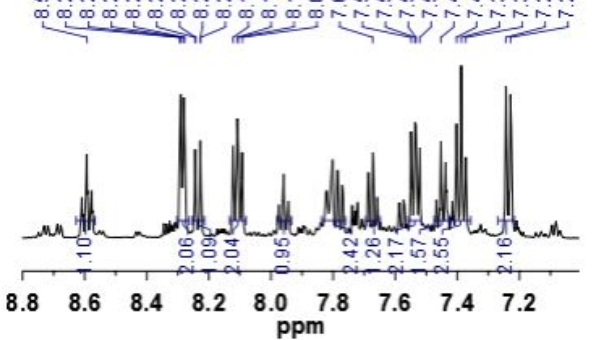<smiles></smiles>
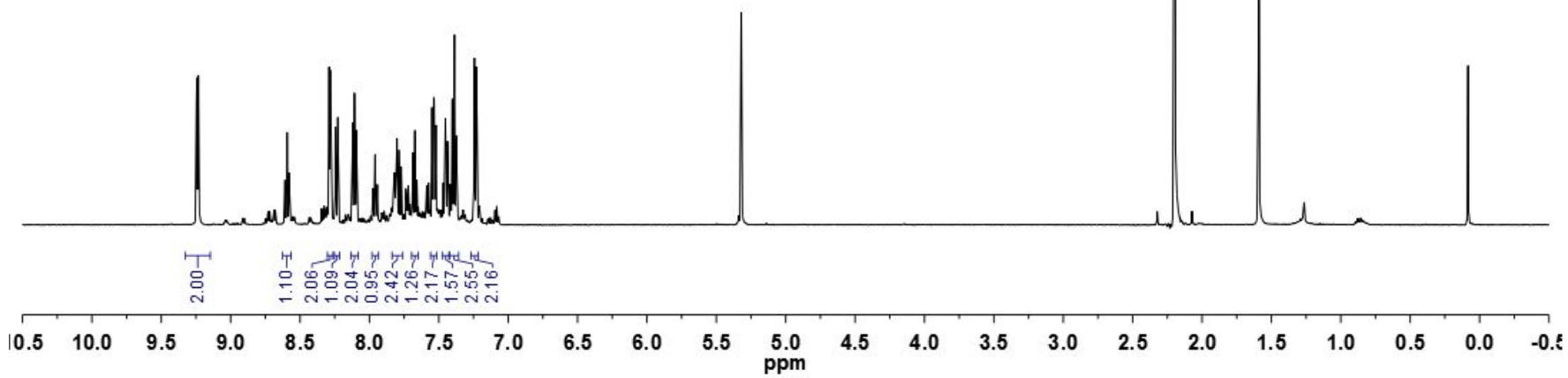

${ }^{19} \mathbf{F}$ NMR $\left(471 \mathrm{MHz}, \mathrm{CD}_{2} \mathrm{Cl}_{2}\right)$ spectrum of $7 \mathbf{g}$ 


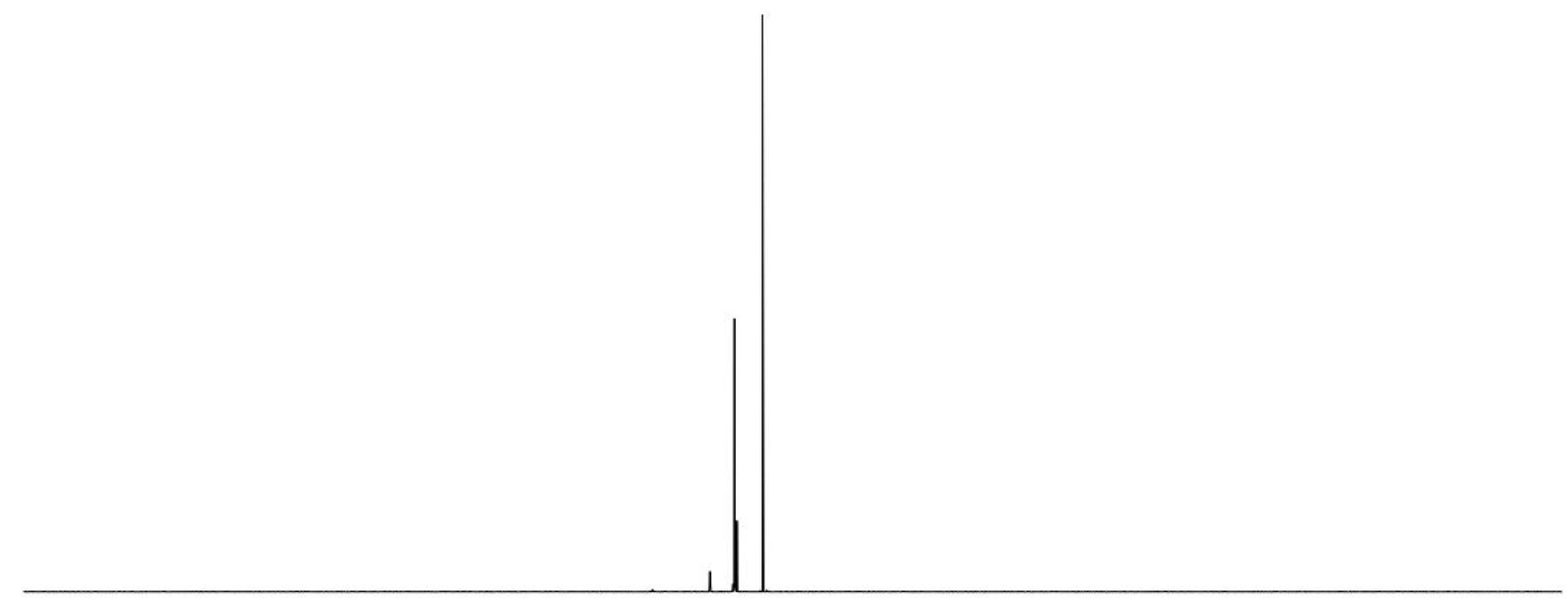

\begin{tabular}{|c|c|c|c|c|c|c|c|c|c|c|c|c|c|c|c|c|}
\hline 0 & -10 & -20 & -30 & -40 & -50 & -60 & -70 & $\stackrel{-80}{\mathrm{ppm}}$ & -90 & -100 & -110 & -120 & -130 & -140 & -150 & -160 \\
\hline
\end{tabular}

${ }^{\mathbf{1}} \mathbf{H}$ NMR (500 MHz, $\mathrm{CD}_{2} \mathrm{Cl}_{2}$ ) spectrum of $\mathbf{7 h}$

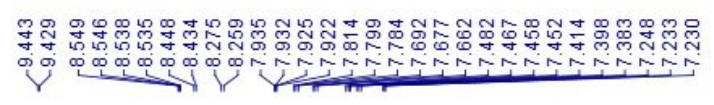

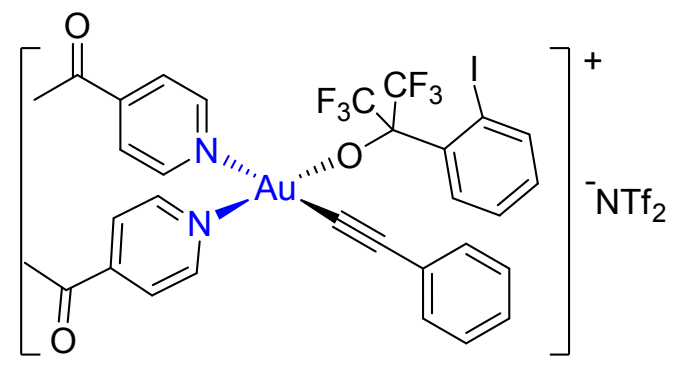

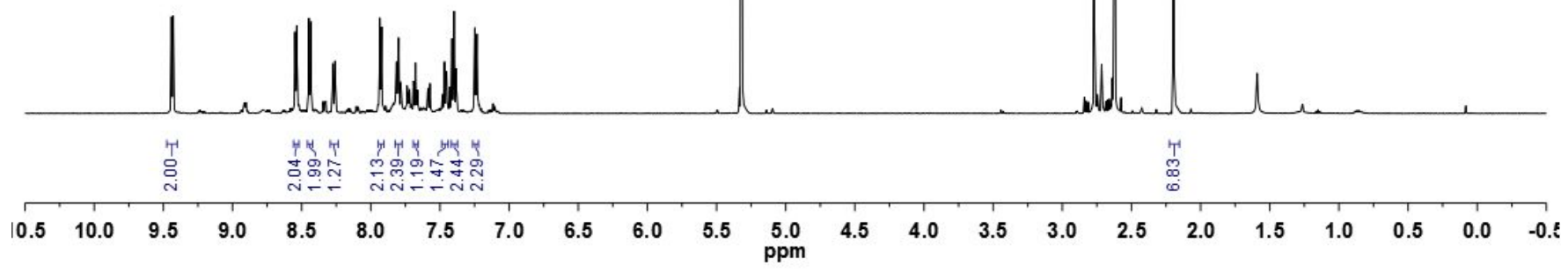


${ }^{19} \mathbf{F}$ NMR $\left(471 \mathrm{MHz}, \mathrm{CD}_{2} \mathrm{Cl}_{2}\right)$ spectrum of $7 \mathbf{h}$

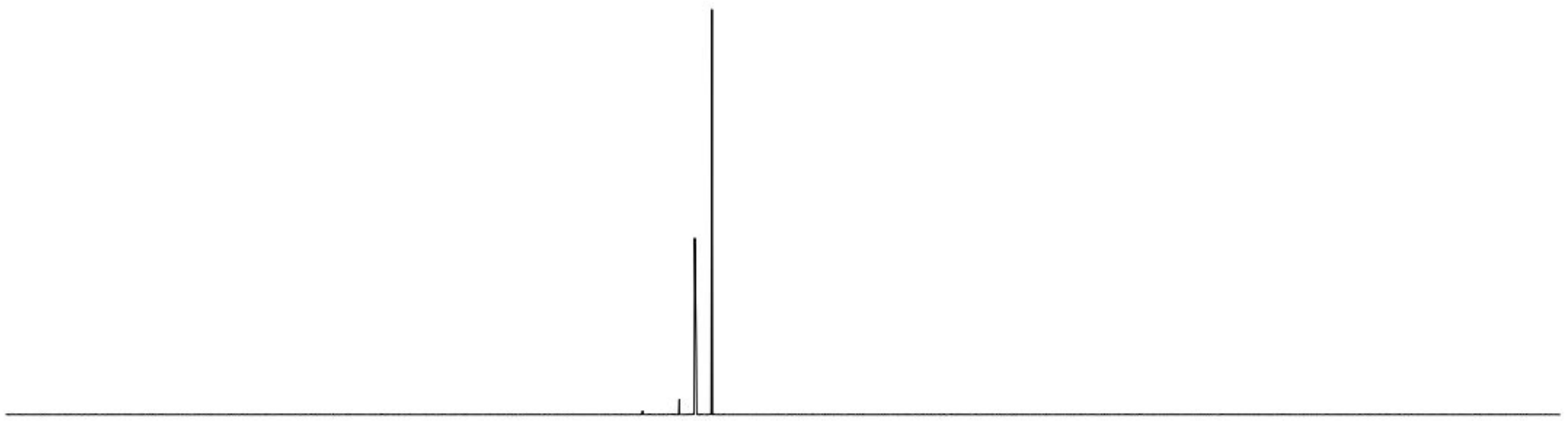

$\begin{array}{llllllllllllllllllll}40 & 30 & 20 & 10 & 0 & -10 & -20 & -30 & -40 & -50 & -60 & -70 & -80 & -90 & -110 & -130 & -150 & -170 & -190 & -210\end{array}$ 